\title{
Analytical periodic motions and bifurcations in a nonlinear rotor system
}

\author{
Jianzhe Huang • Albert C. J. Luo
}

Received: 15 October 2013 / Revised: 26 December 2013 / Accepted: 26 December 2013 / Published online: 9 February 2014

(C) Springer-Verlag Berlin Heidelberg 2014

\begin{abstract}
In this paper, analytical solutions for period- $m$ motions in a nonlinear rotor system are discussed. This rotor system with two-degrees of freedom is one of the simplest rotor dynamical systems, and periodic excitations are from the rotor eccentricity. The analytical expressions of periodic solutions are developed, and the corresponding stability and bifurcation analyses of period-m motions are carried out. Analytical bifurcation trees of period-1 motions to chaos are presented. The Hopf bifurcations of periodic motions cause not only the bifurcation tree but quasi-periodic motions. Displacement orbits of periodic motions in nonlinear rotor systems are used to illustrate motion complexity, and harmonic amplitude spectrums give harmonic effects on periodic motions of the nonlinear rotor. Coexisting periodic motions exist in the nonlinear rotor.
\end{abstract}

Keywords Period-m motions - Nonlinear rotor systems . Analytical bifurcation trees - Analytical dynamics - Stability and bifurcation - Harmonic balance method

\section{Introduction}

The rotor dynamics is about the vibration of rotating shaft with disks, and the shaft is supported by bearings with seals. In industrial application, flexible rotors are extensively used.

\section{J. Huang}

Department of Mechanical Engineering,

Washington University in St. Louis,

Saint Louis, MO 63130, USA

\section{A. C. J. Luo $(\bowtie)$}

Department of Mechanical and Industrial Engineering,

Southern Illinois University Edwardsville,

Edwardsville, IL 62026-1805, USA

e-mail: aluo@siue.edu
The high performance machines always operate over the first critical speed. In 1883, Gustav Delaval for the first time manufactured a gas turbine which can operate over the first critical rotation speed. In 1919, Jeffcott [1] first developed equations of motion for linear rotor dynamics. For such a linear rotor system, it can be easily analyzed. However, the results may not be adequate for flexible rotors with high operation speed. Thus one considered the bearing clearance, squeezing film dampers, seals and fluid dynamics effects in flexible rotor systems. In 1974, Begg [2] investigated stability of a friction-induced rotor whirl motions. In 1982, Childs [3] applied a perturbation method to investigate subharmonic responses of a rotor with a small nonlinearity. In 1987, Choi and Noah [4] use the harmonic balance method (HBM) and fast Fourier transformation (FFT) to study subharmonic and supperharmonic responses in a rotor with a bearing clearance. In 1988, Ehrich [5] numerically investigated higher order suhharmonic responses in such a rotor system under a high operation speed. In addition, Day [6] used the multiplescale method to show the aperiodic motion. In 1990, Kim and Noah [7] used the harmonic balance method to discuss the bifurcation of periodic motions in a modified Jeffcott rotor with bearing clearings. In 1995, Choi and Noah [8] still used the harmonic balance method to investigate mode-locking motion and chaos in such a Jeffcott rotor. The quasi-periodic motions and stability for such a modified Jeffcott rotor was also presented through the harmonic balance method in Kim and Noah [9]. In 1998, Chu and Zhang [10] used the harmonic balance method to determine periodic motions and numerically show the bifurcation scenarios. In fact, the modified Jeffcott rotor is discontinuous. Thus, the harmonic balance method may not be an adequate method for periodic motions in such a modified rotor with discontinuity, which can be only as a rough prediction. In 2001, Jiang and Ulbrich [11] investigated stability of sliding whirl in a nonlinear Jeffcott rotor. 
In 2012, Luo [12] developed a generalized harmonic balance method to get the approximate analytical solutions of periodic motions and chaos in nonlinear dynamical systems. This method used the finite Fourier series to express periodic motions, and the coefficients are time-varying. With the principle of virtual work, a dynamical system of coefficients is obtained from which the steady-state solution is achieved, and the corresponding stability and bifurcation are completed. Luo and Huang [14] used the generalized harmonic balance method to obtain approximate analytical solutions of periodic motions in the Duffing oscillator, and the analytical bifurcation trees of periodic motions to chaos are obtained. Luo and Huang [15] employed the generalized harmonic balance method with finite terms to obtain the analytical solution of period-1 motion of the Duffing oscillator with a twin-well potential. Luo and Huang [16] used a generalized harmonic balance method to find analytical solutions of period- $m$ motions in such a Duffing oscillator. The analytical bifurcation trees of periodic motions in the Duffing oscillator to chaos are obtained (also see, Luo and Huang [17-19]). Such analytical bifurcation trees show the connection from periodic solution to chaos analytically. To better understand nonlinear behaviors in nonlinear dynamical systems, the analytical solutions for the bifurcation trees from period-1 motion to chaos in a periodically forced oscillator with quadratic nonlinearity were presented in Luo and $\mathrm{Yu}$ [20-22], and period-m motions in the periodically forced, van der Pol equation was presented in Luo and Laken [23]. As aforementioned, the periodic solutions for one-degree of freedom nonlinear systems were investigated. Herein, the new method will be used to investigate nonlinear dynamics of rotor dynamical systems.

In this paper, analytical solutions for period- $m$ motions in a nonlinear rotor system will be developed, and the stability and bifurcation analyses of period-m motions will be carried out. Analytical bifurcation trees of period- 1 motions to chaos will be presented. Illustrations of periodic and quasi-periodic motions will be completed to show nonlinear behaviors in the nonlinear rotor system.

\section{Analytical solutions}

Consider a nonlinear rotor system

$$
\begin{aligned}
& \ddot{x}+\delta \dot{x}+(1+\gamma)\left[\alpha x+\beta x\left(x^{2}+y^{2}\right)\right]=e \Omega^{2} \cos \Omega t, \\
& \ddot{y}+\delta \dot{y}+\alpha y+\beta y\left(x^{2}+y^{2}\right)=e \Omega^{2} \sin \Omega t
\end{aligned}
$$

where $\delta$ is the linear damping coefficient. $\alpha$ and $\beta$ are linear and cubic spring coefficients, respectively. $e$ and $\Omega$ are the eccentric distance and rotational speed of the rotor, respectively. $\gamma$ is an asymmetric coefficient. The standard form of
Eq. (1) is

$\ddot{x}+f_{1}(x, y, \dot{x}, \dot{y}, t)=0, \ddot{y}+f_{2}(x, y, \dot{x}, \dot{y}, t)=0$

where

$$
\begin{aligned}
f_{1}(x, y, \dot{x}, \dot{y}, t)= & \delta \dot{x}+(1+\gamma)\left[\alpha x+\beta x\left(x^{2}+y^{2}\right)\right] \\
& -e \Omega^{2} \cos \Omega t \\
f_{2}(x, y, \dot{x}, \dot{y}, t)= & \delta \dot{y}+\alpha y+\beta y\left(x^{2}+y^{2}\right)-e \Omega^{2} \sin \Omega t .
\end{aligned}
$$

In Luo [13], consider the analytical solution of period- $m$ motion as

$$
\begin{aligned}
x^{*}(t)= & a_{10}^{(m)}(t)+\sum_{k=1}^{N} b_{1 k / m}(t) \cos \left(\frac{k}{m} \Omega t\right) \\
& +c_{1 k / m}(t) \sin \left(\frac{k}{m} \Omega t\right), \\
y^{*}(t)= & a_{20}^{(m)}(t)+\sum_{k=1}^{N} b_{2 k / m}(t) \cos \left(\frac{k}{m} \Omega t\right) \\
& +c_{2 k / m}(t) \sin \left(\frac{k}{m} \Omega t\right) .
\end{aligned}
$$

Then the first and second order derivatives of $x^{*}(t)$ and $y^{*}(t)$ are

$$
\begin{aligned}
\dot{x}^{*}(t)= & \dot{a}_{10}^{(m)}+\sum_{k=1}^{N}\left(\dot{b}_{1 k / m}+\frac{k \Omega}{m} c_{1 k / m}\right) \cos \left(\frac{k \Omega t}{m}\right) \\
& +\left(\dot{c}_{1 k / m}-\frac{k \Omega}{m} b_{1 k / m}\right) \sin \left(\frac{k \Omega t}{m}\right) \\
\dot{y}^{*}(t)= & \dot{a}_{20}^{(m)}+\sum_{k=1}^{N}\left(\dot{b}_{2 k / m}+\frac{k \Omega}{m} c_{2 k / m}\right) \cos \left(\frac{k \Omega t}{m}\right) \\
& +\left(\dot{c}_{2 k / m}-\frac{k \Omega}{m} b_{2 k / m}\right) \sin \left(\frac{k \Omega t}{m}\right)
\end{aligned}
$$

$$
\begin{aligned}
\ddot{x}^{*}(t)= & \ddot{a}_{10}^{(m)}+\sum_{k=1}^{N}\left[\ddot{b}_{1 k / m}+2 \frac{k \Omega}{m} \dot{c}_{1 k / m}-\left(\frac{k \Omega}{m}\right)^{2} b_{1 k / m}\right] \\
& \times \cos \left(\frac{k \Omega}{m} t\right)+\left[\ddot{c}_{1 k / m}-2 \frac{k \Omega}{m} \dot{b}_{1 k / m}-\left(\frac{k \Omega}{m}\right)^{2} c_{1 k / m}\right] \\
& \times \sin \left(\frac{k \Omega}{m} t\right),
\end{aligned}
$$

$$
\begin{aligned}
\ddot{y}^{*}(t)= & \ddot{a}_{20}^{(m)}+\sum_{k=1}^{N}\left[\ddot{b}_{2 k / m}+2 \frac{k \Omega}{m} \dot{c}_{2 k / m}\right. \\
& \left.-\left(\frac{k \Omega}{m}\right)^{2} b_{2 k / m}\right] \cos \left(\frac{k \Omega}{m} t\right)
\end{aligned}
$$




$$
\begin{aligned}
& +\left[\ddot{c}_{2 k / m}-2 \frac{k \Omega}{m} \dot{b}_{2 k / m}-\left(\frac{k \Omega}{m}\right)^{2} c_{2 k / m}\right] \\
& \times \sin \left(\frac{k \Omega}{m} t\right) .
\end{aligned}
$$

Define

$$
\begin{aligned}
\mathbf{a}_{0}^{(m)} & =\left(a_{10}^{(m)}, a_{20}^{(m)}\right)^{\mathrm{T}}, \\
\mathbf{b}^{(m)} & =\left(b_{11 / m}, \ldots, b_{1 N / m} ; b_{21 / m}, \ldots, b_{2 N / m}\right)^{\mathrm{T}} \\
& =\left(\mathbf{b}_{1}^{(m)}, \mathbf{b}_{2}^{(m)}\right)^{\mathrm{T}}, \\
\mathbf{c}^{(m)} & =\left(c_{11 / m}, \ldots, c_{1 N / m} ; c_{21 / m}, \ldots, c_{2 N / m}\right)^{\mathrm{T}} \\
& =\left(\mathbf{c}_{1}^{(m)} ; \mathbf{c}_{2}^{(m)}\right)^{\mathrm{T}} .
\end{aligned}
$$

Substitution of Eqs. (4)-(6) into Eq. (3) and averaging all terms of $\cos (k \Omega t / m)$ and $\sin (k \Omega t / m)$ term gives

$$
\begin{aligned}
& \ddot{a}_{10}^{(m)}+F_{10}^{(m)}\left(\mathbf{a}_{0}^{(m)}, \mathbf{b}^{(m)}, \mathbf{c}^{(m)}, \dot{\mathbf{a}}_{0}^{(m)}, \dot{\mathbf{b}}^{(m)}, \dot{\mathbf{c}}^{(m)}\right)=0 \\
& \ddot{b}_{1 k / m}+2 \frac{k \Omega}{m} \dot{c}_{1 k / m}-\left(\frac{k \Omega}{m}\right)^{2} b_{1 k / m} \\
& \quad+F_{1 k / m}^{(c)}\left(\mathbf{a}_{0}^{(m)}, \mathbf{b}^{(m)}, \mathbf{c}^{(m)}, \dot{\mathbf{a}}_{0}^{(m)}, \dot{\mathbf{b}}^{(m)}, \dot{\mathbf{c}}^{(m)}\right)=0 \\
& \ddot{c}_{1 k / m}-2 \frac{k \Omega}{m} \dot{b}_{1 k / m}-\left(\frac{k \Omega}{m}\right)^{2} c_{1 k / m} \\
& \quad+F_{1 k / m}^{(s)}\left(\mathbf{a}_{0}^{(m)}, \mathbf{b}^{(m)}, \mathbf{c}^{(m)}, \dot{\mathbf{a}}_{0}^{(m)}, \dot{\mathbf{b}}^{(m)}, \dot{\mathbf{c}}^{(m)}\right)=0 \\
& \ddot{a}_{20}^{(m)}+F_{20}^{(m)}\left(\mathbf{a}_{0}^{(m)}, \mathbf{b}^{(m)}, \mathbf{c}^{(m)}, \dot{\mathbf{a}}_{0}^{(m)}, \dot{\mathbf{b}}^{(m)}, \dot{\mathbf{c}}^{(m)}\right)=0 \\
& \ddot{b}_{2 k / m}+2 \frac{k \Omega}{m} \dot{c}_{2 k / m}-\left(\frac{k \Omega}{m}\right)^{2} b_{2 k / m} \\
& \quad+F_{2 k / m}^{(c)}\left(\mathbf{a}_{0}^{(m)}, \mathbf{b}^{(m)}, \mathbf{c}^{(m)}, \dot{\mathbf{a}}_{0}^{(m)}, \dot{\mathbf{b}}^{(m)}, \dot{\mathbf{c}}^{(m)}\right)=0 \\
& \ddot{c}_{2 k / m}-2 \frac{k \Omega}{m} \dot{b}_{2 k / m}-\left(\frac{k \Omega}{m}\right)^{2} c_{2 k / m} \\
& \quad+F_{2 k / m}^{(s)}\left(\mathbf{a}_{0}^{(m)}, \mathbf{b}^{(m)}, \mathbf{c}^{(m)}, \dot{\mathbf{a}}_{0}^{(m)}, \dot{\mathbf{b}}^{(m)}, \dot{\mathbf{c}}^{(m)}\right)=0 .
\end{aligned}
$$

where

$$
\begin{aligned}
& F_{10}^{(m)}\left(\mathbf{a}_{0}^{(m)}, \mathbf{b}^{(m)}, \mathbf{c}^{(m)}, \dot{\mathbf{a}}_{0}^{(m)}, \dot{\mathbf{b}}^{(m)}, \dot{\mathbf{c}}^{(m)}\right) \\
& =\frac{1}{m T} \int_{0}^{m T} F_{1}\left(\mathbf{x}^{(m) *}, \mathbf{y}^{(m) *}, t\right) d t \\
& \quad=\delta \dot{a}_{10}^{(m)}+(1+\gamma)\left(\alpha a_{10}^{(m)}+\beta f_{10}^{(m)}\right), \\
& F_{1 k}^{(c)}\left(\mathbf{a}_{0}^{(m)}, \mathbf{b}^{(m)}, \mathbf{c}^{(m)}, \dot{\mathbf{a}}_{0}^{(m)}, \dot{\mathbf{b}}^{(m)}, \dot{\mathbf{c}}^{(m)}\right) \\
& \quad=\frac{2}{m T} \int_{0}^{m T} F_{1}\left(\mathbf{x}^{(m) *}, \mathbf{y}^{(m) *}, t\right) \cos \left(\frac{k}{m} \Omega t\right) d t
\end{aligned}
$$

$$
\begin{aligned}
= & \delta\left(\dot{b}_{1 k / m}+\frac{k \Omega}{m} c_{1 k / m}\right)+(1+\gamma)\left(\alpha b_{1 k / m}\right. \\
& \left.+\beta f_{1 k / m}^{(c)}\right)-e \Omega^{2} \delta_{k}^{m},
\end{aligned}
$$$$
=\delta\left(\dot{c}_{1 k / m}-\frac{k \Omega}{m} b_{1 k / m}\right)+(1+\gamma)\left(\alpha c_{1 k / m}+\beta f_{1 k / m}^{(s)}\right) .
$$$$
F_{20}^{(m)}\left(\mathbf{a}_{0}^{(m)}, \mathbf{b}^{(m)}, \mathbf{c}^{(m)}, \dot{\mathbf{a}}_{0}^{(m)}, \dot{\mathbf{b}}^{(m)}, \dot{\mathbf{c}}^{(m)}\right)
$$$$
=\frac{1}{m T} \int_{0}^{m T} F_{2}\left(\mathbf{x}^{(m) *}, \mathbf{y}^{(m) *}, t\right) d t
$$$$
=\delta \dot{a}_{20}^{(m)}+\left(\alpha a_{20}^{(m)}+\beta f_{10}^{(m)}\right),
$$$$
F_{2 k}^{(c)}\left(\mathbf{a}_{0}^{(m)}, \mathbf{b}^{(m)}, \mathbf{c}^{(m)}, \dot{\mathbf{a}}_{0}^{(m)}, \dot{\mathbf{b}}^{(m)}, \dot{\mathbf{c}}^{(m)}\right)
$$$$
=\frac{2}{m T} \int_{0}^{m T} F_{2}\left(\mathbf{x}^{(m) *}, \mathbf{y}^{(m) *}, t\right) \cos \left(\frac{k}{m} \Omega t\right) d t
$$$$
=\delta\left(\dot{b}_{2 k / m}+\frac{k \Omega}{m} c_{2 k / m}\right)+\left(\alpha b_{2 k / m}+\beta f_{2 k / m}^{(c)}\right),
$$

$$
\begin{aligned}
F_{2 k}^{(s)} & \left(\mathbf{a}_{0}^{(m)}, \mathbf{b}^{(m)}, \mathbf{c}^{(m)}, \dot{\mathbf{a}}_{0}^{(m)}, \dot{\mathbf{b}}^{(m)}, \dot{\mathbf{c}}^{(m)}\right) \\
= & \frac{2}{m T} \int_{0}^{m T} F_{2}\left(\mathbf{x}^{(m) *}, \mathbf{y}^{(m) *}, t\right) \sin \left(\frac{k}{m} \Omega t\right) d t \\
= & \delta\left(\dot{c}_{2 k / m}-\frac{k \Omega}{m} b_{2 k / m}\right) \\
& +\left(\alpha c_{2 k / m}+\beta f_{2 k / m}^{(s)}\right)-e \Omega^{2} \delta_{k}^{m}
\end{aligned}
$$

The functions for the first oscillator are

$$
\begin{aligned}
f_{10}^{(m)}= & \left(a_{10}^{(m)}\right)^{3}+a_{10}^{(m)}\left(a_{20}^{(m)}\right)^{2} \\
& +\sum_{q=1}^{11} \sum_{l=1}^{N} \sum_{j=1}^{N} \sum_{i=1}^{N} f_{10}^{(m)}(i, j, l, q) \\
f_{1 k / m}^{(c)}= & 3\left(a_{10}^{(m)}\right)^{2} b_{1 k / m}+2 a_{10}^{(m)} a_{20}^{(m)} b_{2 k / m}+\left(a_{20}^{(m)}\right)^{2} b_{1 k / m} \\
& +\sum_{q=1}^{11} \sum_{l=1}^{N} \sum_{j=1}^{N} \sum_{i=1}^{N} f_{1 k / m}^{(c)}(i, j, l, q) \\
f_{1 k / m}^{(s)}= & 3\left(a_{10}^{(m)}\right)^{2} c_{1 k / m}+2 a_{10}^{(m)} a_{20}^{(m)} c_{2 k / m}+\left(a_{20}^{(m)}\right)^{2} c_{1 k / m} \\
& +\sum_{q=1}^{9} \sum_{l=1}^{N} \sum_{j=1}^{N} \sum_{i=1}^{N} f_{1 k / m}^{(s)}(i, j, l, q)
\end{aligned}
$$


with

$$
\begin{aligned}
f_{10}^{(m)}(i, j, l, 1)= & \frac{3 a_{10}^{(m)}}{2 N} b_{1 i / m} b_{1 j / m} \delta_{i-j}^{0} \\
f_{10}^{(m)}(i, j, l, 2)= & \frac{3 a_{10}^{(m)}}{2 N} c_{1 i / m} c_{1 j / m} \delta_{i-j}^{0} \\
f_{10}^{(m)}(i, j, l, 3)= & \frac{1}{4} b_{1 i / m} b_{1 j / m} b_{1 l / m} \\
& \times\left(\delta_{i-j-l}^{0}+\delta_{i-j+l}^{0}+\delta_{i+j-l}^{0}\right) \\
f_{10}^{(m)}(i, j, l, 4)= & \frac{3}{4} b_{1 i / m} c_{1 j / m} c_{1 l / m} \\
& \times\left(\delta_{i+j-l}^{0}+\delta_{i-j+l}^{0}-\delta_{i-j-l}^{0}\right) \\
f_{10}^{(m)}(i, j, l, 5)= & \frac{a_{10}^{(m)}}{2 N} b_{2 i / m} b_{2 j / m} \delta_{i-j}^{0} \\
f_{10}^{(m)}(i, j, l, 6)= & \frac{a_{10}^{(m)}}{2 N} c_{2 i / m} c_{2 j / m} \delta_{i-j}^{0} \\
f_{10}^{(m)}(i, j, l, 7)= & \frac{a_{20}^{(m)}}{N} b_{1 i / m} b_{2 j / m} \delta_{i-j}^{0} \\
f_{10}^{(m)}(i, j, l, 8)= & \frac{a_{20}^{(m)}}{N} c_{1 i / m} c_{2 j / m} \delta_{i-j}^{0} \\
f_{10}^{(m)}(i, j, l, 9)= & \frac{1}{4} b_{1 i / m} b_{2 j / m} b_{2 l / m} \\
& \times\left(\delta_{i-j-l}^{0}+\delta_{i-j+l}^{0}+\delta_{i+j-l}^{0}\right) \\
& \times\left(\delta_{i-j-l}^{0}+\delta_{i-j+l}^{0}-\delta_{i+j-l}^{0}\right) \\
f_{10}^{(m)}(i, j, l, 10)= & \frac{1}{4} b_{1 i / m} c_{2 j / m} c_{2 l / m} \\
& \times\left(\delta_{i+j-l}^{0}+\delta_{i-j+l}^{0}-\delta_{i-j-l}^{0}\right) \\
f_{10}^{(m)}(i, j, l, 11)= & \frac{1}{2} c_{1 i / m} c_{2 j / m} b_{2 l / m} \\
&
\end{aligned}
$$

and

$$
\begin{aligned}
f_{1 k / m}^{(c)}(i, j, l, 1)= & \frac{3 a_{10}^{(m)}}{2 N} b_{1 i / m} b_{1 j / m}\left(\delta_{|i-j|}^{k}+\delta_{i+j}^{k}\right) \\
f_{1 k / m}^{(c)}(i, j, l, 2)= & \frac{3 a_{10}^{(m)}}{2 N} c_{1 i / m} c_{1 j / m}\left(\delta_{|i-j|}^{k}-\delta_{i+j}^{k}\right) \\
f_{1 k / m}^{(c)}(i, j, l, 3)= & \frac{1}{4} b_{1 i / m} b_{1 j / m} b_{1 l / m}\left(\delta_{|i-j-l|}^{k}+\delta_{|i-j+l|}^{k}\right. \\
& \left.+\delta_{|i+j-l|}^{k}+\delta_{i+j+l}^{k}\right) \\
& \left.-\delta_{|i-j-l|}^{k}-\delta_{i+j+l}^{k}\right) \\
f_{1 k / m}^{(c)}(i, j, l, 4)= & \frac{3}{4} b_{1 i / m} c_{1 j / m} c_{1 l / m}\left(\delta_{|i-j+l|}^{k}+\delta_{|i+j-l|}^{k}\right. \\
f_{1 k / m}^{(c)}(i, j, l, 5)= & \frac{a_{10}^{(m)}}{2 N} b_{2 i / m} b_{2 j / m}\left(\delta_{|i-j|}^{k}+\delta_{i+j}^{k}\right) \\
f_{1 k / m}^{(c)}(i, j, l, 6)= & \frac{a_{10}^{(m)}}{2 N} c_{2 i / m} c_{2 j / m}\left(\delta_{|i-j|}^{k}-\delta_{i+j}^{k}\right)
\end{aligned}
$$




$$
\begin{aligned}
& +\delta_{i+j+l}^{k}-\operatorname{sgn}(i+j-l) \delta_{|i+j-l|}^{k} \\
& \left.-\operatorname{sgn}(i-j-l) \delta_{|i-j-l|}^{k}\right] \\
& =\frac{1}{4} b_{2 i / m} b_{2 j / m} c_{1 l / m}\left[\operatorname{sgn}(i-j+l) \delta_{\mid i-j+}^{k}\right. \\
& +\delta_{i+j+l}^{k}-\operatorname{sgn}(i+j-l) \delta_{|i+j-l|}^{k} \\
& \left.-\operatorname{sgn}(i-j-l) \delta_{|i-j-l|}^{k}\right]
\end{aligned}
$$

$f_{1 k / m}^{(s)}(i, j, l, 9)=\frac{1}{4} b_{2 i / m} b_{2 j / m} c_{1 l / m}\left[\operatorname{sgn}(i-j+l) \delta_{|i-j+l|}^{k}\right.$

The functions for the second oscillator are

$$
\begin{aligned}
f_{20}^{(m)}= & \left(a_{20}^{(m)}\right)^{3}+\left(a_{10}^{(m)}\right)^{2} a_{20}^{(m)} \\
& +\sum_{q=1}^{11} \sum_{l=1}^{N} \sum_{j=1}^{N} \sum_{i=1}^{N} f_{20}^{(m)}(i, j, l, q) \\
f_{2 k / m}^{(c)}= & 3\left(a_{20}^{(m)}\right)^{2} b_{2 k / m}+2 a_{10}^{(m)} a_{20}^{(m)} b_{1 k / m}+\left(a_{10}^{(m)}\right)^{2} b_{2 k / m} \\
& +\sum_{q=1}^{11} \sum_{l=1}^{N} \sum_{j=1}^{N} \sum_{i=1}^{N} f_{2 k / m}^{(c)}(i, j, l, q) \\
f_{2 k / m}^{(s)}= & 3\left(a_{20}^{(m)}\right)^{2} c_{2 k / m}+2 a_{10}^{(m)} a_{20}^{(m)} c_{1 k / m}+\left(a_{10}^{(m)}\right)^{2} c_{2 k / m} \\
& +\sum_{q=1}^{9} \sum_{l=1}^{N} \sum_{j=1}^{N} \sum_{i=1}^{N} f_{2 k / m}^{(s)}(i, j, l, q)
\end{aligned}
$$

where

$$
\begin{aligned}
f_{20}^{(m)}(i, j, l, 1)= & \frac{3 a_{20}^{(m)}}{2 N} b_{2 i / m} b_{2 j / m} \delta_{i-j}^{0} \\
f_{20}^{(m)}(i, j, l, 2)= & \frac{3 a_{20}^{(m)}}{2 N} c_{2 i / m} c_{2 j / m} \delta_{i-j}^{0} \\
f_{20}^{(m)}(i, j, l, 3)= & \frac{1}{4} b_{2 i / m} b_{2 j / m} b_{2 l / m} \\
& \times\left(\delta_{i-j-l}^{0}+\delta_{i-j+l}^{0}+\delta_{i+j-l}^{0}\right) \\
f_{20}^{(m)}(i, j, l, 4)= & \frac{3}{4} b_{2 i / m} c_{2 j / m} c_{2 l / m} \\
& \times\left(\delta_{i+j-l}^{0}+\delta_{i-j+l}^{0}-\delta_{i-j-l}^{0}\right) \\
f_{20}^{(m)}(i, j, l, 5)= & \frac{a_{20}^{(m)}}{2 N} b_{2 i / m} b_{2 j / m} \delta_{i-j}^{0} \\
f_{20}^{(m)}(i, j, l, 6)= & \frac{a_{20}^{(m)}}{2 N} c_{1 i / m} c_{1 j / m} \delta_{i-j}^{0} \\
f_{20}^{(m)}(i, j, l, 7)= & \frac{a_{10}^{(m)}}{N} b_{1 i / m} b_{2 j / m} \delta_{i-j}^{0} \\
f_{20}^{(m)}(i, j, l, 8)= & \frac{a_{10}^{(m)}}{N} c_{1 i / m} c_{2 j / m} \delta_{i-j}^{0} \\
f_{20}^{(m)}(i, j, l, 9)= & \frac{1}{4} b_{1 i / m} b_{1 j / m} b_{2 l / m} \\
& \times\left(\delta_{i-j-l}^{0}+\delta_{i-j+l}^{0}+\delta_{i+j-l}^{0}\right)
\end{aligned}
$$

$$
\begin{aligned}
f_{20}^{(m)}(i, j, l, 10)= & \frac{1}{4} b_{2 i / m} c_{1 j / m} c_{1 l / m} \\
& \times\left(\delta_{i+j-l}^{0}+\delta_{i-j+l}^{0}-\delta_{i-j-l}^{0}\right) \\
f_{20}^{(m)}(i, j, l, 11)= & \frac{1}{2} c_{2 i / m} c_{1 j / m} b_{1 l / m} \\
& \left.\times\left(\delta_{i-j-l}^{0}+\delta_{i-j+l}^{0}-\delta_{i+j-l}^{0}\right)\right]
\end{aligned}
$$

and

$$
\begin{aligned}
f_{2 k / m}^{(c)}(i, j, l, 1)= & \frac{3 a_{20}^{(m)}}{2 N} b_{2 i / m} b_{2 j / m}\left(\delta_{|i-j|}^{k}+\delta_{i+j}^{k}\right) \\
f_{2 k / m}^{(c)}(i, j, l, 2)= & \frac{3 a_{20}^{(m)}}{2 N} c_{2 i / m} c_{2 j / m}\left(\delta_{|i-j|}^{k}-\delta_{i+j}^{k}\right) \\
f_{2 k / m}^{(c)}(i, j, l, 3)= & \frac{1}{4} b_{2 i / m} b_{2 j / m} b_{2 l / m}\left(\delta_{|i-j-l|}^{k}\right. \\
& \left.+\delta_{|i-j+l|}^{k}+\delta_{|i+j-l|}^{k}+\delta_{i+j+l}^{k}\right) \\
& \left.+\delta_{|i+j-l|}^{k}-\delta_{|i-j-l|}^{k}-\delta_{i+j+l}^{k}\right) \\
f_{2 k / m}^{(c)}(i, j, l, 4)= & \frac{3}{4} b_{2 i / m} c_{2 j / m} c_{2 l / m}\left(\delta_{|i-j+l|}^{k}\right. \\
f_{2 k / m}^{(c)}(i, j, l, 5)= & \frac{a_{20}^{(m)}}{2 N} b_{1 i / m} b_{1 j / m}\left(\delta_{|i-j|}^{k}+\delta_{i+j}^{k}\right) \\
f_{2 k / m}^{(c)}(i, j, l, 6)= & \frac{a_{20}^{(m)}}{2 N} c_{1 i / m} c_{1 j / m}\left(\delta_{|i-j|}^{k}-\delta_{i+j}^{k}\right) \\
f_{2 k / m}^{(c)}(i, j, l, 7)= & \frac{a_{10}^{(m)}}{N} b_{1 i / m} b_{2 j / m}\left(\delta_{|i-j|}^{k}+\delta_{i+j}^{k}\right) \\
f_{2 k / m}^{(c)}(i, j, l, 8)= & \frac{a_{10}^{(m)}}{N} c_{1 i / m} c_{2 j / m}\left(\delta_{|i-j|}^{k}-\delta_{i+j}^{k}\right) \\
f_{2 k / m}^{(c)}(i, j, l, 9)= & \frac{1}{4} b_{1 i / m} b_{1 j / m} b_{2 l / m}\left(\delta_{|i-j-l|}^{k}+\delta_{|i-j+l|}^{k}\right. \\
& \left.+\delta_{|i+j-l|}^{k}+\delta_{i+j+l}^{k}\right) \\
f_{2 k / m}^{(c)}(i, j, l, 11)= & \frac{1}{2} b_{1 i / m} c_{1 j / m} c_{2 l / m}\left(\delta_{|i-j+l|}^{k}+\delta_{i+j+l}^{k}\right) \\
f_{2 k / m}^{(c)}(i, j, l, 10)= & \frac{1}{4} b_{2 i / m} c_{1 j / m} c_{1 l / m}\left(\delta_{|i-j+l|}^{k}+\delta_{|i+j-l|}^{k}-\delta_{i+j+l}^{k}\right) \\
& \left.\delta_{|i+j|}^{k}\right) \\
& \\
& \\
&
\end{aligned}
$$

and

$$
\begin{aligned}
f_{2 k / m}^{(s)}(i, j, l, 1)= & \frac{3 a_{20}^{(m)}}{N} b_{2 i / m} c_{2 j / m} \\
& \times\left[\delta_{i+j}^{k}-\operatorname{sgn}(i-j) \delta_{|i-j|}^{k}\right] \\
f_{2 k / m}^{(s)}(i, j, l, 2)= & \frac{1}{4} c_{2 i / m} c_{2 j / m} c_{2 l / m}\left[\operatorname{sgn}(i-j+l) \delta_{|i-j+l|}^{k}\right. \\
& +\operatorname{sgn}(i+j-l) \delta_{|i+j-l|}^{k} \\
& \left.-\operatorname{sgn}(i-j-l) \delta_{|i-j-l|}^{k}-\delta_{i+j+l}^{k}\right] \\
f_{2 k / m}^{(s)}(i, j, l, 3)= & \frac{3}{4} b_{2 i / m} b_{2 j / m} c_{2 l / m}\left[\operatorname{sgn}(i-j+l) \delta_{|i-j+l|}^{k}\right.
\end{aligned}
$$




$$
\begin{aligned}
& +\delta_{i+j+l}^{k}-\operatorname{sgn}(i+j-l) \delta_{|i+j-l|}^{k} \\
& \left.-\operatorname{sgn}(i-j-l) \delta_{|i-j-l|}^{k}\right] \\
f_{2 k / m}^{(s)}(i, j, l, 4)= & \frac{a_{10}^{(m)}}{N} b_{1 i / m} c_{2 j / m} \\
& \times\left[\delta_{i+j}^{k}-\operatorname{sgn}(i-j) \delta_{|i-j|}^{k}\right] \\
f_{2 k / m}^{(s)}(i, j, l, 5)= & \frac{a_{10}^{(m)}}{N} c_{1 i / m} b_{2 j / m} \\
& \times\left[\delta_{i+j}^{k}+\operatorname{sgn}(i-j) \delta_{|i-j|}^{k}\right] \\
f_{2 k / m}^{(s)}(i, j, l, 6)= & \frac{a_{20}^{(m)}}{N} b_{1 i / m} c_{1 j / m} \\
& \times\left[\delta_{i+j}^{k}-\operatorname{sgn}(i-j) \delta_{|i-j|}^{k}\right] \\
f_{2 k / m}^{(s)}(i, j, l, 7)= & \frac{1}{4} c_{1 i / m} c_{1 j / m} c_{2 l / m}\left[\operatorname{sgn}(i-j+l) \delta_{|i-j+l|}^{k}\right. \\
& +\operatorname{sgn}(i+j-l) \delta_{|i+j-l|}^{k} \\
& \left.-\operatorname{sgn}(i-j-l) \delta_{|i-j-l|}^{k}-\delta_{i+j+l}^{k}\right] \\
& \frac{1}{2} b_{1 i / m} b_{2 j / m} c_{1 l / m}\left[\operatorname{sgn}(i-j+l) \delta_{|i-j+l|}^{k}\right. \\
& +\delta_{i+j+l}^{k}-\operatorname{sgn}(i+j-l) \delta_{|i+j-l|}^{k} \\
& \left.-\operatorname{sgn}(i-j-l) \delta_{|i-j-l|}^{k}\right] \\
f_{2 k / m}^{(s)}(i, j, l, 9)= & \frac{1}{4} b_{1 i / m} b_{1 j / m} c_{2 l / m}\left[\operatorname{sgn}(i-j+l) \delta_{|i-j+l|}^{k}\right. \\
& \left.+\delta_{i+j+l}^{k}-\operatorname{sgn}(i+j-l) \delta_{|i+j-l|}^{k}(i-j-l) \delta_{|i-j-l|}^{k}\right] \\
f_{2 k / m}^{(s)}(i, j, l, 8) & \\
& \operatorname{sgn}(i+j) \\
&
\end{aligned}
$$

Define

$$
\begin{aligned}
\mathbf{z}^{(m) \triangleq} \triangleq & \left(\mathbf{a}_{0}^{(m)}, \mathbf{b}^{(m)}, \mathbf{c}^{(m)}\right)^{\mathrm{T}} \\
= & \left(a_{10}^{(m)}, b_{11 / m}, \ldots, b_{1 N / m}, c_{11 / m}, \ldots, c_{1 N / m} ;\right. \\
& \left.a_{20}^{(m)}, b_{21 / m}, \ldots, b_{2 N / m}, c_{21 / m}, \ldots, c_{2 N / m}\right)^{\mathrm{T}} \\
\equiv & \left(z_{1}^{(m)}, z_{2}^{(m)}, \ldots, z_{2 N+1}^{(m)} ; z_{2 N+2}^{(m)}, z_{2 N+3}^{(m)}, \ldots, z_{4 N+2}^{(m)}\right)^{\mathrm{T}} \\
\mathbf{z}_{1}^{(m) \triangleq}= & \dot{\mathbf{z}}^{(m)}=\left(\dot{a}_{0}^{(m)}, \dot{\mathbf{b}}^{(m)}, \dot{\mathbf{c}}^{(m)}\right)^{\mathrm{T}} \\
= & \left(\dot{a}_{10}^{(m)}, \dot{b}_{11 / m}, \ldots, \dot{b}_{1 N / m}, \dot{c}_{11 / m}, \ldots, \dot{c}_{1 N / m} ;\right. \\
& \left.\dot{a}_{20}^{(m)}, \dot{b}_{21 / m}, \ldots, \dot{b}_{2 N / m}, \dot{c}_{21 / m}, \ldots, \dot{c}_{2 N / m}\right)^{\mathrm{T}} \\
\equiv & \left(\dot{z}_{1}^{(m)}, \dot{z}_{2}^{(m)}, \ldots, \dot{z}_{2 N+1}^{(m)} ; \dot{z}_{2 N+2}^{(m)}, \dot{z}_{2 N+3}^{(m)}, \ldots, \dot{z}_{4 N+2}^{(m)}\right)^{\mathrm{T}}
\end{aligned}
$$

Equations (8) and (9) are rewritten as

$$
\dot{\mathbf{z}}^{(m)}=\mathbf{z}_{1}^{(m)} \text { and } \dot{\mathbf{z}}_{1}^{(m)}=\mathbf{g}^{(m)}\left(\mathbf{z}^{(m)}, \mathbf{z}_{1}^{(m)}\right)
$$

where

$$
\begin{aligned}
& \mathbf{g}^{(m)}\left(\mathbf{z}^{(m)}, \mathbf{z}_{1}^{(m)}\right) \\
& \quad\left(\begin{array}{l}
-F_{10}^{(m)}\left(\mathbf{z}^{(m)}, \mathbf{z}_{1}^{(m)}\right) \\
-\mathbf{F}_{1 / m}^{(c)}\left(\mathbf{z}^{(m)}, \mathbf{z}_{1}^{(m)}\right)-2 \mathbf{k}_{1} \frac{\Omega}{m} \dot{\mathbf{c}}_{1}^{(m)}+\mathbf{k}_{2}\left(\frac{\Omega}{m}\right)^{2} \mathbf{b}_{1}^{(m)} \\
-\mathbf{F}_{1 / m}^{(s)}\left(\mathbf{z}^{(m)}, \mathbf{z}_{1}^{(m)}\right)+2 \mathbf{k}_{1} \frac{\Omega}{m} \dot{\mathbf{b}}_{1}^{(m)}+\mathbf{k}_{2}\left(\frac{\Omega}{m}\right)^{2} \mathbf{c}_{1}^{(m)} \\
-F_{20}^{(m)}\left(\mathbf{z}^{(m)}, \mathbf{z}_{1}^{(m)}\right) \\
-\mathbf{F}_{2 / m}^{(c)}\left(\mathbf{z}^{(m)}, \mathbf{z}_{1}^{(m)}\right)-2 \mathbf{k}_{1} \frac{\Omega}{m} \dot{\mathbf{c}}_{2}^{(m)}+\mathbf{k}_{2}\left(\frac{\Omega}{m}\right)^{2} \mathbf{b}_{2}^{(m)} \\
-\mathbf{F}_{2 / m}^{(s)}\left(\mathbf{z}^{(m)}, \mathbf{z}_{1}^{(m)}\right)+2 \mathbf{k}_{1} \frac{\Omega}{m} \dot{\mathbf{b}}_{2}^{(m)}+\mathbf{k}_{2}\left(\frac{\Omega}{m}\right)^{2} \mathbf{c}_{2}^{(m)}
\end{array}\right)
\end{aligned}
$$

where

$$
\begin{aligned}
\mathbf{k}_{1} & =\operatorname{diag}(1,2, \ldots, N), \\
\mathbf{k}_{2} & =\operatorname{diag}\left(1,2^{2}, \ldots, N^{2}\right), \\
\mathbf{F}_{1 / m}^{(c)} & =\left(F_{11 / m}^{(c)}, F_{12 / m}^{(c)}, \ldots, F_{1 N / m}^{(s)}\right)^{\mathrm{T}}, \\
\mathbf{F}_{1 / m}^{(s)} & =\left(F_{11 / m}^{(s)}, F_{12 / m}^{(s)}, \ldots, F_{1 N / m}^{(s)}\right)^{\mathrm{T}}, \\
\mathbf{F}_{2 / m}^{(c)} & =\left(F_{21 / m}^{(c)}, F_{22 / m}^{(c)}, \ldots, F_{2 N / m}^{(c)}\right)^{\mathrm{T}}, \\
\mathbf{F}_{2 / m}^{(s)} & =\left(F_{21 / m}^{(s)}, F_{22 / m}^{(s)}, \ldots, F_{2 N / m}^{(s)}\right)^{\mathrm{T}}
\end{aligned}
$$$$
\text { for } N=1,2, \ldots, \infty \text {. }
$$

Setting

$\mathbf{y}^{(m)} \equiv\left(\mathbf{z}^{(m)}, \mathbf{z}_{1}^{(m)}\right)$ and $\mathbf{f}^{(m)}=\left(\mathbf{z}_{1}^{(m)}, \mathbf{g}^{(m)}\right)^{\mathrm{T}}$,

Thus, Eq. (21) becomes

$\dot{\mathbf{y}}^{(m)}=\mathbf{f}^{(m)}\left(\mathbf{y}^{(m)}\right)$.

The steady-state solutions for periodic motion can be obtained by setting $\dot{\mathbf{y}}^{(m)}=\mathbf{0}$, i.e.,

$$
\begin{aligned}
F_{10}^{(m)}\left(\mathbf{z}^{(m)}, \mathbf{z}_{1}^{(m)}\right) & =0 \\
-\mathbf{F}_{1 / m}^{(c)}\left(\mathbf{z}^{(m)}, \mathbf{z}_{1}^{(m)}\right)+\mathbf{k}_{2}\left(\frac{\Omega}{m}\right)^{2} \mathbf{b}_{1}^{(m)} & =0 \\
-\mathbf{F}_{1 / m}^{(s)}\left(\mathbf{z}^{(m)}, \mathbf{z}_{1}^{(m)}\right)+\mathbf{k}_{2}\left(\frac{\Omega}{m}\right)^{2} \mathbf{c}_{1}^{(m)} & =0 \\
F_{20}^{(m)}\left(\mathbf{z}^{(m)}, \mathbf{z}_{1}^{(m)}\right) & =0 \\
-\mathbf{F}_{2 / m}^{(c)}\left(\mathbf{z}^{(m)}, \mathbf{z}_{1}^{(m)}\right)+\mathbf{k}_{2}\left(\frac{\Omega}{m}\right)^{2} \mathbf{b}_{2}^{(m)} & =0 \\
-\mathbf{F}_{2 / m}^{(s)}\left(\mathbf{z}^{(m)}, \mathbf{z}_{1}^{(m)}\right)+\mathbf{k}_{2}\left(\frac{\Omega}{m}\right)^{2} \mathbf{c}_{2}^{(m)} & =0
\end{aligned}
$$

The $(4 N+2)$ nonlinear equations in Eq. (26) are solved by the Newton-Raphson method. In Luo [13], the linearized equation at $\mathbf{y}^{(m) *}=\left(\mathbf{z}^{(m) *}, \mathbf{0}\right)^{\mathrm{T}}$ is

$$
\Delta \dot{\mathbf{y}}^{(m)}=D \mathbf{f}^{(m)}\left(\mathbf{y}^{*(m)}\right) \Delta \mathbf{y}^{(m)}
$$

where

$D \mathbf{f}^{(m)}\left(\mathbf{y}^{*(m)}\right)=\partial \mathbf{f}^{(m)}\left(\mathbf{y}^{(m)}\right) /\left.\partial \mathbf{y}^{(m)}\right|_{\mathbf{y}^{(m) *}}$ 
The corresponding eigenvalues are determined by

$$
\left|D \mathbf{f}^{(m)}\left(\mathbf{y}^{*(m)}\right)-\lambda \mathbf{I}_{4(2 N+1) \times 2(2 N+1)}\right|=0 .
$$

where

$$
D \mathbf{f}\left(\mathbf{y}^{(m) *}\right)=\left[\begin{array}{ll}
\mathbf{0}_{2(2 N+1) \times 2(2 N+1)} & \mathbf{I}_{2(2 N+1) \times 2(2 N+1)} \\
\mathbf{G}_{2(2 N+1) \times(2 N+1)} & \mathbf{H}_{2(2 N+1) \times 2(2 N+1)}
\end{array}\right]
$$

and

$$
\mathbf{G}=\frac{\partial \mathbf{g}^{(m)}}{\partial \mathbf{Z}^{(m)}}=\left(\mathbf{G}^{(10)}, \mathbf{G}^{(1 c)}, \mathbf{G}^{(1 s)}, \mathbf{G}^{(20)}, \mathbf{G}^{(2 c)}, \mathbf{G}^{(2 s)}\right)^{\mathrm{T}}
$$

$$
\begin{aligned}
& \mathbf{G}^{(i 0)}=\left(G_{0}^{(i 0)}, G_{1}^{(i 0)}, \ldots, G_{4 N+1}^{(i 0)}\right), \\
& \mathbf{G}^{(i c)}=\left(\mathbf{G}_{1}^{(i c)}, \mathbf{G}_{2}^{(i c)}, \ldots, \mathbf{G}_{N}^{(i c)}\right)^{\mathrm{T}}, \\
& \mathbf{G}^{(i s)}=\left(\mathbf{G}_{1}^{(1 s)}, \mathbf{G}_{2}^{(i s)}, \ldots, \mathbf{G}_{N}^{(i s)}\right)^{\mathrm{T}}
\end{aligned}
$$

for $i=1,2$; and $N=1,2, \ldots \infty$ with

$$
\begin{aligned}
& \mathbf{G}_{k}^{(i c)}=\left(G_{k 0}^{(i c)}, G_{k 1}^{(i c)}, \ldots, G_{k(4 N+1)}^{(i c)}\right), \\
& \mathbf{G}_{k}^{(i s)}=\left(G_{k 0}^{(i s)}, G_{k 1}^{(i s)}, \ldots, G_{k(4 N+1)}^{(i s)}\right)
\end{aligned}
$$

for $k=1,2, \ldots N$. The corresponding components are

$$
\begin{aligned}
G_{r}^{(10)}= & -(1+\gamma)\left(\alpha \delta_{0}^{r}+\beta g_{r}^{(10)}\right), \\
G_{k r}^{(1 c)}= & \left(\frac{k \Omega}{m}\right)^{2} \delta_{k}^{r}-\delta \frac{k \Omega}{m} \delta_{k+N}^{r}-(1+\gamma) \\
& \times\left(\alpha \delta_{k}^{r}+\beta g_{k r}^{(1 c)}\right), \\
G_{k r}^{(1 s)}= & \left(\frac{k \Omega}{m}\right)^{2} \delta_{k+N}^{r}+\delta \frac{k \Omega}{m} \delta_{k}^{r}-(1+\gamma) \\
& \times\left(\alpha \delta_{k+N}^{r}+\beta g_{k r}^{(1 s)}\right), \\
G_{r}^{(20)}= & -\alpha \delta_{2 N+1}^{r}-\beta g_{r}^{(20)}, \\
G_{k r}^{(2 c)}= & \left(\frac{k \Omega}{m}\right)^{2} \delta_{k+2 N+1}^{r}-\delta \frac{k \Omega}{m} \delta_{k+3 N+1}^{r} \\
& -\alpha \delta_{k+2 N+1}^{r}-\beta g_{k r}^{(2 c)}, \\
G_{k r}^{(2 s)}= & \left(\frac{k \Omega}{m}\right)^{2} \delta_{k+3 N+1}^{r}+\delta \frac{k \Omega}{m} \delta_{k+2 N+1}^{r} \\
& -\alpha \delta_{k+3 N+1}^{r}-\beta g_{k r}^{(2 s)} \\
& \text { for } r=0,1,2, \ldots, 4 N+1
\end{aligned}
$$

where

$$
\begin{aligned}
g_{r}^{(10)} & =3\left(a_{10}^{(m)}\right)^{2} \delta_{0}^{r}+\left(a_{20}^{(m)}\right)^{2} \delta_{0}^{r}+2 a_{10}^{(m)} a_{20}^{(m)} \delta_{2 N+1}^{r} \\
& +\sum_{q=1}^{11} \sum_{l=1}^{N} \sum_{j=1}^{N} \sum_{i=1}^{N} g_{r}^{(10)}(i, j, l, q)
\end{aligned}
$$

with

$$
\begin{aligned}
g_{r}^{(10)}(i, j, l, 1)= & \frac{3}{2 N}\left(b_{1 i / m} b_{1 j / m} \delta_{0}^{r}+2 a_{10}^{(m)} b_{1 i / m} \delta_{j}^{r}\right) \delta_{i-j}^{0} \\
g_{r}^{(10)}(i, j, l, 2)= & \frac{3}{2 N}\left(c_{1 i / m} c_{1 j / m} \delta_{0}^{r}+2 a_{10}^{(m)} c_{1 i / m} \delta_{j+N}^{r}\right) \delta_{i-j}^{0} \\
g_{r}^{(10)}(i, j, l, 3)= & \frac{3}{4} b_{1 i / m} b_{1 j / m} \delta_{l}^{r}\left(\delta_{i-j-l}^{0}+\delta_{i-j+l}^{0}+\delta_{i+j-l}^{0}\right) \\
g_{r}^{(10)}(i, j, l, 4)= & \frac{3}{4}\left(c_{1 j / m} c_{1 l / m} \delta_{i}^{r}+2 b_{1 i / m} c_{1 j / m} \delta_{l+N}^{r}\right)\left(\delta_{i+j-l}^{0}\right. \\
& \left.+\delta_{i-j+l}^{0}-\delta_{i-j-l}^{0}\right) \\
g_{r}^{(10)}(i, j, l, 5)= & \frac{1}{2 N}\left(b_{2 i / m} b_{2 j / m} \delta_{0}^{r}+2 a_{10}^{(m)} b_{2 i / m} \delta_{j+2 N+1}^{r}\right) \delta_{i-j}^{0} \\
g_{r}^{(10)}(i, j, l, 6)= & \frac{1}{2 N}\left(c_{2 i / m} c_{2 j / m} \delta_{0}^{r}+2 a_{10}^{(m)} c_{2 i / m} \delta_{j+3 N+1}^{r}\right) \delta_{i-j}^{0} \\
g_{r}^{(10)}(i, j, l, 7)= & \frac{1}{N}\left(b_{1 i / m} b_{2 j / m} \delta_{2 N+1}^{r}+a_{20}^{(m)} b_{2 j / m} \delta_{i}^{r}\right. \\
& \left.+a_{20}^{(m)} b_{1 i / m} \delta_{j+2 N+1}^{r}\right) \delta_{i-j}^{0} \\
g_{r}^{(10)}(i, j, l, 8)= & \frac{1}{N}\left(c_{1 i / m} c_{2 j / m} \delta_{2 N+1}^{r}+a_{20}^{(m)} c_{2 j / m} \delta_{i+N}^{r}\right. \\
& \left.+a_{20}^{(m)} c_{1 i / m} \delta_{j+3 N+1}^{r}\right) \delta_{i-j}^{0} \\
g_{r}^{(10)}(i, j, l, 9)= & \frac{1}{4}\left(b_{2 j / m} b_{2 l / m} \delta_{i}^{r}+2 b_{1 i / m} b_{2 j / m} \delta_{l+2 N+1}^{r}\right)\left(\delta_{i-j-l}^{0}\right. \\
& \left.+\delta_{i-j+l}^{0}+\delta_{i+j-l}^{0}\right) \\
& \frac{1}{4}\left(c_{2 j / m} c_{2 l / m} \delta_{i}^{r}+2 b_{1 i / m} c_{2 j / m} \delta_{l+3 N+1}^{r}\right)\left(\delta_{i+j-l}^{0}\right. \\
& \left.+\delta_{i-j+l}^{0}-\delta_{i-j-l}^{0}\right) \\
g_{r}^{(10)}(i, j, l, 10) & \frac{1}{2}\left(c_{2 j / m} b_{2 l / m} \delta_{i+N}^{r}+c_{1 i / m} b_{2 l / m} \delta_{j+3 N+1}^{r}+\delta_{i-j+l}^{0}-\delta_{i+j-l}^{0}\right) \\
g_{r}^{(10)}(i, j, l, 11) & \left.c_{1 i / m} c_{2 j / m} \delta_{+2 N+1}^{r}\right) \\
& \times(36)
\end{aligned}
$$

and

$$
\begin{aligned}
g_{k r}^{(1 c)}= & 3\left(a_{10}^{(m)}\right)^{2} \delta_{k}^{r}+6 a_{10}^{(m)} b_{1 k / m} \delta_{0}^{r}+2 a_{20}^{(m)} b_{2 k / m} \delta_{0}^{r} \\
& +2 a_{10}^{(m)} b_{2 k / m} \delta_{2 N+1}^{r}+2 a_{10}^{(m)} a_{20}^{(m)} \delta_{k+2 N+1}^{r} \\
& +2 a_{20}^{(m)} b_{1 k / m} \delta_{2 N+1}^{r}+\left(a_{20}^{(m)}\right)^{2} \delta_{k}^{r} \\
& +\sum_{q=1}^{11} \sum_{l=1}^{N} \sum_{j=1}^{N} \sum_{i=1}^{N} g_{k r}^{(1 c)}(i, j, l, q)
\end{aligned}
$$

with

$$
\begin{aligned}
g_{k r}^{(1 c)}(i, j, l, 1)= & \frac{3}{2 N}\left(b_{1 i / m} b_{1 j / m} \delta_{0}^{r}+2 a_{01}^{(m)} b_{1 i / m} \delta_{j}^{r}\right)\left(\delta_{|i-j|}^{k}+\delta_{i+j}^{k}\right) \\
g_{k r}^{(1 c)}(i, j, l, 2)= & \frac{3}{2 N}\left(c_{1 i / m} c_{1 j / m} \delta_{0}^{r}+2 a_{10}^{(m)} c_{1 i / m} \delta_{j+N}^{r}\right)\left(\delta_{|i-j|}^{k}-\delta_{i+j}^{k}\right) \\
g_{k r}^{(1 c)}(i, j, l, 3)= & \frac{3}{4} b_{1 i / m} b_{1 j / m} \delta_{l}^{r}\left(\delta_{|i-j-l|}^{k}+\delta_{|i-j+l|}^{k}\right. \\
& \left.\quad+\delta_{|i+j-l|}^{k}+\delta_{|i+j+l|}^{k}\right) \\
g_{k r}^{(1 c)}(i, j, l, 4)= & \frac{3}{4}\left(c_{1 j / m} c_{1 l / m} \delta_{i}^{r}+2 b_{1 i / m} c_{1 j / m} \delta_{l+N}^{r}\right) \\
& \times\left(\delta_{|i-j+l|}^{k}+\delta_{|i+j-l|}^{k}-\delta_{|i-j-l|}^{k}-\delta_{i+j+l}^{k}\right)
\end{aligned}
$$


$g_{k r}^{(1 c)}(i, j, l, 5)=\frac{1}{2 N}\left(b_{2 i / m} b_{2 j / m} \delta_{0}^{r}+2 a_{10}^{(m)} b_{2 i / m} \delta_{j+2 N+1}^{r}\right)$

$$
\left(\delta_{|i-j|}^{k}+\delta_{i+j}^{k}\right)
$$

$g_{k r}^{(1 c)}(i, j, l, 6)=\frac{1}{2 N}\left(c_{2 i / m} c_{2 j / m} \delta_{0}^{r}+a_{10}^{(m)} c_{2 i / m} \delta_{j+3 N+1}^{r}\right)$

$$
\left(\delta_{|i-j|}^{k}-\delta_{i+j}^{k}\right)
$$

$g_{k r}^{(1 c)}(i, j, l, 7)=\frac{1}{N}\left(b_{1 i / m} b_{2 j / m} \delta_{2 N+1}^{r}+a_{20}^{(m)} b_{2 j / m} \delta_{i}^{r}\right.$

$$
\left.+a_{20}^{(m)} b_{1 i / m} \delta_{j+2 N+1}^{r}\right)\left(\delta_{|i-j|}^{k}+\delta_{i+j}^{k}\right)
$$

$g_{k r}^{(1 c)}(i, j, l, 8)=\frac{1}{N}\left(c_{1 i / m} c_{2 j / m} \delta_{2 N+1}^{r}+a_{20}^{(m)} c_{2 j / m} \delta_{i+N}^{r}\right.$

$$
\left.+a_{20}^{(m)} c_{1 i / m} \delta_{j+3 N+1}^{r}\right)\left(\delta_{|i-j|}^{k}-\delta_{i+j}^{k}\right)
$$

$g_{k r}^{(1 c)}(i, j, l, 9)=\frac{1}{4}\left(b_{2 j / m} b_{2 l / m} \delta_{i}^{r}+2 b_{1 i / m} b_{2 j / m} \delta_{l+2 N+1}^{r}\right)$

$$
\times\left(\delta_{|i-j-l|}^{k}+\delta_{|i-j+l|}^{k}+\delta_{|i+j-l|}^{k}+\delta_{|i+j+l|}^{k}\right)
$$

$g_{k r}^{(1 c)}(i, j, l, 10)=\frac{1}{4}\left(c_{2 j / m} c_{2 l / m} \delta_{i}^{r}+2 b_{1 i / m} c_{2 j / m} \delta_{l+3 N+1}^{r}\right)$

$$
\times\left(\delta_{|i-j+l|}^{k}+\delta_{|i+j-l|}^{k}-\delta_{|i-j-l|}^{k}-\delta_{i+j+l}^{k}\right)
$$

$g_{k r}^{(1 c)}(i, j, l, 11)=\frac{1}{2}\left(c_{2 j / m} c_{1 l / m} \delta_{i+2 N+1}^{r}+b_{2 i / m} c_{1 l / m} \delta_{j+3 N+1}^{r}\right.$

$$
\begin{aligned}
& \left.+b_{2 i / m} c_{2 j / m} \delta_{l+N}^{r}\right)\left(\delta_{|i-j+l|}^{k}+\delta_{|i+j-l|}^{k}\right. \\
& \left.-\delta_{|i-j-l|}^{k}-\delta_{i+j+l}^{k}\right)
\end{aligned}
$$

and

$$
\begin{aligned}
g_{k r}^{(1 s)}= & 3\left(a_{10}^{(m)}\right)^{2} \delta_{k+N}^{r}+6 a_{10}^{(m)} c_{1 k / m} \delta_{0}^{r}+2 a_{20}^{(m)} c_{2 k / m} \delta_{0}^{r} \\
& +2 a_{10}^{(m)} c_{2 k / m} \delta_{2 N+1}^{r}+2 a_{10}^{(m)} a_{20}^{(m)} \delta_{k+3 N+1}^{r} \\
& +2 a_{20}^{(m)} c_{1 k / m} \delta_{2 N+1}^{r}+\left(a_{20}^{(m)}\right)^{2} \delta_{k+N}^{r} \\
& +\sum_{q=1}^{9} \sum_{l=1}^{N} \sum_{j=1}^{N} \sum_{i=1}^{N} g_{k r}^{(1 s)}(i, j, l, q)
\end{aligned}
$$

with

$$
\begin{aligned}
g_{k r}^{(1 s)}(i, j, l, 1)= & \frac{3}{N}\left(a_{10}^{(m)} c_{1 j / m} \delta_{i}^{r}+a_{10}^{(m)} b_{1 i / m} \delta_{j+N}^{r}\right. \\
& \left.+b_{1 i / m} c_{1 j / m} \delta_{0}^{r}\right) \\
& \times\left[\delta_{i+j}^{k}-\operatorname{sgn}(i-j) \delta_{|i-j|}^{k}\right] \\
g_{k r}^{(1 s)}(i, j, l, 2)= & \frac{3}{4} c_{1 i / m} c_{1 j / m} \delta_{l+N}^{r}\left[\operatorname{sgn}(i-j+l) \delta_{|i-j+l|}^{k}\right. \\
& +\operatorname{sgn}(i+j-l) \delta_{|i+j-l|}^{k} \\
& \left.-\operatorname{sgn}(i-j-l) \delta_{|i-j-l|}^{k}-\delta_{i+j+l}^{k}\right]
\end{aligned}
$$

$g_{k r}^{(1 s)}(i, j, l, 3)=\frac{3}{4}\left(b_{1 i / m} b_{1 j / m} \delta_{l+N}^{r}+2 b_{1 i / m} c_{1 l / m} \delta_{j}^{r}\right)$

$$
\begin{aligned}
& {\left[\operatorname{sgn}(i-j+l) \delta_{|i-j+l|}^{k}\right.} \\
& +\delta_{i+j+l}^{k}-\operatorname{sgn}(i+j-l) \delta_{|i+j-l|}^{k} \\
& \left.-\operatorname{sgn}(i-j-l) \delta_{|i-j-l|}^{k}\right]
\end{aligned}
$$

$$
\begin{aligned}
& g_{k r}^{(1 s)}(i, j, l, 4)=\frac{1}{N}\left(a_{20}^{(m)} c_{2 j / m} \delta_{i}^{r}+a_{20}^{(m)} b_{1 i / m} \delta_{j+3 N+1}^{r}\right. \\
& \left.+b_{1 i / m} c_{2 j / m} \delta_{2 N+1}^{r}\right) \times\left[\delta_{i+j}^{k}-\operatorname{sgn}(i-j) \delta_{|i-j|}^{k}\right] \\
& g_{k r}^{(1 s)}(i, j, l, 5)=\frac{1}{N}\left(a_{20}^{(m)} b_{2 j / m} \delta_{i+N}^{r}+a_{20}^{(m)} c_{1 i / m} \delta_{j+2 N+1}^{r}\right. \\
& \left.\quad+c_{1 i / m} b_{2 j / m} \delta_{2 N+1}^{r}\right) \times\left[\delta_{i+j}^{k}+\operatorname{sgn}(i-j) \delta_{|i-j|}^{k}\right]
\end{aligned}
$$$$
g_{k r}^{(1 s)}(i, j, l, 6)=\frac{1}{N}\left(a_{10}^{(m)} c_{2 j / m} \delta_{i+2 N+1}^{r}+a_{10}^{(m)} b_{2 i / m} \delta_{j+3 N+1}^{r}\right.
$$$$
\left.+b_{2 i / m} c_{y j / m} \delta_{0}^{r}\right) \times\left[\delta_{i+j}^{k}-\operatorname{sgn}(i-j) \delta_{|i-j|}^{k}\right]
$$

$g_{k r}^{(1 s)}(i, j, l, 7)=\frac{1}{4}\left(c_{2 j / m} c_{2 l / m} \delta_{i+N}^{r}+2 c_{1 i / m} c_{2 j / m} \delta_{l+3 N+1}^{r}\right)$

$$
\times\left[\operatorname{sgn}(i-j+l) \delta_{|i-j+l|}^{k}+\operatorname{sgn}(i+j-l) \delta_{|i+j-l|}^{k}\right.
$$$$
\left.-\operatorname{sgn}(i-j-l) \delta_{|i-j-l|}^{k}-\delta_{i+j+l}^{k}\right]
$$

$g_{k r}^{(1 s)}(i, j, l, 8)=\frac{1}{2}\left(b_{2 j / m} c_{2 l / m} \delta_{i}^{r}+b_{1 i / m} c_{2 l / m} \delta_{j+2 N+1}^{r}\right.$

$\left.+b_{1 i / m} b_{2 j / m} \delta_{l+3 N+1}^{r}\right) \times\left[\operatorname{sgn}(i-j+l) \delta_{|i-j+l|}^{k}+\delta_{i+j+l}^{k}\right.$

$\left.-\operatorname{sgn}(i+j-l) \delta_{|i+j-l|}^{k}-\operatorname{sgn}(i-j-l) \delta_{|i-j-l|}^{k}\right]$

$g_{k r}^{(1 s)}(i, j, l, 9)=\frac{1}{4}\left(2 b_{2 j / m} c_{1 l / m} \delta_{i+2 N+1}^{r}+b_{2 i / m} b_{2 j / m} \delta_{l+N}^{r}\right)$

$$
\times\left[\operatorname{sgn}(i-j+l) \delta_{|i-j+l|}^{k}+\delta_{i+j+l}^{k}-\operatorname{sgn}(i+j-l) \delta_{|i+j-l|}^{k}\right.
$$$$
\left.-\operatorname{sgn}(i-j-l) \delta_{|i-j-l|}^{k}\right]
$$

and

$$
\begin{aligned}
g_{r}^{(20)}= & 3\left(a_{20}^{(m)}\right)^{2} \delta_{2 N+1}^{r}+\left(a_{10}^{(m)}\right)^{2} \delta_{2 N+1}^{r}+2 a_{10}^{(m)} a_{20}^{(m)} \delta_{0}^{r} \\
& +\sum_{q=1}^{11} \sum_{l=1}^{N} \sum_{j=1}^{N} \sum_{i=1}^{N} g_{r}^{(20)}(i, j, l, q)
\end{aligned}
$$

with

$$
\begin{aligned}
g_{r}^{(20)}(i, j, l, 1)= & \frac{3}{2 N}\left(b_{2 i / m} b_{2 j / m} \delta_{2 N+1}^{r}+2 a_{20}^{(m)} b_{2 i / m} \delta_{j+2 N+1}^{r}\right) \delta_{i-j}^{0}, \\
g_{r}^{(20)}(i, j, l, 2)= & \frac{3}{2 N}\left(c_{2 i / m} c_{2 j / m} \delta_{2 N+1}^{r}+2 a_{20}^{(m)} c_{2 i / m} \delta_{j+3 N+1}^{r}\right) \delta_{i-j}^{0}, \\
g_{r}^{(20)}(i, j, l, 3)= & \frac{3}{4} b_{2 i / m} b_{2 j / m} \delta_{l+2 N+1}^{r}\left(\delta_{i-j-l}^{0}+\delta_{i-j+l}^{0}+\delta_{i+j-l}^{0}\right), \\
g_{r}^{(20)}(i, j, l, 4)= & \frac{3}{4}\left(c_{2 j / m} c_{2 l / m} \delta_{i+2 N+1}^{r}+2 b_{2 i / m} c_{2 j / m} \delta_{l+3 N+1}^{r}\right) \\
& \times\left(\delta_{i+j-l}^{0}+\delta_{i-j+l}^{0}-\delta_{i-j-l}^{0}\right), \\
g_{r}^{(20)}(i, j, l, 5)= & \frac{1}{2 N}\left(b_{1 i / m} b_{1 j / m} \delta_{2 N+1}^{r}+2 a_{20}^{(m)} b_{1 i / m} \delta_{j}^{r}\right) \delta_{i-j}^{0}, \\
g_{r}^{(20)}(i, j, l, 6)= & \frac{1}{2 N}\left(c_{1 i / m} c_{1 j / m} \delta_{2 N+1}^{r}+2 a_{20}^{(m)} c_{1 i / m} \delta_{j+N}^{r}\right) \delta_{i-j}^{0}, \\
g_{r}^{(20)}(i, j, l, 7)= & \frac{1}{N}\left(b_{1 i / m} b_{2 j / m} \delta_{r}^{0}+a_{10}^{(m)} b_{1 i / m} \delta_{j+2 N+1}^{r}\right. \\
& \left.+a_{01}^{(m)} b_{y j / m} \delta_{i}^{r}\right) \delta_{i-j}^{0}, \\
g_{r}^{(20)}(i, j, l, 8)= & \frac{1}{N}\left(c_{1 i / m} c_{2 j / m} \delta_{r}^{0}+a_{10}^{(m)} c_{1 i / m} \delta_{j+3 N+1}^{r}\right. \\
& \left.+a_{10}^{(m)} c_{2 j / m} \delta_{i+N}^{r}\right) \delta_{i-j}^{0},
\end{aligned}
$$




$$
\begin{aligned}
& g_{r}^{(20)}(i, j, l, 9)= \frac{1}{4}\left(2 b_{1 j / m} b_{2 l / m} \delta_{i}^{r}+b_{1 i / m} b_{1 j / m} \delta_{l+2 N+1}^{r}\right) \\
& \times\left(\delta_{i-j-l}^{0}+\delta_{i-j+l}^{0}+\delta_{i+j-l}^{0}\right), \\
& g_{r}^{(20)}(i, j, l, 10)= \frac{1}{4}\left(c_{1 j / m} c_{1 l / m} \delta_{i+2 N+1}^{r}+2 b_{2 i / m} c_{1 j / m} \delta_{l+N}^{r}\right) \\
& \times\left(\delta_{i+j-l}^{0}+\delta_{i-j+l}^{0}-\delta_{i-j-l}^{0}\right), \\
& g_{r}^{(20)}(i, j, l, 11)= \frac{1}{2}\left(c_{1 j / m} b_{1 l / m} \delta_{i+3 N+1}^{r}+c_{2 i / m} b_{1 l / m} \delta_{j+N}^{r}\right. \\
&\left.+c_{2 i / m} c_{1 j / m} \delta_{l}^{r}\right)\left(\delta_{i-j-l}^{0}+\delta_{i-j+l}^{0}-\delta_{i+j-l}^{0}\right) . \\
& \text { and } \quad \\
& g_{k r}^{(2 c)}=3\left(a_{20}^{(m)}\right)^{2} \delta_{k+2 N+1}^{r}+6 a_{20}^{(m)} b_{2 k / m} \delta_{2 N+1}^{r}+2 a_{20}^{(m)} b_{1 k / m} \delta_{0}^{r} \\
&+2 a_{01}^{(m)} b_{1 k / m} \delta_{2 N+1}^{r}+2 a_{10}^{(m)} a_{20}^{(m)} \delta_{k}^{r}+2 a_{10}^{(m)} b_{2 k / m} \delta_{0}^{r} \\
&+\left(a_{10}^{(m)}\right)^{2} \delta_{k+2 N+1}^{r}+\sum_{q=1}^{11} \sum_{l=1}^{N} \sum_{j=1}^{N} \sum_{i=1}^{N} g_{k r}^{(2 c)}(i, j, l, q)
\end{aligned}
$$

with

$$
\begin{aligned}
g_{k r}^{(2 c)}(i, j, l, 1)= & \frac{3}{2 N}\left(b_{2 i / m} b_{2 j / m} \delta_{2 N+1}^{r}\right. \\
& \left.+2 a_{20}^{(m)} b_{2 i / m} \delta_{j+2 N+1}^{r}\right)\left(\delta_{|i-j|}^{k}+\delta_{i+j}^{k}\right) \\
g_{k r}^{(2 c)}(i, j, l, 2)= & \frac{3}{2 N}\left(c_{2 i / m} c_{2 j / m} \delta_{2 N+1}^{r}\right. \\
& \left.+2 a_{20}^{(m)} c_{2 i / m} \delta_{j+3 N+1}^{r}\right)\left(\delta_{|i-j|}^{k}-\delta_{i+j}^{k}\right) \\
g_{k r}^{(2 c)}(i, j, l, 3)= & \frac{3}{4} b_{2 i / m} b_{2 j / m} \delta_{l+2 N+1}^{r}\left(\delta_{|i-j-l|}^{k}+\delta_{|i-j+l|}^{k}\right. \\
& \left.+\delta_{|i+j-l|}^{k}+\delta_{|i+j+l|}^{k}\right)
\end{aligned}
$$

$g_{k r}^{(2 c)}(i, j, l, 4)=\frac{3}{4}\left(c_{2 j / m} c_{2 l / m} \delta_{i+2 N+1}^{r}+2 b_{2 i / m} c_{2 j / m} \delta_{l+3 N+1}^{r}\right)$$$
\times\left(\delta_{|i-j+l|}^{k}+\delta_{|i+j-l|}^{k}-\delta_{|i-j-l|}^{k}-\delta_{i+j+l}^{k}\right)
$$$$
g_{k r}^{(2 c)}(i, j, l, 5)=\frac{1}{2 N}\left(b_{1 i / m} b_{1 j / m} \delta_{2 N+1}^{r}\right.
$$$$
\left.+2 a_{20}^{(m)} b_{1 i / m} \delta_{j}^{r}\right)\left(\delta_{|i-j|}^{k}+\delta_{i+j}^{k}\right)
$$

$g_{k r}^{(2 c)}(i, j, l, 6)=\frac{1}{2 N}\left(c_{1 i / m} c_{1 j / m} \delta_{2 N+1}^{r}\right.$

$$
\left.+2 a_{20}^{(m)} c_{1 i / m} \delta_{j+N}^{r}\right)\left(\delta_{|i-j|}^{k}-\delta_{i+j}^{k}\right)
$$

$g_{k r}^{(2 c)}(i, j, l, 7)=\frac{1}{N}\left(b_{1 i / m} b_{2 j / m} \delta_{0}^{r}\right.$

$$
\begin{aligned}
& \left.+a_{10}^{(m)} b_{2 j / m} \delta_{i}^{r}+a_{10}^{(m)} b_{1 i / m} \delta_{j+2 N+1}^{r}\right) \\
& \times\left(\delta_{|i-j|}^{k}+\delta_{i+j}^{k}\right)
\end{aligned}
$$

$g_{k r}^{(2 c)}(i, j, l, 8)=\frac{1}{N}\left(c_{1 i / m} c_{2 j / m} \delta_{0}^{r}+a_{10}^{(m)} c_{2 j / m} \delta_{i+N}^{r}\right.$

$$
\left.+a_{01}^{(m)} c_{1 i / m} \delta_{j+3 N+1}^{r}\right) \times\left(\delta_{|i-j|}^{k}-\delta_{i+j}^{k}\right)
$$

$g_{k r}^{(2 c)}(i, j, l, 9)=\frac{1}{4}\left(2 b_{1 j / m} b_{2 l / m} \delta_{i}^{r}+b_{1 i / m} b_{1 j / m} \delta_{l+2 N+1}^{r}\right)$

$$
\begin{aligned}
& \times\left(\delta_{|i-j-l|}^{k}+\delta_{|i-j+l|}^{k}+\delta_{|i+j-l|}^{k}+\delta_{|i+j+l|}^{k}\right) \\
g_{k r}^{(2 c)}(i, j, l, 10)= & \frac{1}{4}\left(c_{1 j / m} c_{1 l / m} \delta_{i+2 N+1}^{r}+2 b_{2 i / m} c_{1 j / m} \delta_{l+N}^{r}\right) \\
& \times\left(\delta_{|i-j+l|}^{k}+\delta_{|i+j-l|}^{k}-\delta_{|i-j-l|}^{k}-\delta_{i+j+l}^{k}\right) \\
g_{k r}^{(2 c)}(i, j, l, 11)= & \frac{1}{2}\left(c_{1 j / m} c_{2 l / m} \delta_{i}^{r}\right. \\
& \left.+b_{1 i / m} c_{2 l / m} \delta_{j+N}^{r}+b_{1 i / m} c_{1 j / m} \delta_{l+3 N+1}^{r}\right) \\
& \times\left(\delta_{|i-j+l|}^{k}+\delta_{|i+j-l|}^{k}-\delta_{|i-j-l|}^{k}-\delta_{i+j+l}^{k}\right)
\end{aligned}
$$

and

$$
\begin{aligned}
g_{k r}^{(2 s)}= & 3\left(a_{20}^{(m)}\right)^{2} \delta_{k+3 N+1}^{r}+6 a_{20}^{(m)} c_{y k / m} \delta_{2 N+1}^{r}+2 a_{20}^{(m)} c_{1 k / m} \delta_{r}^{0} \\
& +2 a_{10}^{(m)} c_{1 k / m} \delta_{2 N+1}^{r}+2 a_{10}^{(m)} a_{20}^{(m)} \delta_{k+N}^{r}+2 a_{10}^{(m)} c_{2 k / m} \delta_{0}^{r} \\
& +\left(a_{10}^{(m)}\right)^{2} \delta_{k+3 N+1}^{r}+\sum_{q=1}^{9} \sum_{l=1}^{N} \sum_{j=1}^{N} \sum_{i=1}^{N} g_{k r}^{(2 s)}(i, j, l, q)
\end{aligned}
$$

with

$$
\begin{aligned}
& g_{k r}^{(2 s)}(i, j, l, 1)=\frac{3}{N}\left(a_{20}^{(m)} c_{2 j / m} \delta_{i+2 N+1}^{r}+a_{20}^{(m)} b_{2 i / m} \delta_{j+3 N+1}^{r}\right. \\
& \left.+b_{2 i / m} c_{2 j / m} \delta_{2 N+1}^{r}\right) \\
& \times\left[\delta_{i+j}^{k}-\operatorname{sgn}(i-j) \delta_{|i-j|}^{k}\right] \\
& g_{k r}^{(2 s)}(i, j, l, 2)=\frac{3}{4} c_{2 i / m} c_{2 j / m} \delta_{l+3 N+1}^{r}\left[\operatorname{sgn}(i-j+l) \delta_{|i-j+l|}^{k}\right. \\
& +\operatorname{sgn}(i+j-l) \delta_{|i+j-l|}^{k} \\
& \left.-\operatorname{sgn}(i-j-l) \delta_{|i-j-l|}^{k}-\delta_{i+j+l}^{k}\right] \\
& g_{k r}^{(2 s)}(i, j, l, 3)=\frac{3}{4}\left(b_{2 i / m} b_{2 j / m} \delta_{l+3 N+1}^{r}\right. \\
& \left.+2 b_{2 i / m} c_{2 l / m} \delta_{j+2 N+1}^{r}\right) \\
& \times\left[\operatorname{sgn}(i-j+l) \delta_{|i-j+l|}^{k}+\delta_{i+j+l}^{k}\right. \\
& -\operatorname{sgn}(i+j-l) \delta_{|i+j-l|}^{k} \\
& \left.-\operatorname{sgn}(i-j-l) \delta_{|i-j-l|}^{k}\right] \\
& g_{k r}^{(2 s)}(i, j, l, 4)=\frac{1}{N}\left(a_{10}^{(m)} c_{2 j / m} \delta_{i}^{r}\right. \\
& \left.+a_{10}^{(m)} b_{1 i / m} \delta_{j+3 N+1}^{r}+b_{1 i / m} c_{2 j / m} \delta_{0}^{r}\right) \\
& \times\left[\delta_{i+j}^{k}-\operatorname{sgn}(i-j) \delta_{|i-j|}^{k}\right] \\
& g_{k r}^{(2 s)}(i, j, l, 5)=\frac{1}{N}\left(a_{10}^{(m)} b_{2 j / m} \delta_{i+N}^{r}+a_{10}^{(m)} c_{1 i / m} \delta_{j+2 N+1}^{r}\right. \\
& \left.+c_{1 i / m} b_{2 j / m} \delta_{0}^{r}\right) \\
& \times\left[\delta_{i+j}^{k}+\operatorname{sgn}(i-j) \delta_{|i-j|}^{k}\right] \\
& g_{k r}^{(2 s)}(i, j, l, 6)=\frac{1}{N}\left(a_{20}^{(m)} c_{1 j / m} \delta_{i}^{r}+a_{20}^{(m)} b_{1 i / m} \delta_{j+N}^{r}\right. \\
& \left.+b_{1 i / m} c_{1 j / m} \delta_{2 N+1}^{r}\right) \\
& \times\left[\delta_{i+j}^{k}-\operatorname{sgn}(i-j) \delta_{|i-j|}^{k}\right]
\end{aligned}
$$




$$
\begin{aligned}
g_{k r}^{(2 s)}(i, j, l, 7)= & \frac{1}{4}\left(2 c_{1 j / m} c_{2 l / m} \delta_{i+N}^{r}\right. \\
& \left.+c_{1 i / m} c_{1 j / m} \delta_{l+3 N+1}^{r}\right) \\
& \times\left[\operatorname{sgn}(i-j+l) \delta_{|i-j+l|}^{k}\right. \\
& +\operatorname{sgn}(i+j-l) \delta_{|i+j-l|}^{k} \\
& \left.-\operatorname{sgn}(i-j-l) \delta_{|i-j-l|}^{k}-\delta_{i+j+l}^{k}\right] \\
g_{k r}^{(2 s)}(i, j, l, 8)= & \frac{1}{2}\left(b_{2 j / m} c_{1 l / m} \delta_{i}^{r}+b_{1 i / m} c_{1 l / m} \delta_{j+2 N+1}^{r}\right. \\
& \left.+b_{1 i / m} b_{2 j / m} \delta_{l+N}^{r}\right) \\
& \times\left[\operatorname{sgn}(i-j+l) \delta_{|i-j+l|}^{k}+\delta_{i+j+l}^{k}\right. \\
& -\operatorname{sgn}(i+j-l) \delta_{|i+j-l|}^{k} \\
& \left.-\operatorname{sgn}(i-j-l) \delta_{|i-j-l|}^{k}\right] \\
& \frac{1}{4}\left(2 b_{1 j / m} c_{2 l / m} \delta_{i}^{r}+b_{1 i / m} b_{1 j / m} \delta_{l+3 N+1}^{r}\right) \\
& \times\left[\operatorname{sgn}(i-j+l) \delta_{|i-j+l|}^{k}\right. \\
& \left.+\delta_{i+j+l}^{k}-\operatorname{sgn}(i-j-l) \delta_{|i-j-l|}^{k}\right] \\
g_{k r}^{(2 s)}(i, j, l, 9) & \\
&
\end{aligned}
$$

and

$$
\mathbf{H}=\frac{\partial \mathbf{g}^{(m)}}{\partial \mathbf{z}_{1}^{(m)}}=\left(\mathbf{H}^{(10)}, \mathbf{H}^{(1 c)}, \mathbf{H}^{(1 s)}, \mathbf{H}^{(20)}, \mathbf{H}^{(2 c)}, \mathbf{H}^{(2 s)}\right)^{\mathrm{T}}
$$

where

$$
\begin{aligned}
\mathbf{H}^{(i 0)} & =\left(H_{0}^{(i 0)}, H_{1}^{(i 0)}, \ldots, H_{4 N+1}^{(i 0)}\right), \\
\mathbf{H}^{(i c)} & =\left(\mathbf{H}_{1}^{(i c)}, \mathbf{H}_{2}^{(i c)}, \ldots, \mathbf{H}_{N}^{(i c)}\right)^{\mathrm{T}}, \\
\mathbf{H}^{(i s)} & =\left(\mathbf{H}_{1}^{(i s)}, \mathbf{H}_{2}^{(i s)}, \ldots, \mathbf{H}_{N}^{(i s)}\right)^{\mathrm{T}}
\end{aligned}
$$

for $i=1,2$ and $N=1,2, \ldots \infty$, with

$$
\begin{aligned}
\mathbf{H}_{k}^{(i c)} & =\left(H_{k 0}^{(i c)}, H_{k 1}^{(i c)}, \ldots, H_{k(4 N+1)}^{(i c)}\right), \\
\mathbf{H}_{k}^{(i s)} & =\left(H_{k 0}^{(i s)}, H_{k 1}^{(i s)}, \ldots, H_{k(4 N+1)}^{(i s)}\right)
\end{aligned}
$$

for $k=1,2, \ldots N$. The corresponding components are

$$
\begin{aligned}
& H_{r}^{(10)}=-\delta \delta_{0}^{r}, \\
& H_{k r}^{(1 c)}=-2 \frac{k \Omega}{m} \delta_{k+N}^{r}-\delta \delta_{k}^{r}, \\
& H_{k r}^{(1 s)}=2 \frac{k \Omega}{m} \delta_{k}^{r}-\delta \delta_{k+N}^{r}, \\
& H_{r}^{(20)}=-\delta \delta_{2 N+1}^{r},
\end{aligned}
$$

$$
\begin{aligned}
& H_{k r}^{(2 c)}=-2 \frac{k \Omega}{m} \delta_{k+3 N+1}^{r}-\delta \delta_{k+2 N+1}^{r}, \\
& H_{k r}^{(2 s)}=2 \frac{k \Omega}{m} \delta_{k+2 N+1}^{r}-\delta \delta_{k+3 N+1}^{r} \\
& \text { for } r=0,1, \ldots, 4 N+1 .
\end{aligned}
$$

From Luo [13], the eigenvalues of $D \mathbf{f}^{(m)}\left(\mathbf{y}^{*(m)}\right)$ are classified as

$\left(n_{1}, n_{2}, n_{3} \mid n_{4}, n_{5}, n_{6}\right)$

The corresponding boundary between the stable and unstable solutions is given by the saddle-node bifurcation and Hopf bifurcation.

\section{Frequency-amplitude characteristics}

The curves of harmonic amplitude varying with excitation frequency $\Omega$ are illustrated. The corresponding solution in Eq. (4) can be re-written as

$$
\begin{aligned}
& x^{*}(t)=a_{10}^{(m)}+\sum_{k=1}^{N} A_{(1) k / m} \cos \left(\frac{k}{m} \Omega t-\varphi_{(1) k / m}\right), \\
& y^{*}(t)=a_{20}^{(m)}+\sum_{k=1}^{N} A_{(2) k / m} \cos \left(\frac{k}{m} \Omega t-\varphi_{(2) k / m}\right),
\end{aligned}
$$

where the harmonic amplitude and phase are defined by

$$
A_{(i) k / m} \equiv \sqrt{b_{i k / m}^{2}+c_{i k / m}^{2}}, \varphi_{(i) k / m}=\arctan \frac{c_{i k / m}}{b_{i k / m}}
$$

The system parameters are

$$
\delta=0.05, \quad \alpha=0.68, \quad \beta=10, \quad \gamma=1.0, \quad e=1.5
$$

The acronyms "USN" and "SN" are used to represent the saddle-unstable node and saddle-node bifurcations, respectively. The acronyms "UHB" and "HB" are used to represent the unstable Hopf bifurcation (subcritical) and stable Hopf bifurcation (supercritical), respectively. Solid and dashed curves represent stable and unstable period-m motions, respectively.

\subsection{Period-1 motions}

From the above parameters, the frequency-amplitude curves of period- 1 motions in $x$-direction and $y$-direction of the rotor are presented in Figs. 1 and 2 that are based on 13 harmonic terms.

In Fig. 1, the period-1 motion of the nonlinear rotor in the $x$-direction is presented. In Fig. 1(i), the constant $a_{10}$ versus rotation speed $\Omega$ is presented. For the symmetric period-1 motion, $a_{10}=0$ is observed. For the asymmetric period1 motion, the rotation speed lies in the approximate range of $\Omega \in(0.883,2.017)$. From the symmetric to asymmetric 


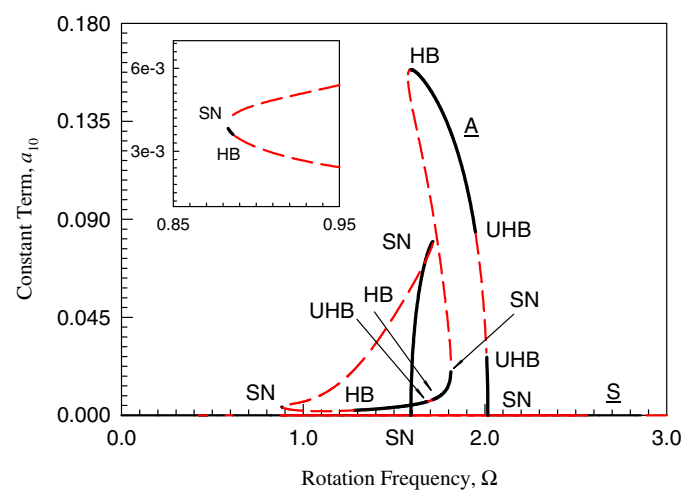

(i)

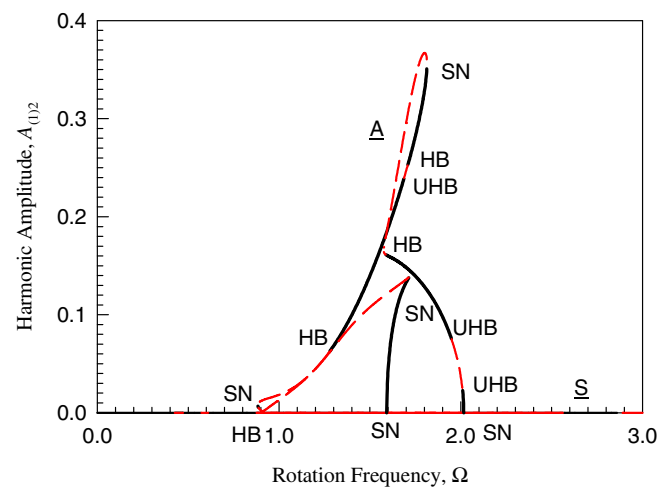

(iii)

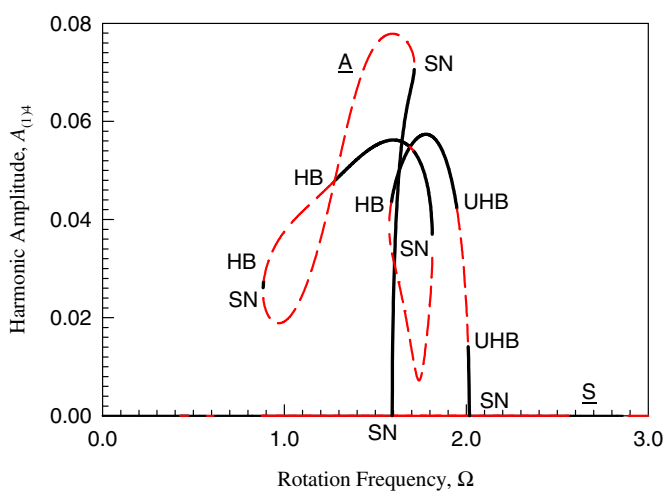

(v)

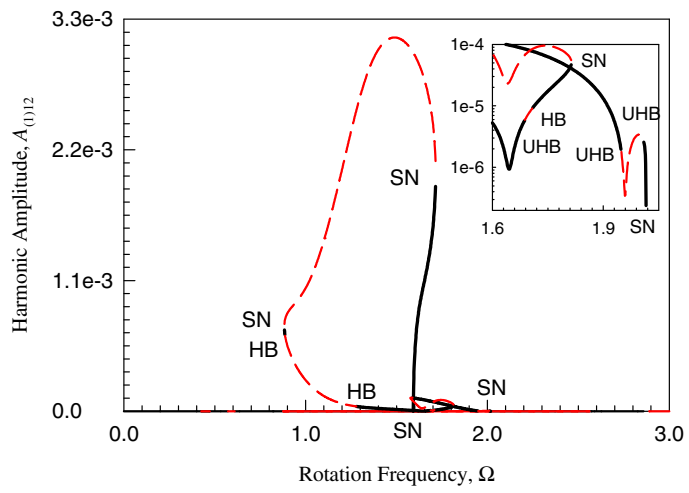

(vii)

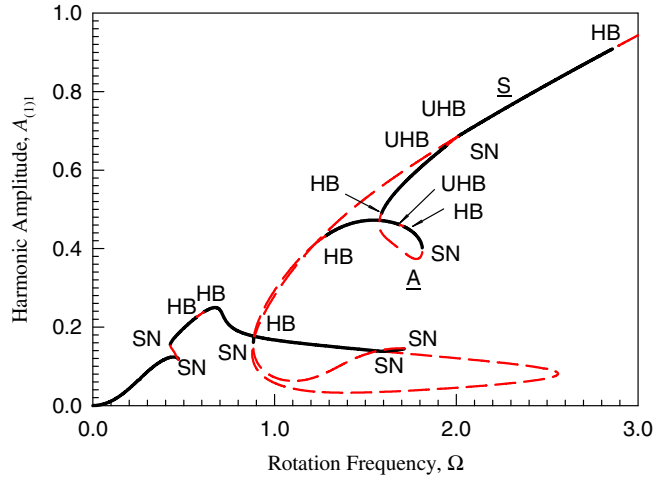

(ii)

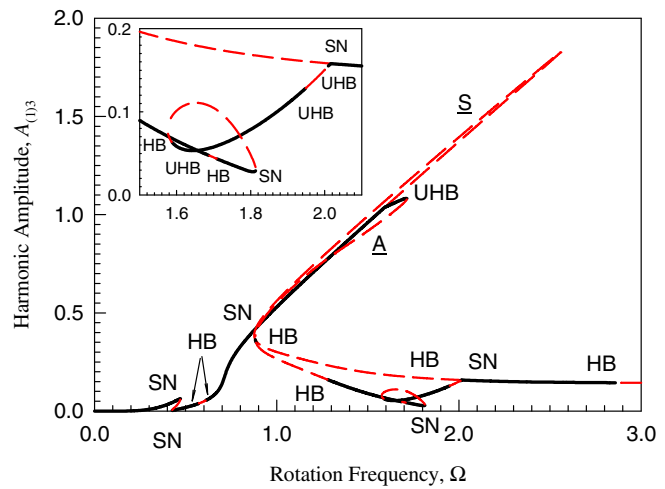

(iv)

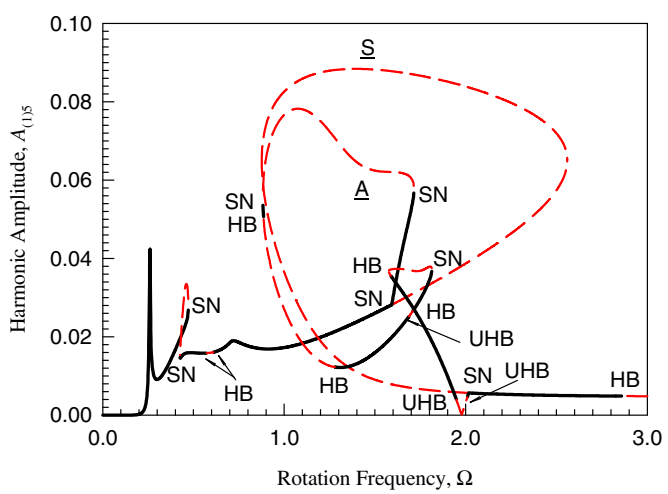

(vi)

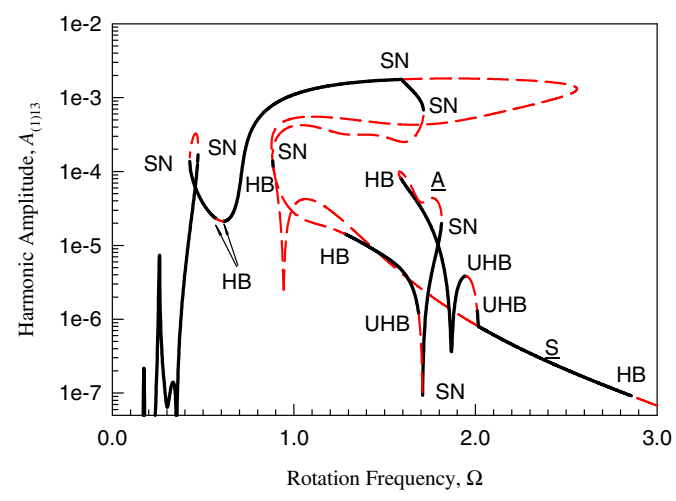

(viii)

Fig. 1 Period-1 motion of the $x$-direction in the nonlinear rotor: frequency-amplitude curves of harmonic terms based on 13 harmonic terms (HB13): (i) $a_{10}$, (ii)-(viii) $A_{(1) k}(k=1,2, \ldots 5,12,13),(\delta=0.02, \alpha=0.68, \beta=10, \gamma=1.0, e=1.5)$ 

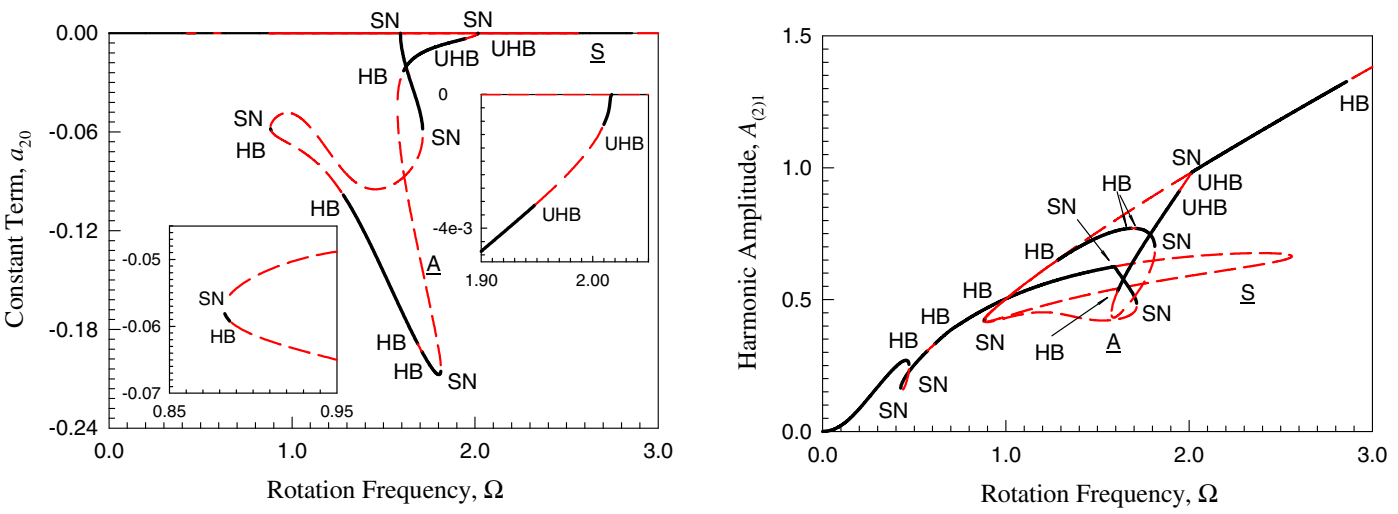

(i)

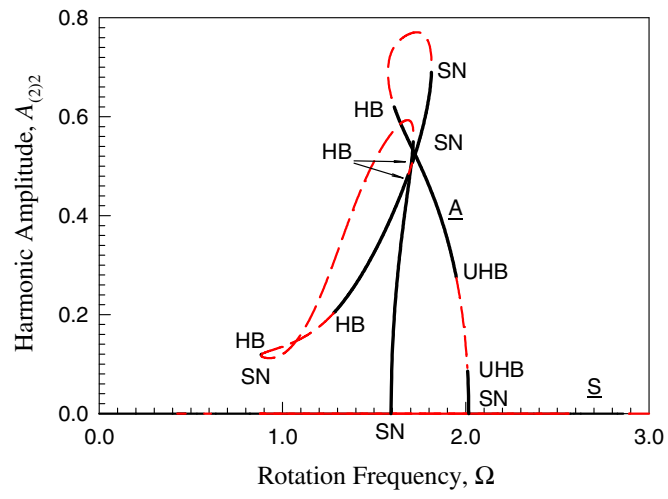

(iii)

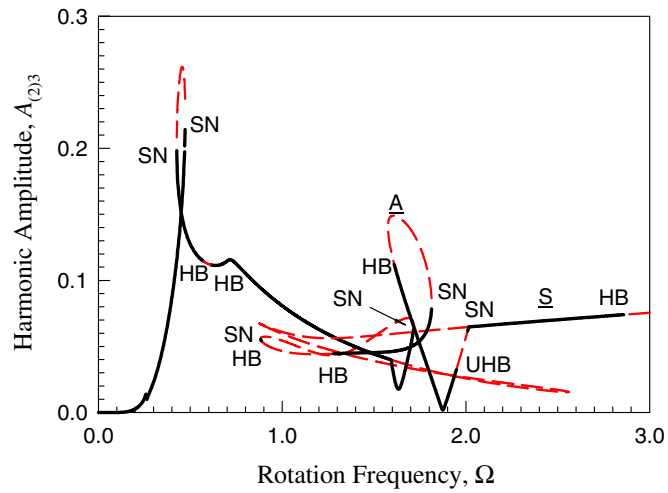

(iv)
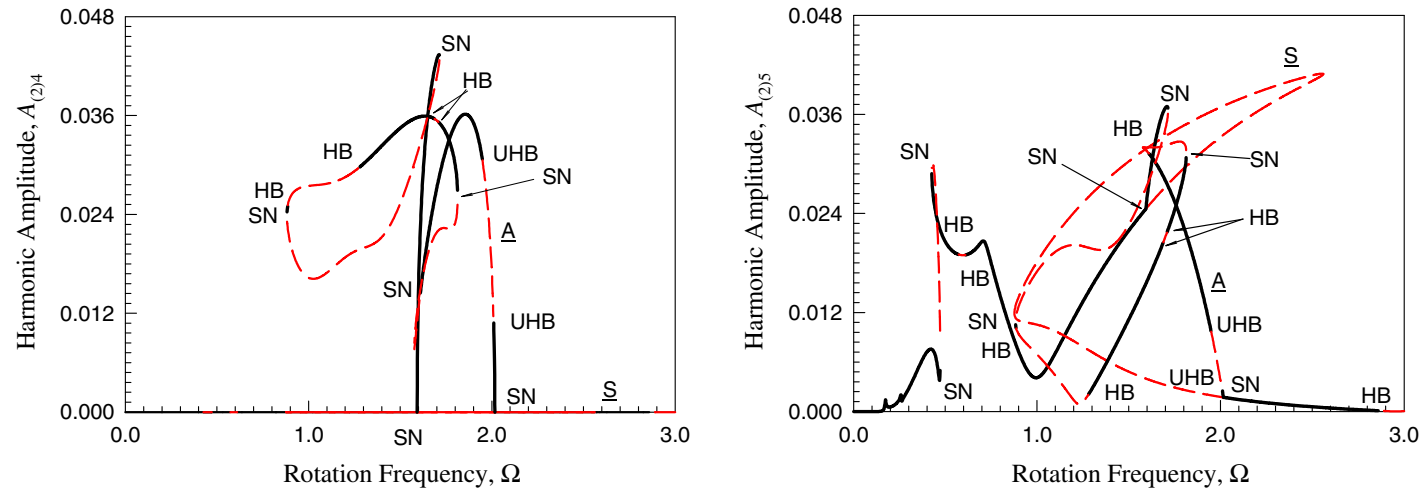

(v)

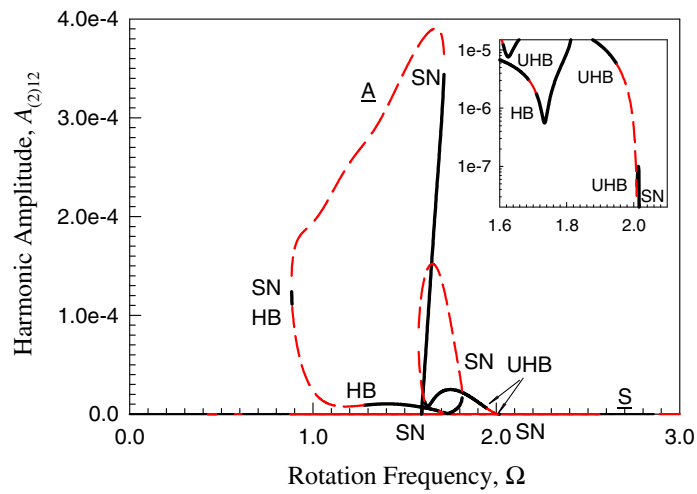

(vii)

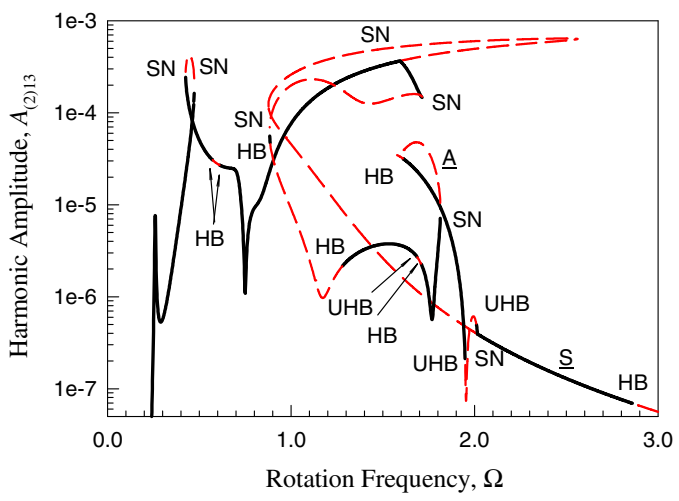

(viii)

Fig. 2 Period-1 motion of the $y$-direction in the nonlinear rotor: frequency-amplitude curves of harmonic terms based on 13 harmonic terms (HB13): (i) $a_{20}$, (ii)-(viii) $A_{(2) k}(k=1,2, \ldots 5,12,13),(\delta=0.02, \alpha=0.68, \beta=10, \gamma=1.0, e=1.5)$ 
period-1 motion, the two saddle-node bifurcations occur at $\Omega \approx 1.592,2.017$. The asymmetric period- 1 motion possesses the unstable Hopf bifurcation (UHB), Hopf bifurcation (HB), and saddle-node bifurcation ( $\mathrm{SN}$ ), respectively. The unstable Hopf bifurcations (UHB) of the asymmetric period-1 motion are located at $\Omega \approx 1.687,1.94865,2.01$. The stable Hopf bifurcations (HB) of the asymmetric period1 motion occur at $\Omega \approx 0.895,1.28,1.58722,1.709$. The other saddle-node bifurcations of the asymmetric period-1 motion are at $\Omega \approx 0.883,1.714,1.813$. The range of the constant is $a_{10}<0.18$. The stable asymmetric period- 1 motion has 6 segments and the unstable asymmetric period- 1 motion possesses 5 segments. One of six segments is very short with $\Omega \approx(0.883,0.895)$ and $a_{10} \sim 4 \times 10^{-3}$, which is zoomed. Only positive constant of $a_{10}>0$ is presented from which the center of the period- 1 motion is on the positive $x$-axis. The constant of $a_{10}<0$ for asymmetric period- 1 motion will not be presented. Such a constant has the same magnitude and the center of the period-1 motion is on the negative $x$-axis. In Fig. 1(ii), the harmonic amplitude $A_{1}$ varying with rotation speed $\Omega$ is presented. In addition to the stable and unstable Hopf bifurcations and saddle-node bifurcations for the asymmetric period-1 motion, the Hopf bifurcation and saddle-node bifurcations for symmetric period-1 motion can be determined. The saddle-node bifurcations for symmetric period- 1 motion are at $\Omega \approx 0.426,0.472$. The Hopf bifurcations of the symmetric period-1 motion occur at $\Omega \approx 0.572,0.611,2.859$. From such Hopf bifurcation, the quasi-periodic motions are observed. Based on the traditional analysis, one may obtain the approximate frequency-amplitude curves for symmetric motion. The harmonic amplitudes for the symmetric and asymmetric period-1 motions are presented with $A_{1(1)}<1.0$. In Fig. 1(iii), the harmonic amplitude $A_{(1) 2}$ versus rotation speed $\Omega$ are presented. Only asymmetric period- 1 motion exists as for constant $a_{10}$ with $A_{(1) 2}<0.4$. For symmetric period-1 motion, $A_{(1) 2}=0$ is observed. In Fig. 1(iv), the harmonic amplitude $A_{(1) 3}$ varying with rotation frequency is presented. For asymmetric motion, its local view is zoomed for the stability detail. The harmonic amplitude $A_{(1) 3}<2.0$ possesses largest values compared to the primary harmonic amplitude $A_{(1) 1}<1.0$. The harmonic amplitude $A_{1(4)}$ versus rotation speed $\Omega$ is presented in Fig. 1(v). The quantity level of $A_{1(4)}$ drops to $A_{1(4)} \sim 8 \times 10^{-2}$ from $A_{(1) 3} \sim 2.0$. In Fig. 1(vi), the harmonic amplitude $A_{(1) 5}$ versus rotation speed $\Omega$ is presented, and the quantity level of $A_{(1) 5}$ with $A_{1(4)}$ is quite close to $A_{(1) 5} \sim 10^{-1}$. To avoid abundant illustrations, the harmonic amplitudes $A_{(1) 12}$ and $A_{(1) 13}$ are presented in Fig. 1(vii), (viii), respectively. The quantity levels of $A_{(1) 12}$ and $A_{(1) 13}$ are close to $10^{-3}$. As usual, the more harmonic terms should be included. The local view of asymmetric period-1 motion is zoomed in Fig. 1(vii), and the logarithm scale is used to present the harmonic amplitude of $A_{(1) 13}$ in Fig. 1(viii).
For this nonlinear rotor system, the period-1 motion of the nonlinear rotor in the $y$-direction is presented in Fig. 2. In Fig. 2(i), the constant term $a_{20}$ versus rotation speed $\Omega$ is presented. Only the asymmetric period- 1 motion exists as in the $x$-direction, but the values of $a_{20}$ lie in the range of $a_{20} \in(-0.24,0)$. Two local areas are zoomed to view the details. The positive $a_{20}$ can be obtained with mirror symmetry as for negative $a_{10}$. The harmonic amplitude $A_{(2) 1}$ varying with rotation speed is presented in Fig. 2(ii), and the primary harmonic amplitude in the $y$-direction is $A_{(2) 1} \in(0,1.5)$ different from $A_{(1) 1}$ in the $x$-direction. The symmetric and asymmetric period-1 motions are presented. In Fig. 2(iii), the harmonic amplitude $A_{(2) 2}$ versus rotation speed $\Omega$ is depicted only for the asymmetric period- 2 motion with $A_{(2) 2} \sim 1$ because the symmetric period- 1 motion has $A_{(2) 2}=0$. The harmonic amplitude $A_{(2) 3}$ versus rotation speed is presented in Fig. 2(iv). The quantity level of $A_{(2) 3}$ is about $A_{(2) 3} \sim 0.3$ much less than $A_{(1) 3} \sim 2$. The harmonic amplitudes $A_{(2) 4}$ and $A_{(2) 5}$ varying with rotation speed are shown in Fig. 2(v), (vi), respectively. The quantity levels of the two harmonic amplitudes are $A_{(2) 4} \sim 0.05$ and $A_{(2) 5} \sim 0.05$. To avoid abundant illustrations, the harmonic amplitudes $A_{(2) 12}$ and $A_{(2) 13}$ are presented in Fig. 2(vii), (viii), respectively. Their quantity levels are $A_{(12) 12} \sim 4 \times 10^{-4}$ and $A_{(2) 13} \sim 10^{-3}$.

\subsection{Bifurcation trees}

From the Hopf bifurcations of symmetric period-1 motion, the quasi-periodic motion or other periodic motions may exist. However, for asymmetric period-1 motion, its Hopf bifurcation may cause period-2 motions. For the stable Hopf bifurcation, the stable period-2 motion will be obtained. For the unstable Hopf bifurcation, the unstable period-2 motion will be achieved. The analytical solutions of period- 2 motions are based on the 26 harmonic terms (HB26) in the Fourier series solution.

In Fig. 3, the first branch of bifurcation tree of the period-1 motion to period- 2 motion of the nonlinear rotor in the $x$ direction is presented. The constant term $a_{10}^{(m)}(m=1,2)$ versus rotation speed is presented in Fig. 3(i). Two local areas are zoomed to show the bifurcation characteristics. The saddle-node bifurcations of period- 2 motions occur at $\Omega \approx 1.548,2.01022$ and the Hopf bifurcation of period-2 motion occurs at $\Omega \approx 1.5870$. The unstable Hopf bifurcation of unstable asymmetric period-1 motion occurs at $\Omega \approx 1.5874$. The unstable period- 2 motions appear from the unstable Hopf bifurcations. The positive constant $a_{10}^{(m)}$ lies in the range of $a_{10}^{(m)} \in(0,0.18)$. The bifurcation relation of period-1 to period- 2 motion is clearly illustrated. For period-2 motion, harmonic amplitude $A_{(1) 1 / 2}$ is presented in Fig. 3(ii). The appearances of period- 2 motions take place at 


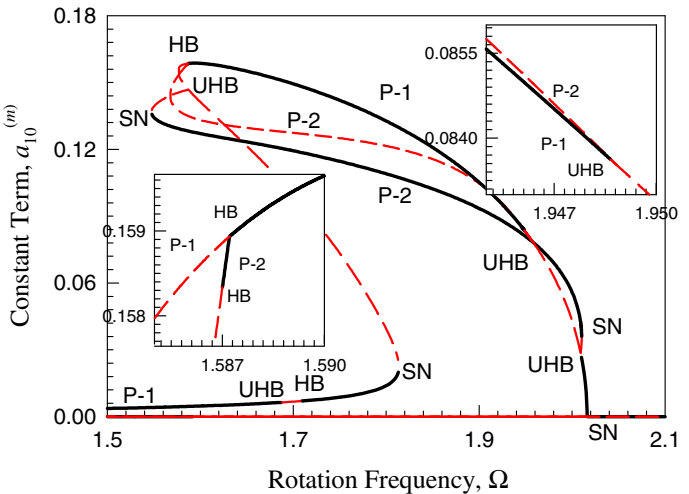

(i)

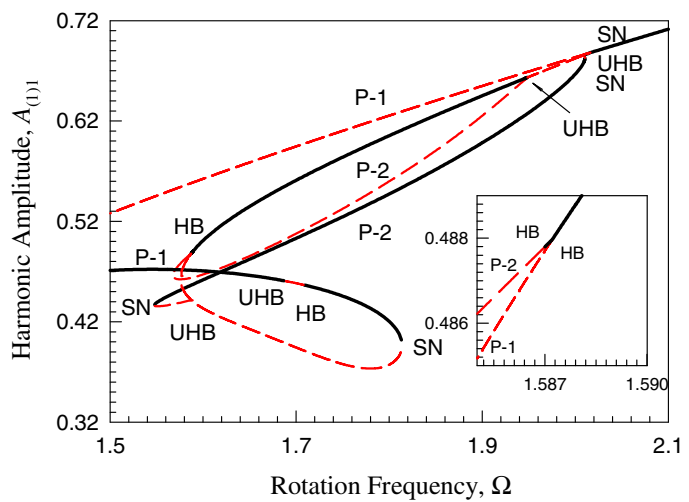

(iii)

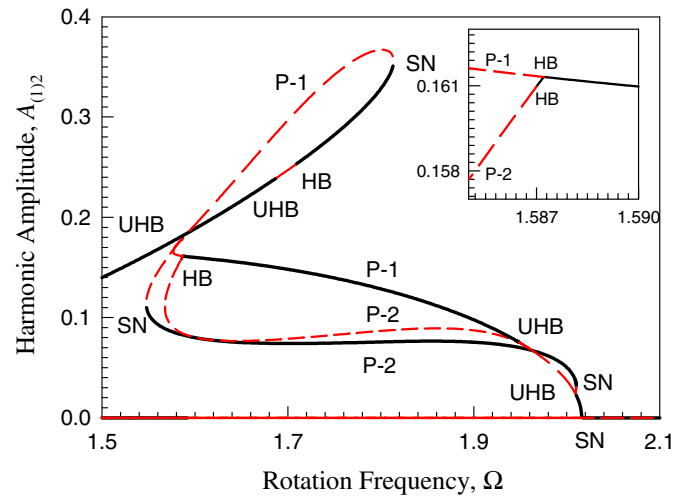

(v)

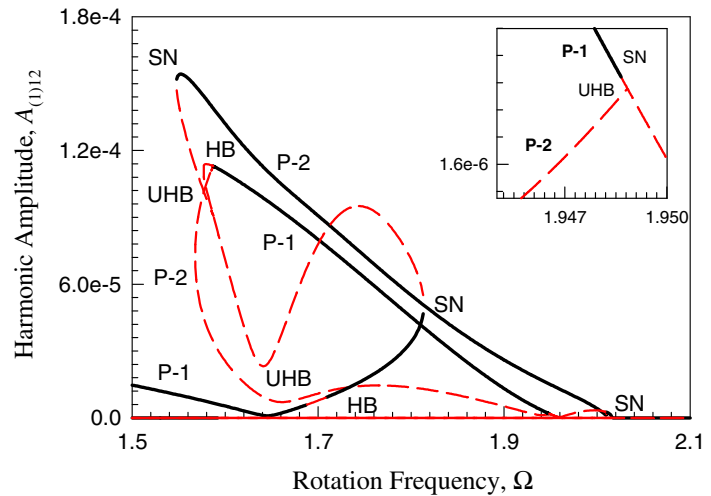

(vii)

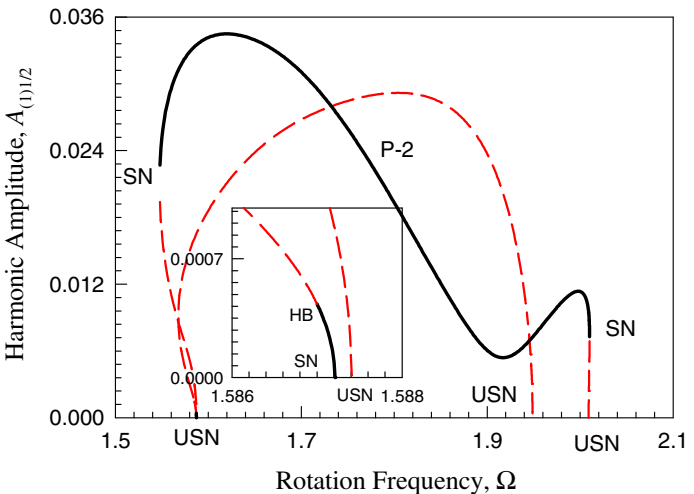

(ii)

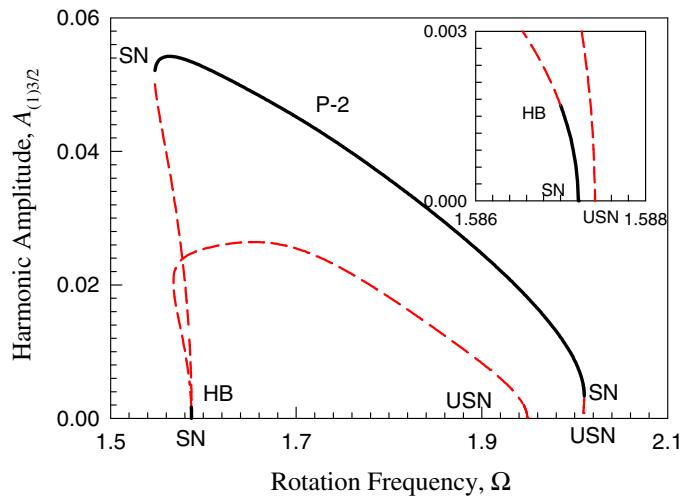

(iv)

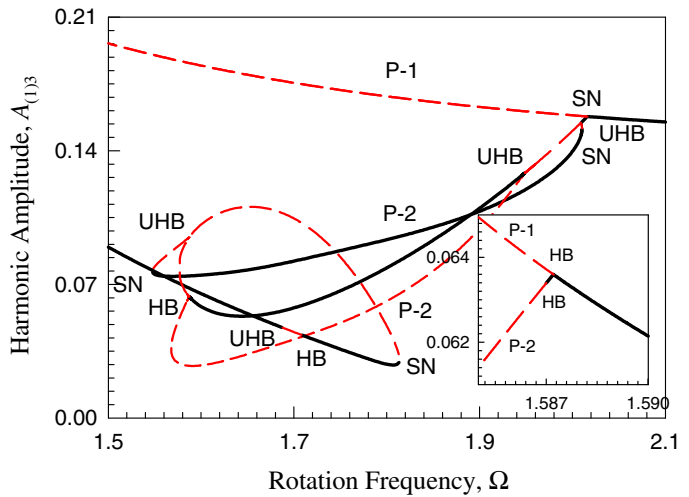

(vi)

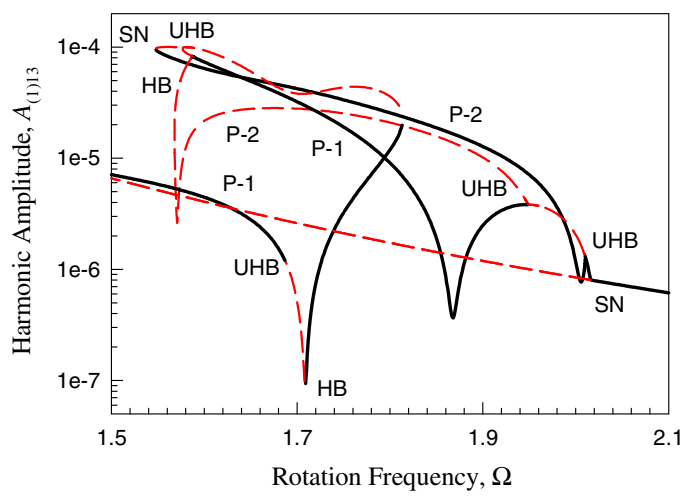

(viii)

Fig. 3 Bifurcation tree of period-1 motion to chaos of the $x$-direction in the nonlinear rotor: frequency-amplitude curves of harmonic terms based on 26 harmonic terms: (i) $a_{10}^{(m)}$, (ii)-(viii) $A_{(1) k / m}(k=1,2, \ldots, 4,6,24,26, m=2),(\delta=0.02, \alpha=0.68, \beta=10, \gamma=1.0, e=1.5)$ 
the Hopf bifurcation of period-1 motions, which is also the saddle-node bifurcation of period-2 motion for appearance at $\Omega \approx 1.58722$. The unstable Hopf bifurcations (UHB) of period-1 motion give the unstable saddle-node bifurcations (USN) of period-2 motions for the onset of unstable period2 motion. The unstable saddle-node bifurcations (USN) of period- 2 motions are at $\Omega \approx 1.5874,1.94865,2.01$. The quantity level of $A_{(1) 1 / 2} \sim 4 \times 10^{-2}$ is observed and the period-2 motion for this branch is in the range of $\Omega \in$ $(1.5,2.1)$. The harmonic amplitude $A_{(1) 1}$ versus rotation speed is presented in Fig. 3(iii) for the bifurcation tree of period-1 motion to period-2 motion via the Hopf bifurcations. The quantity level of $A_{(1) 1}$ for period- 2 motion is in the range of $A_{(1) 1} \sim 0.7$ for $\Omega \in(1.5,2.1)$. Compared to $A_{(1) 1 / 2}$, the harmonic amplitude $A_{(1) 3 / 2}$ versus rotation speed is presented in Fig. 3(iv) with $A_{(1) 3 / 2} \sim 6 \times 10^{-2}$ in $\Omega \in(1.5,2.1)$. Compared to the period- 1 motion, the harmonic amplitudes $A_{(1) 2}$ and $A_{(1) 3}$ versus rotation speed are illustrated in Fig. 3(v) and (vi), respectively. Their quantity levels are $A_{(1) 2} \sim 0.4$ and $A_{(1) 3} \sim 0.2$. In Fig. 3(v), no symmetric period-1 motion exists, and both asymmetric period-1 motion and period-2 motion exist to show the bifurcation tree much clearly. Such an illustration is like constant term in Fig. 3(i). For harmonic amplitude $A_{(1) 3}$, symmetric and asymmetric period- 1 motion and period- 2 motion coexist. So the illustrations will be very crowded. So only the area related to period-2 motion is presented in Fig. 3(vi). To reduce abundant illustrations, the harmonic amplitudes $A_{(1) 12}$ and $A_{(1) 13}$ versus rotation speed are illustrated in Fig. 3 (vii), (viii), respectively. The quantity levels for the two harmonic amplitudes for period- 2 motions are $A_{(1) 12} \sim 2 \times 10^{-4}$ and $A_{(1) 13} \sim 1 \times 10^{-4}$.

As in Fig. 3, the first branch of bifurcation tree of the period-1 motion to period-2 motion of the nonlinear rotor in the $y$-direction is presented in Fig. 4. In Fig. 4(i), the constant term $a_{20}^{(m)}(m=1,2)$ versus rotation speed is presented. A local area is zoomed to show the bifurcation characteristics. The bifurcation points are the same as discussed in Fig. 3. The negative constant $a_{20}^{(m)}$ lies in the range of $a_{20}^{(m)} \in(-0.24,0)$. The bifurcation relation of period-1 to period-2 motion is clearly illustrated. For period-2 motion, the harmonic amplitude $A_{(2) 1 / 2}$ in the $y$-direction is presented in Fig. 4(ii), which is different from the harmonic amplitude in the $x$-direction. The locations of bifurcations are the same as in Fig. 3. The quantity level of $A_{(2) 1 / 2} \sim 5 \times 10^{-2}$ is observed in the range of $\Omega \in(1.5,2.1)$. The harmonic amplitude $A_{(2) 1}$ varying with rotation speed is presented in Fig. 4(iii) for the bifurcation tree of period-1 motion to period-2 motion. The quantity level of $A_{(2) 1} \sim 1$ for period2 motion is observed for $\Omega \in(1.5,2.1)$. The harmonic amplitude $A_{(2) 3 / 2}$ versus rotation speed is presented in Fig. 4(iv) with $A_{(2) 3 / 2} \sim 5 \times 10^{-2}$. The harmonic amplitudes
$A_{(2) 2}$ and $A_{(2) 3}$ versus rotation speed are illustrated in Fig. 4(v), (vi), respectively. The quantity levels of $A_{(2) 2} \sim 1$ and $A_{(2) 3} \sim 0.2$ are observed. To reduce abundant illustrations, the harmonic amplitudes $A_{(1) 12}$ and $A_{(1) 13}$ versus rotation speed are illustrated in Fig. 4(vii), (viii), respectively. The quantity levels for the two harmonic amplitudes for period- 2 motions are $A_{(2) 12} \sim 1.6 \times 10^{-4}$ and $A_{(2) 13} \sim 1 \times 10^{-4}$.

In Fig. 5, the second branch of bifurcation tree of the period-1 motion to period- 2 motion of the nonlinear rotor in the $x$-direction is presented in $\Omega \in(1.30,1.80)$, which is much simpler than the first branch of bifurcation tree in $\Omega \in(1.5,2.1)$. Only the asymmetric period-1 motion relative to period-2 motion is presented herein. The constant term $a_{10}^{(m)}(m=1,2)$ versus rotation speed is presented in Fig. 5(i). The Hopf bifurcation of period-1 motion occurs at $\Omega \approx 1.70872$ and the Hopf bifurcation of period-2 motion occurs at $\Omega \approx 1.5875$. The unstable Hopf bifurcation of unstable asymmetric period- 1 motion occurs at $\Omega \approx 1.689$. The unstable period- 2 motions appears from the unstable Hopf bifurcation of period-1 motion. The positive constant $a_{10}^{(m)}$ lies in the range of $a_{10}^{(m)} \in(0,0.015)$. The bifurcation relation of period- 1 to period- 2 motion is clearly illustrated. For period-2 motion, harmonic amplitude $A_{(1) 1 / 2}$ is presented in Fig. 5(ii). The onset of period-2 motions takes place at the Hopf bifurcation of period-1 motions, which is the saddle-node bifurcation of period- 2 motion for appearance at $\Omega \approx 1.70872$. The unstable Hopf bifurcations of period-1 motion give the unstable saddle-node bifurcation of period-2 motions for the onset of period-2 motion. The unstable saddle-node bifurcations (USN) of period-2 motions are at $\Omega \approx 1.689$. The quantity level of $A_{(1) 1 / 2} \sim 9 \times 10^{-2}$ is observed in $\Omega \in(1.4,1.75)$. The harmonic amplitude $A_{(1) 1}$ versus rotation speed is presented in Fig. 5(iii) for the bifurcation tree of period-1 motion to period- 2 motion via the stable and unstable Hopf bifurcations. The quantity level of $A_{(1) 1}$ for the period-2 motion is in the range of $A_{(1) 1} \sim 0.5$ for $\Omega \in(1.4,1.75)$. The harmonic amplitude $A_{(1) 3 / 2}$ versus rotation speed is presented in Fig. 5(iv) with $A_{(1) 3 / 2} \sim 10^{-1}$ in $\Omega \in(1.4,1.75)$. The harmonic amplitudes $A_{(1) 2}$ and $A_{(1) 3}$ versus rotation speed are illustrated in Fig. 5(v) and (vi), respectively. Their quantity levels are $A_{(1) 2} \sim 0.3$ and $A_{(1) 3} \sim 0.16$. To reduce the abundant illustrations, the harmonic amplitudes $A_{(1) 12}$ and $A_{(1) 13}$ versus rotation speed are illustrated in Fig. 5(vii) and (viii), respectively. The quantity levels for the two harmonic amplitudes for period- 2 motions are $A_{(1) 12} \sim 3 \times 10^{-4}$ and $A_{(1) 13} \sim 2 \times 10^{-4}$.

The second branch of bifurcation tree of the period-1 motion to period- 2 motion of the nonlinear rotor in the $y$ direction is presented in Fig. 6. In Fig. 6(i), the constant term $a_{20}^{(m)}(m=1,2)$ versus rotation speed is presented. The pos- 


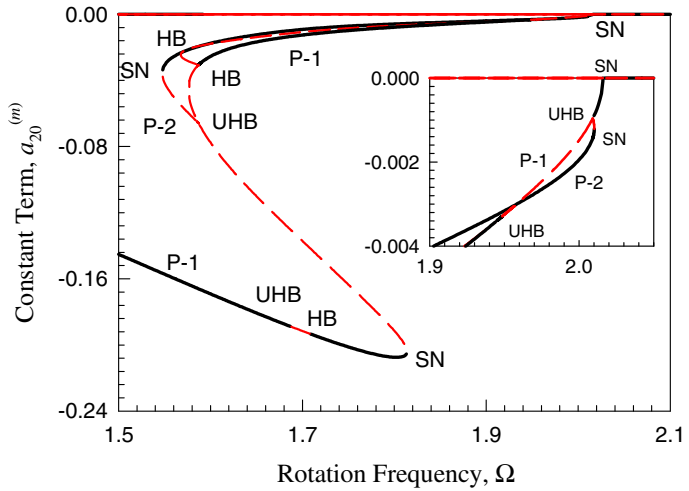

(i)

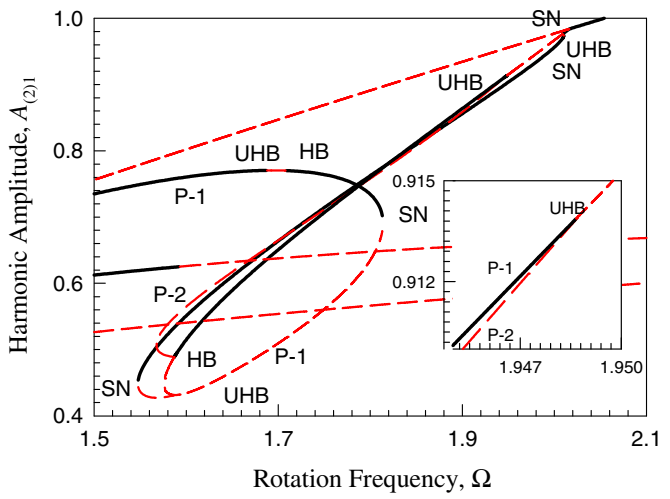

(iii)

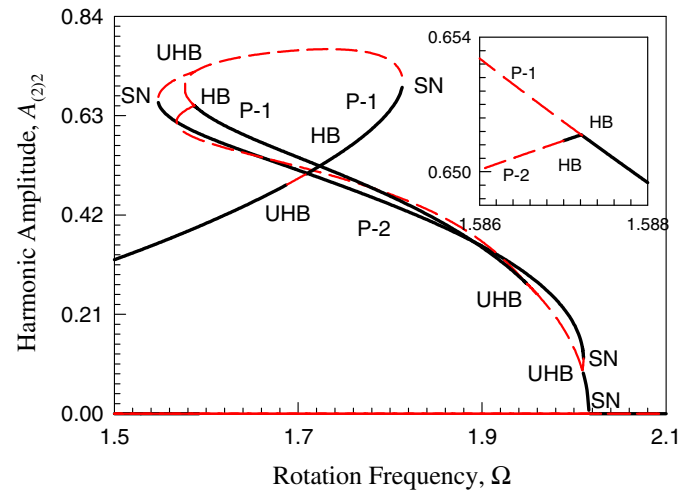

(v)

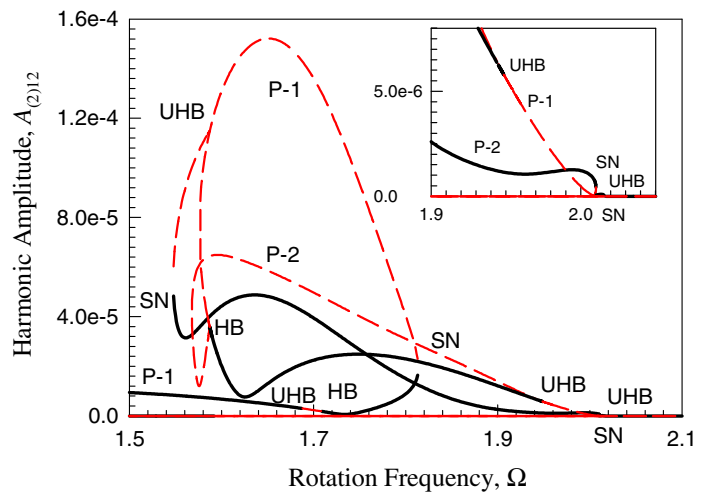

(vii)

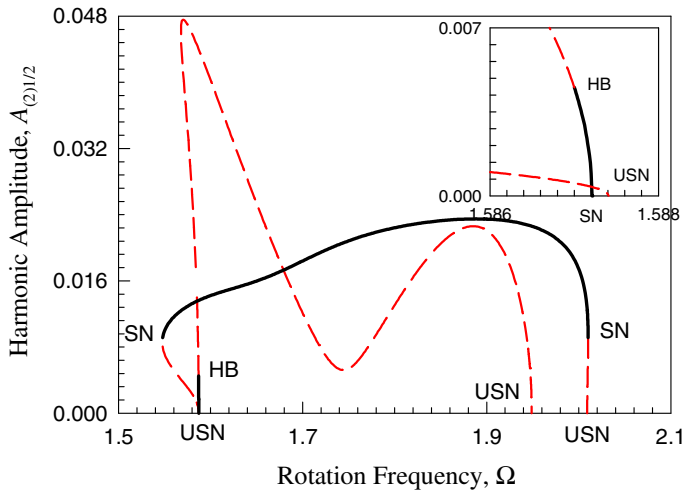

(ii)

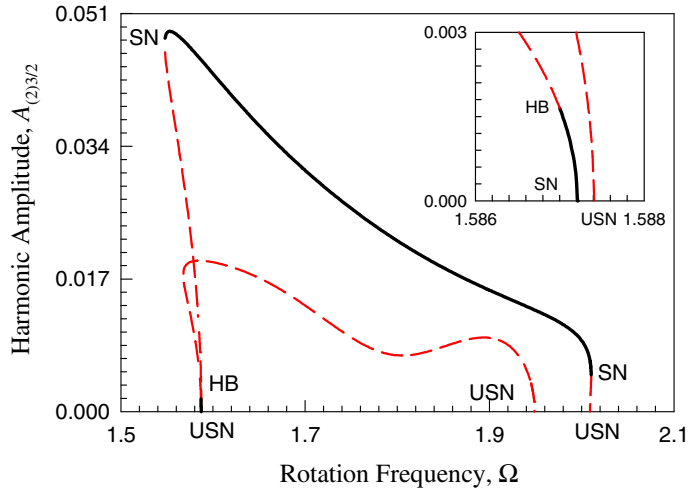

(iv)

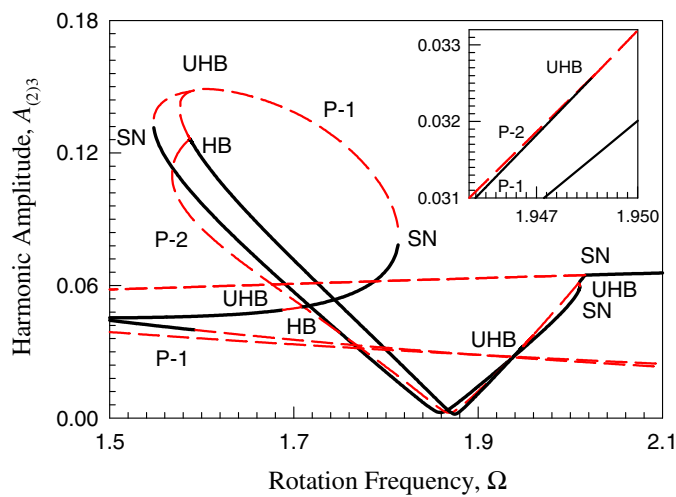

(vi)

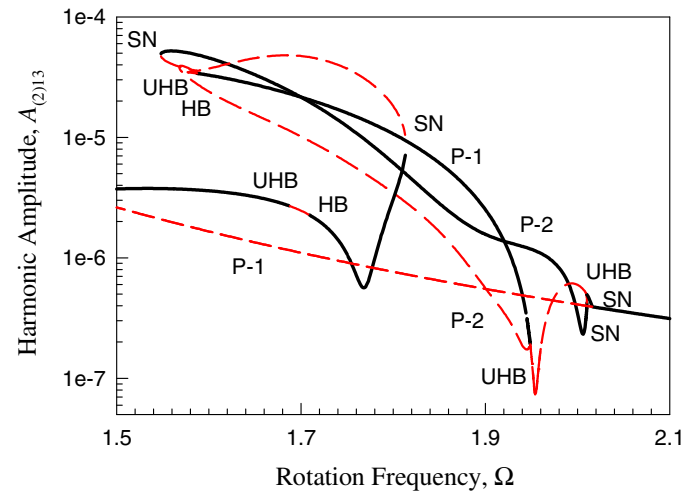

(viii)

Fig. 4 Bifurcation tree of period-1 motion to chaos of the $y$-direction in the nonlinear rotor: frequency-amplitude curves of harmonic terms based on 26 harmonic terms: (i) $a_{20}^{(m)}$, (ii)-(viii) $A_{(2) k / m}(k=1,2, \ldots, 4,6,24,26, m=2),(\delta=0.02, \alpha=0.68, \beta=10, \gamma=1.0, e=1.5)$ 


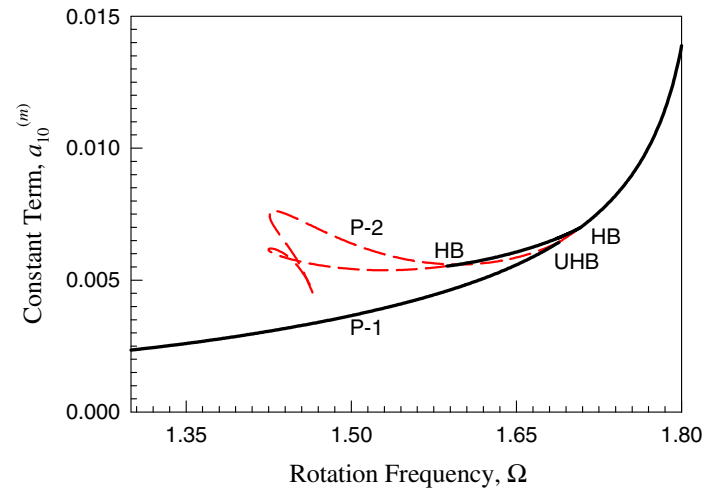

(i)

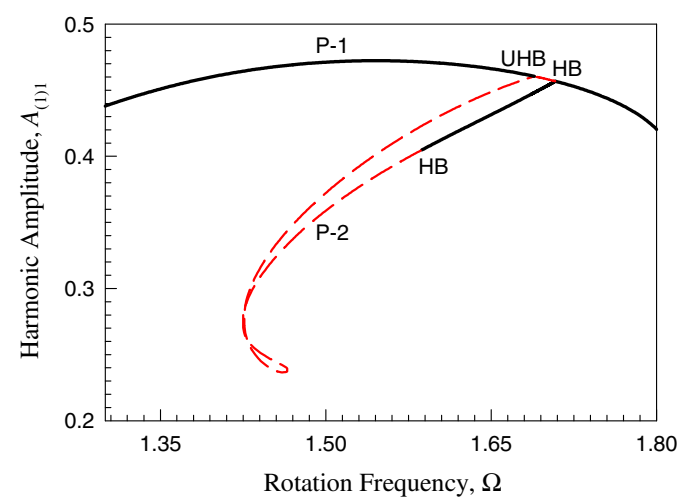

(iii)

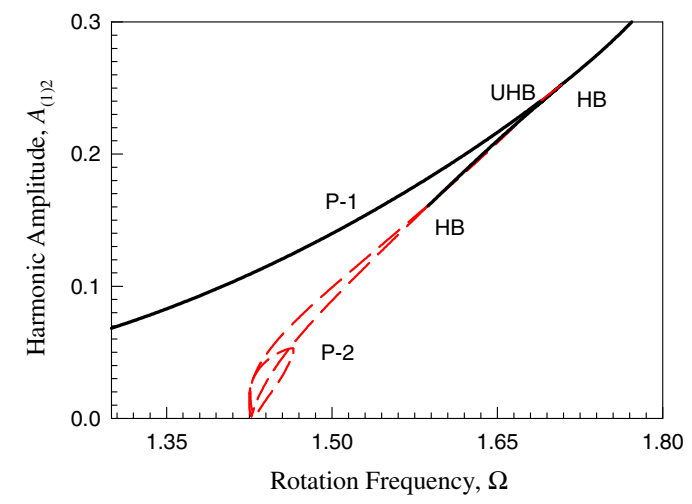

(v)

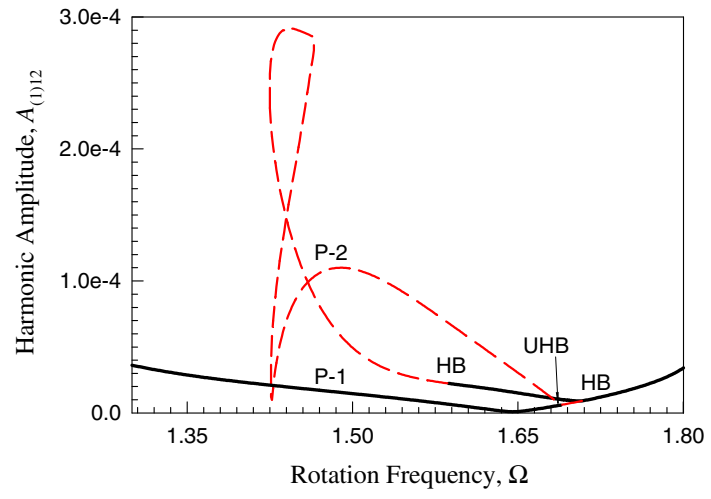

(vii)

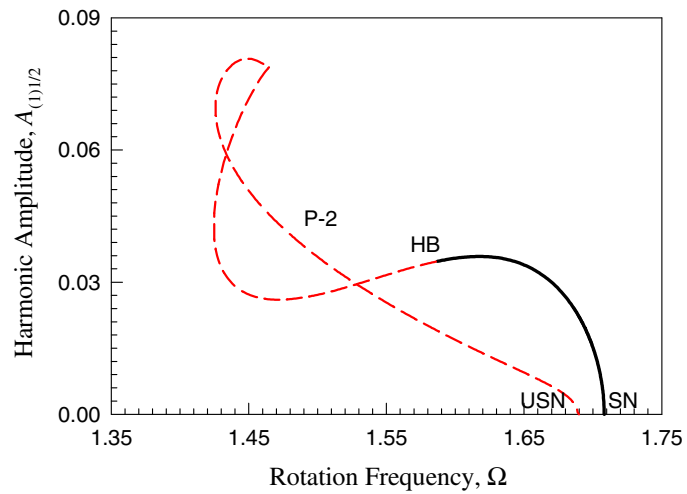

(ii)

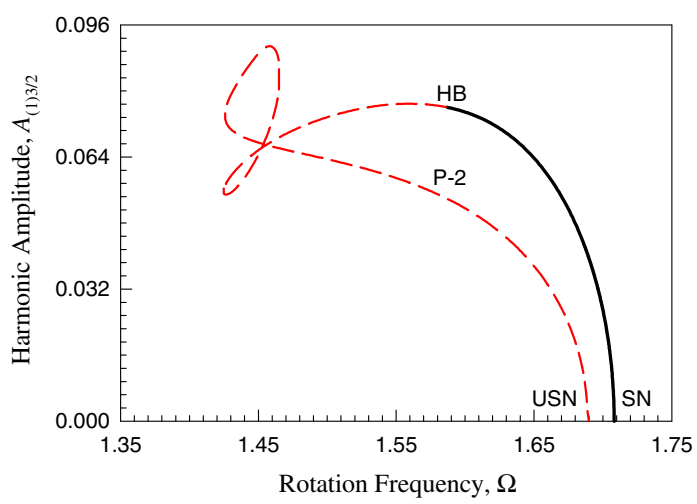

(iv)

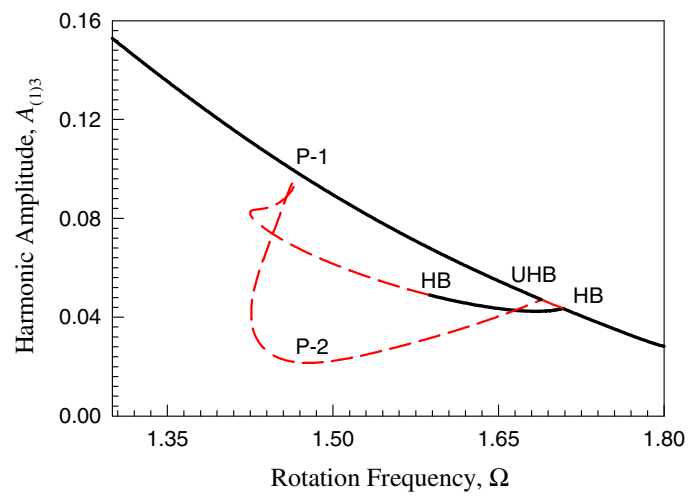

(vi)

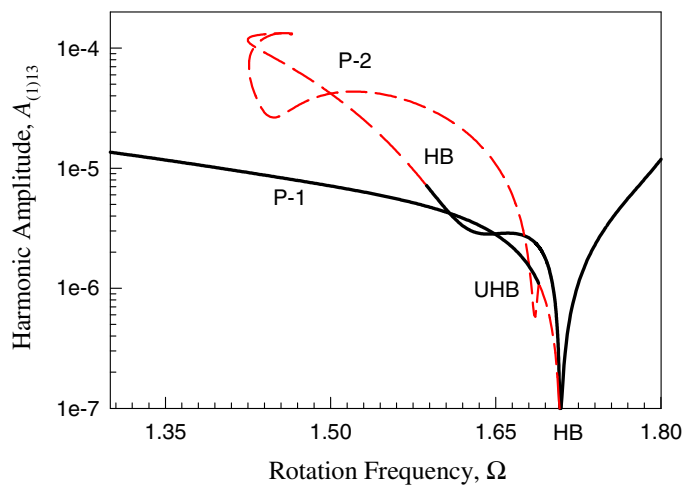

(viii)

Fig. 5 Bifurcation tree of period-1 motion to chaos of the $x$-direction in the nonlinear rotor: frequency-amplitude curves of harmonic terms based on 26 harmonic terms: (i) $a_{10}^{(m)}$, (ii)-(viii) $A_{(1) k / m}(k=1,2, \ldots, 4,6,24,26, m=2),(\delta=0.02, \alpha=0.68, \beta=10, \gamma=1.0, e=1.5)$ 


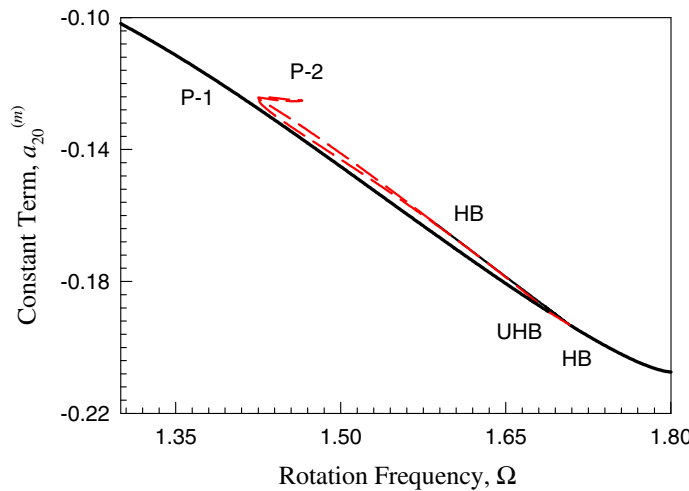

(i)

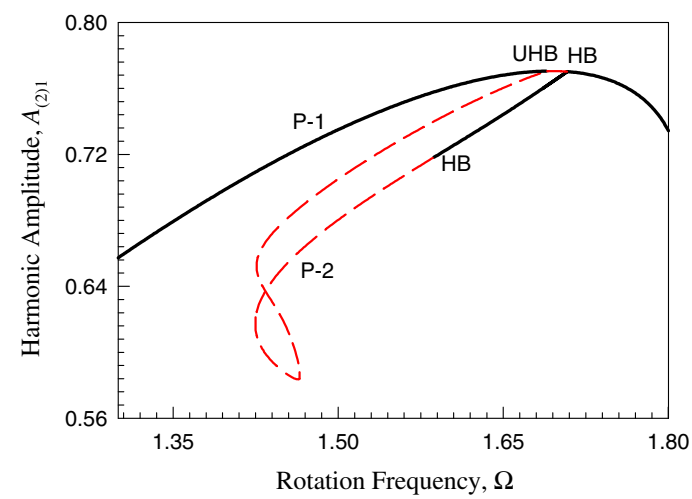

(iii)

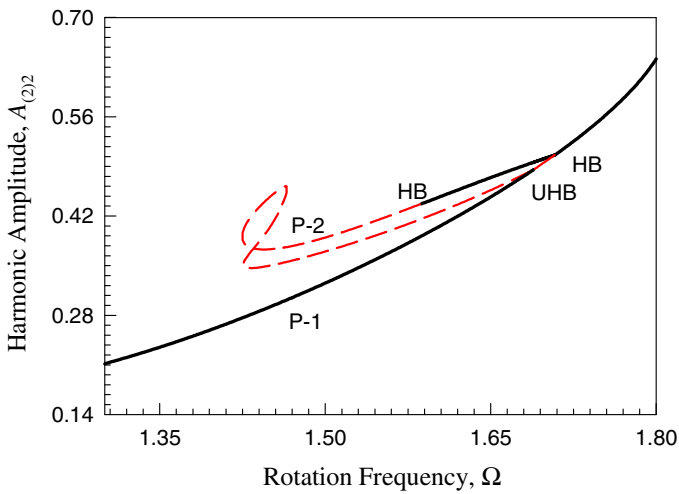

(v)

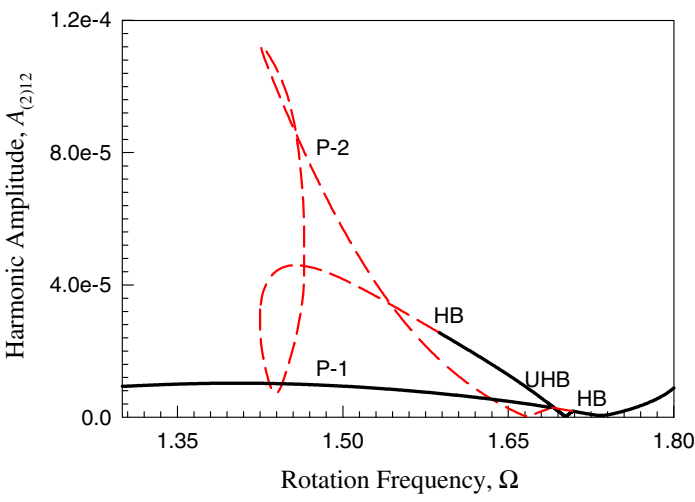

(vii)

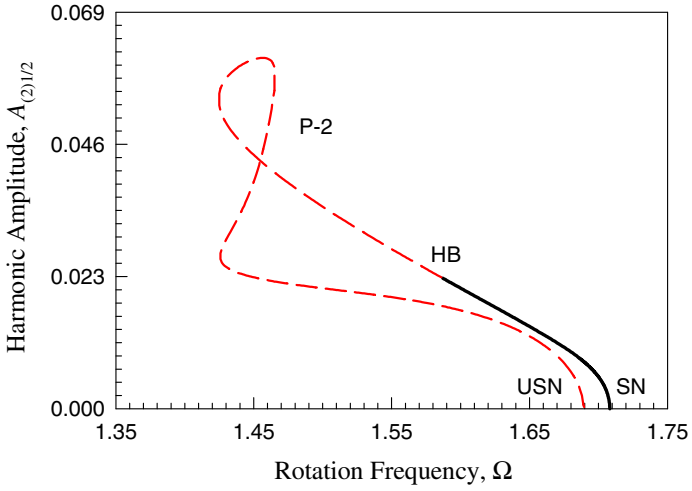

(ii)

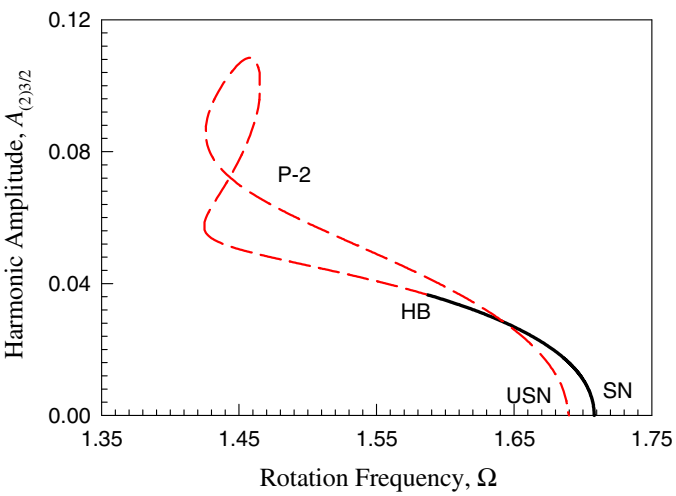

(iv)

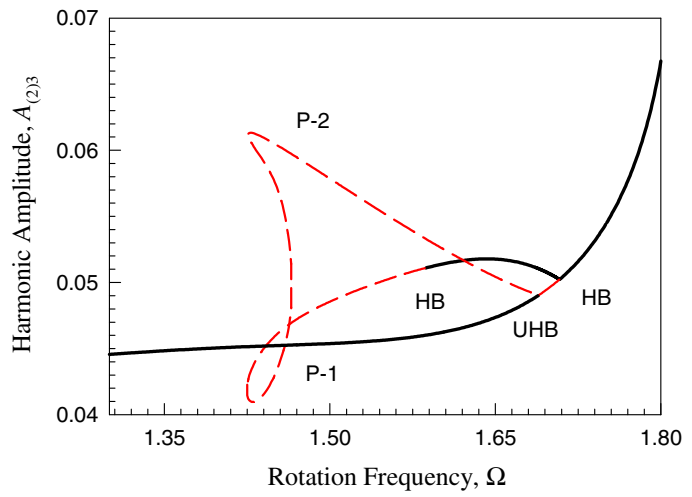

(vi)

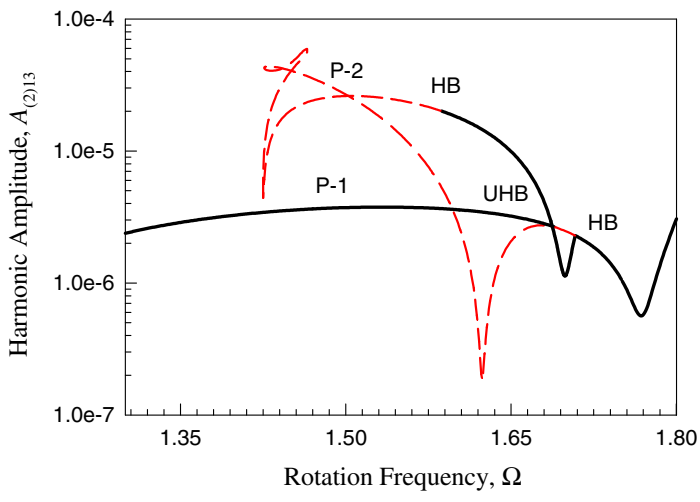

(viii)

Fig. 6 Bifurcation tree of period-1 motion to chaos of the $x$-direction in the nonlinear rotor: frequency-amplitude curves of harmonic terms based on 26 harmonic terms: (i) $a_{20}^{(m)}$, (ii)-(viii) $A_{(2) k / m}(k=1,2, \ldots, 4,6,24,26, m=2),(\delta=0.02, \alpha=0.68, \beta=10, \gamma=1.0, e=1.5)$ 


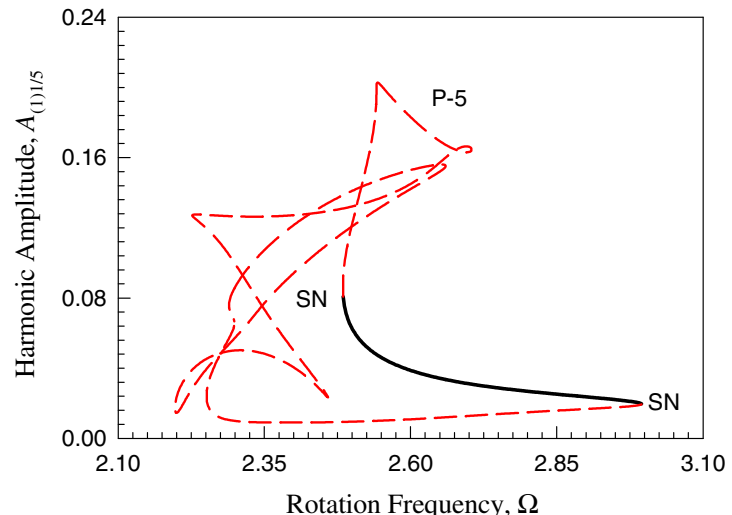

(i)

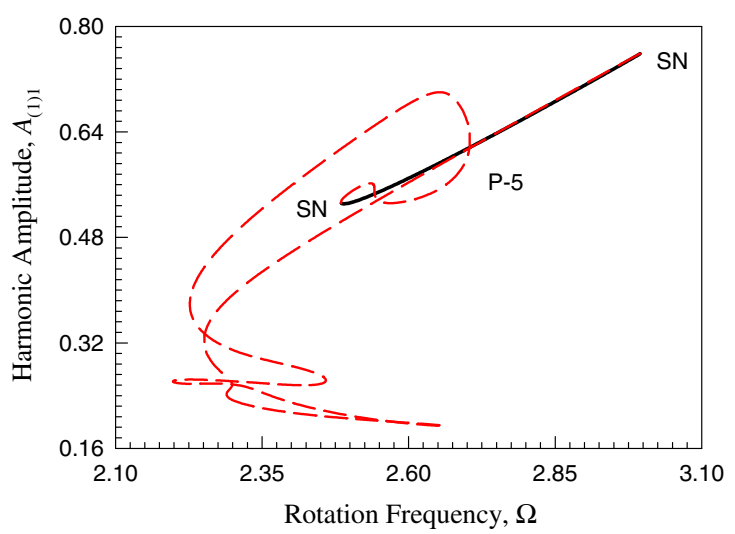

(iii)

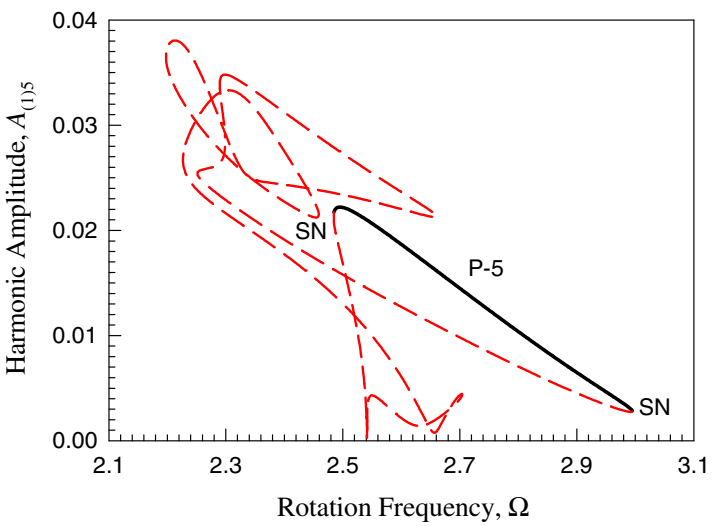

(v)

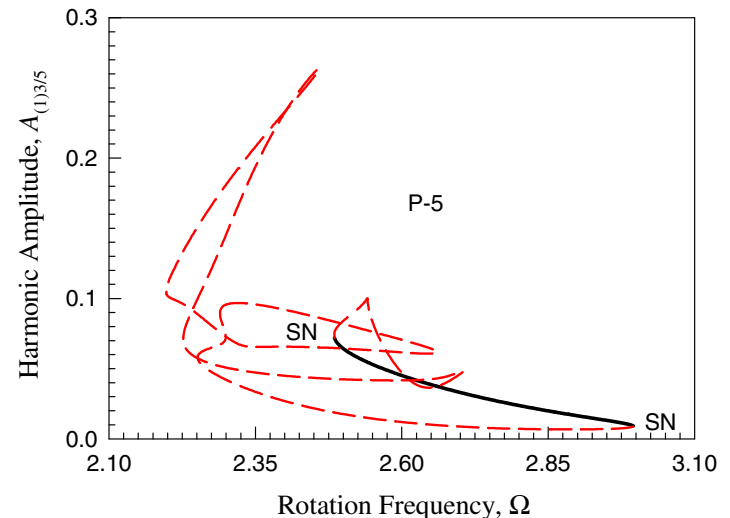

(ii)

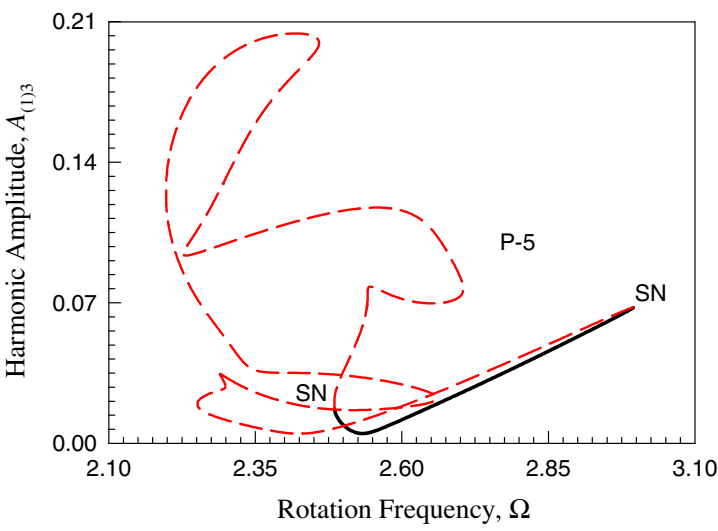

(iv)

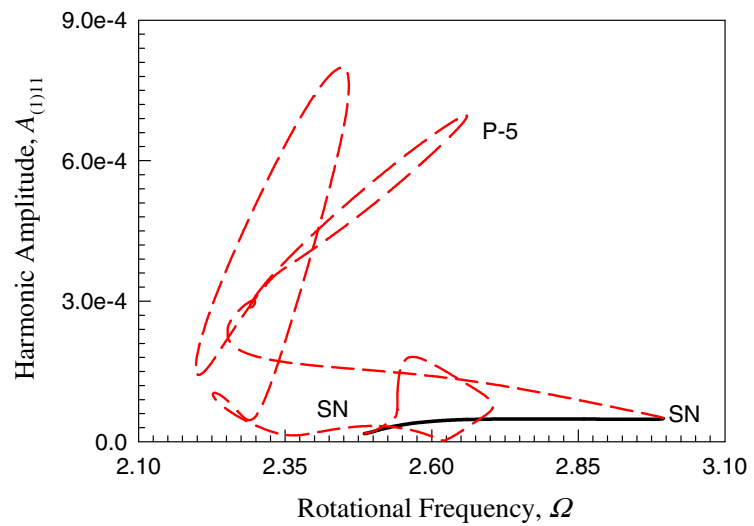

(vi)

Fig. 7 Independent period-5 motion in the $x$-direction of the nonlinear rotor: frequency-amplitude curves of harmonic terms based on 55 harmonic terms (HB55): (i)-(vi) $A_{(1) k / m}(k=1,3,5,10,15,55, m=2),(\delta=0.02 \alpha=0.68, \beta=10, \gamma=1.0, e=1.5)$

itive constant $a_{20}^{(m)}$ lies in the range of $a_{20}^{(m)} \in(-0.2,-0.1)$. The bifurcation relation of period-1 to period- 2 motion is clearly illustrated. For period-2 motion, the harmonic amplitude $A_{(2) 1 / 2}$ in the $y$-direction is presented in Fig. 6(ii), The quantity level of $A_{(2) 1 / 2} \sim 7 \times 10^{-2}$ is observed in $\Omega \in(1.4,1.75)$. The harmonic amplitude $A_{(2) 1}$ varying with rotation speed is presented in Fig. 6(iii) for the bifurcation tree of period-1 motion to period-2 motion. The quantity level of $A_{(2) 1} \sim 0.8$ for period-2 motion is observed for $\Omega \in(1.5,2.1)$. The harmonic amplitude $A_{(2) 3 / 2}$ versus rotation speed is presented in Fig. 6(iv) with $A_{(2) 3 / 2} \sim 0.12$. The harmonic amplitudes $A_{(2) 2}$ and $A_{(2) 3}$ varying with rotation speed are presented in Fig. 6(v) and (vi), respectively. The quantity levels of $A_{(2) 2} \sim 1$ and $A_{(2) 3} \sim 0.07$ are observed. To reduce abundant illustrations, the harmonic amplitudes $A_{(1) 12}$ and $A_{(1) 13}$ versus rotation speed are illustrated in Fig. 6(vii) and (viii), respectively. The quantity levels for the two harmonic ampli- 


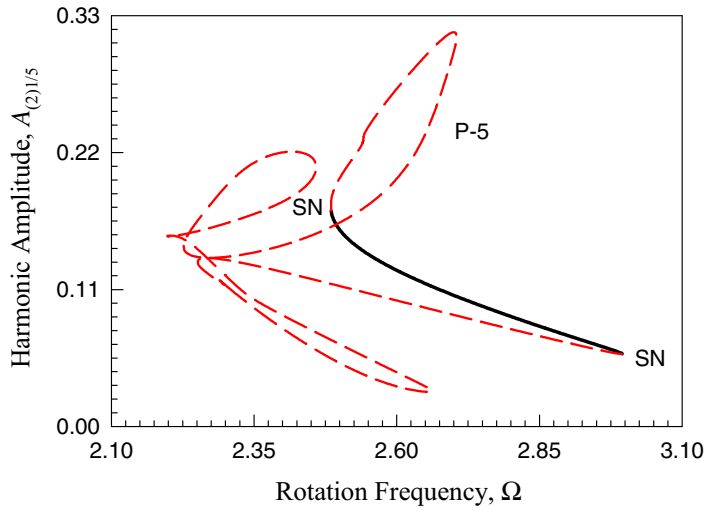

(i)

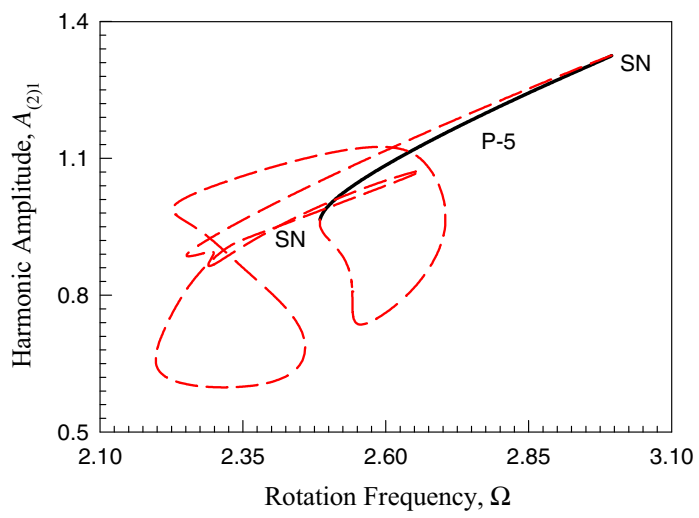

(iii)

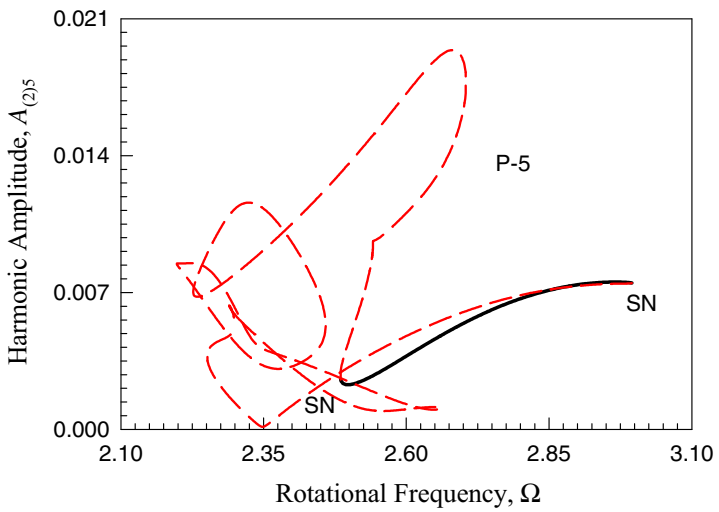

(v)

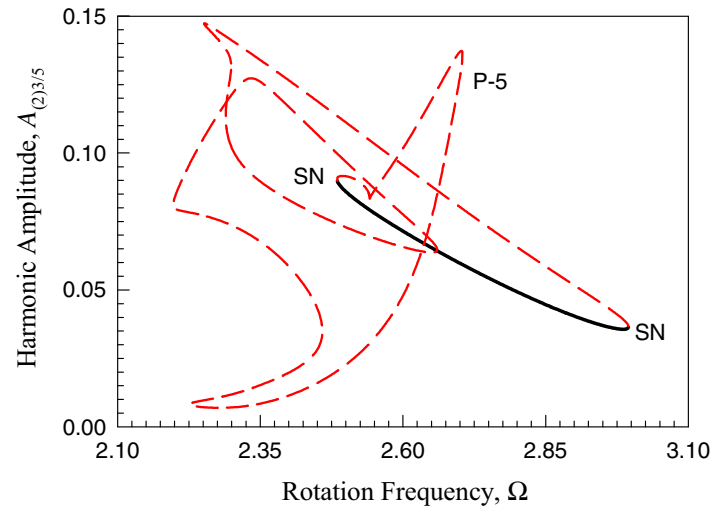

(ii)

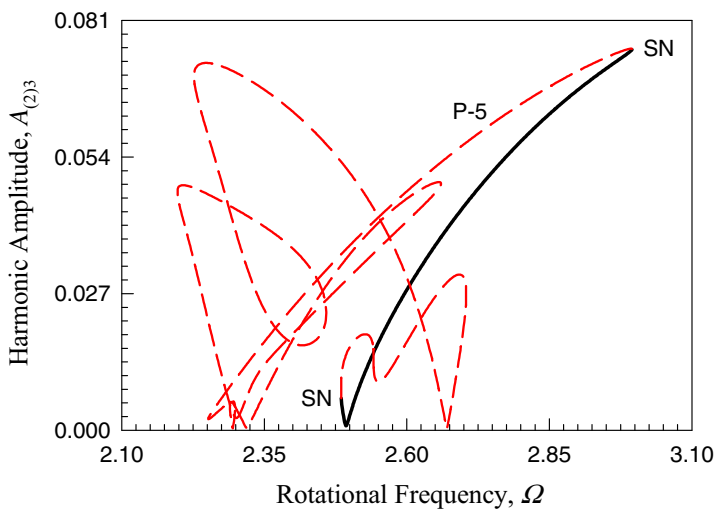

(iv)

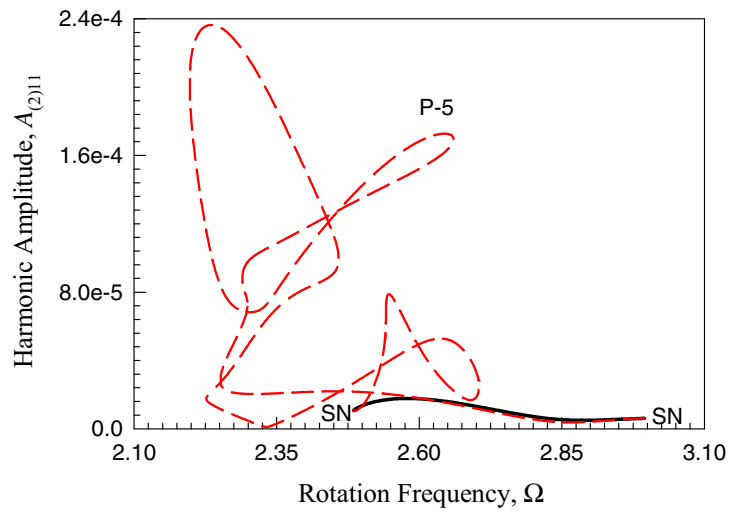

(vi)

Fig. 8 Independent period-5 motion in the $y$-direction of the nonlinear rotor: frequency-amplitude curves of harmonic terms based on 55 harmonic terms (HB55): (i)-(vi) $A_{(1) k / m}(k=1,3,5,10,15,55, m=2),(\delta=0.02 \alpha=0.68, \beta=10, \gamma=1.0, e=1.5)$

tudes for period-2 motions are $A_{(2) 12} \sim 1.2 \times 10^{-4}$ and $A_{(2) 13} \sim 1 \times 10^{-4}$.

\subsection{Independent period-5 motion}

In the previous section, the period-1 motion to period-2 motions is presented via bifurcations. Herein, the analytical solution of an independent period- 5 motion is based on 55 harmonic terms, and the frequency-amplitude characteris- tics are presented in Figs. 7 and 8. This independent period-5 motion is symmetric with saddle-node bifurcations only.

In Fig. 7, the frequency-amplitude characteristics of period-5 motion for the $x$-direction of the nonlinear rotor are presented. Since the independent period-5 motion is symmetric, $a_{10}^{(5)}=0$ and $A_{(1) k / m}=0(k=2 l, \quad l=1,2, \ldots$ and $m=5)$ is obtained. Thus, only $A_{(1) k / m}(k=2 l+1, \quad l=$ $1,2, \ldots$ and $m=5$ ) are presented. In Fig. 7(i), the harmonic amplitude $A_{(1) 1 / 5}$ versus rotation speed $\Omega$ is presented. The two saddle-node bifurcations occur at $\Omega=2.485,2.995$. 


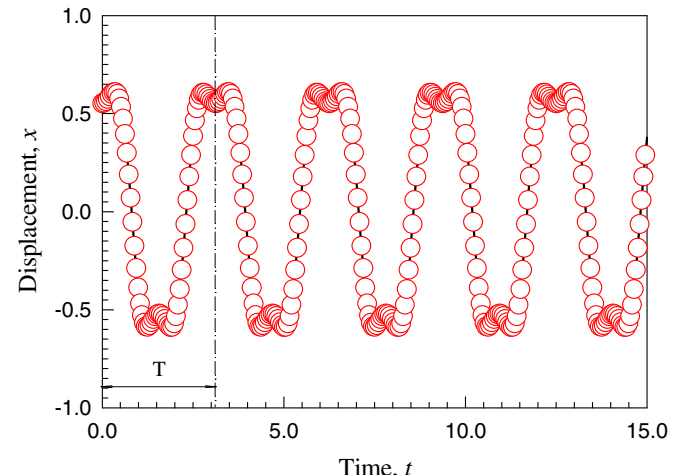

(i)

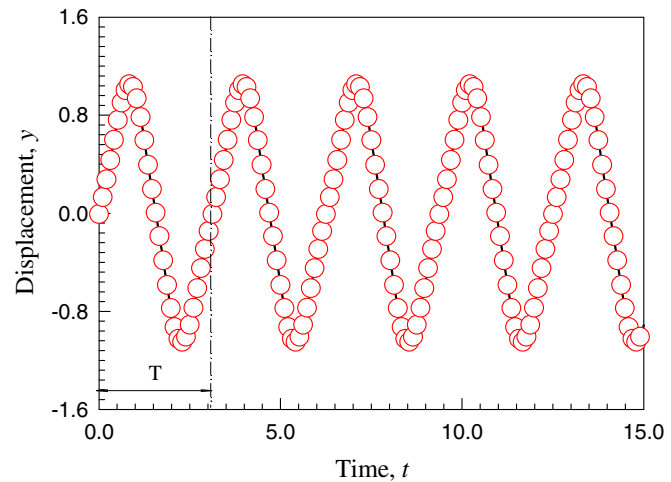

(iii)

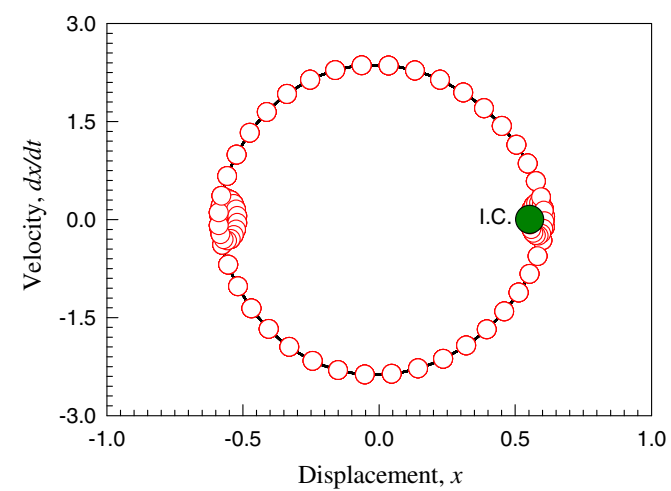

(v)

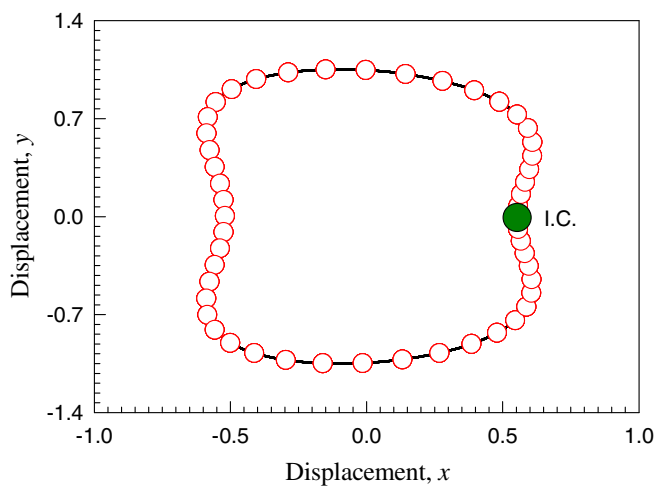

(vii)

Fig. 9 Period-1 motion of a nonlinear rotor ( $\Omega=2.01$, HB13): (i) $x$-displacement, (ii) $x$-velocity; (iii) $y$-displacement, (iv) $y$ velocity; (v) $x$-trajectory and (vi) $y$-trajectory; (vii) displacement orbit, (viii) $x$-harmonic amplitude, and (ix) $y$-harmonic amplitude.

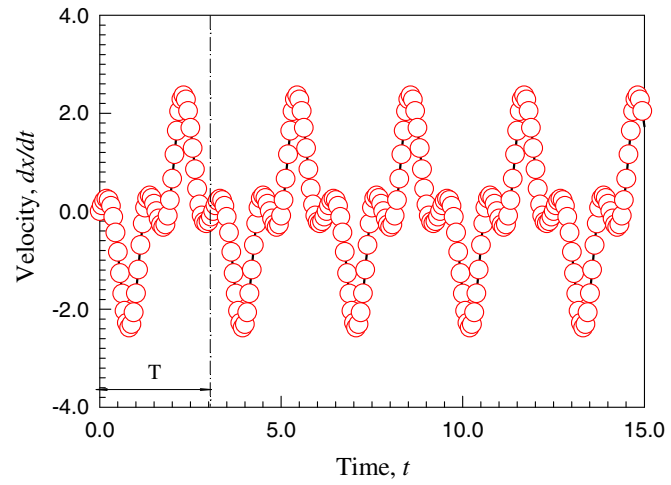

(ii)

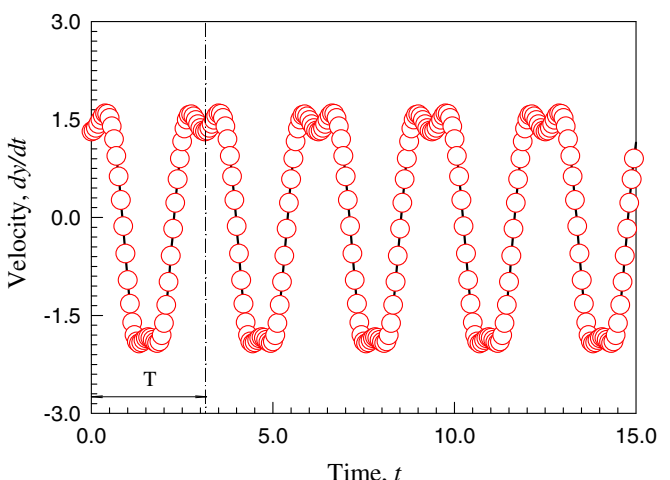

(iv)

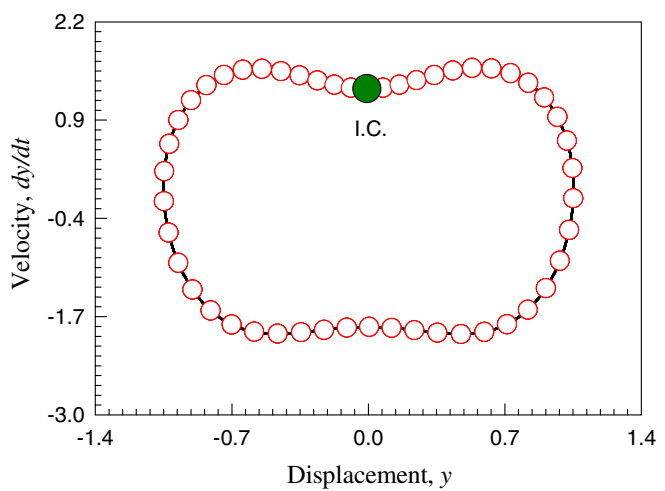

(vi)

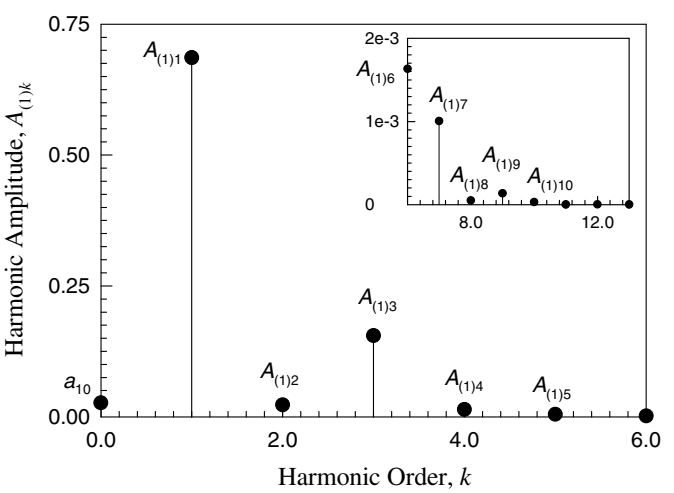

(viii)

Initial condition $\left(x_{0}, \dot{x}_{0}\right)=(0.552759,2.309360 \mathrm{E}-3)$ and $\left(y_{0}, \dot{y}_{0}\right)=$ $(-7.387180 \mathrm{E}-3,1.315090) .(\delta=0.02, \alpha=0.68, \beta=10, \gamma=$ $1.0, e=1.5)$ 


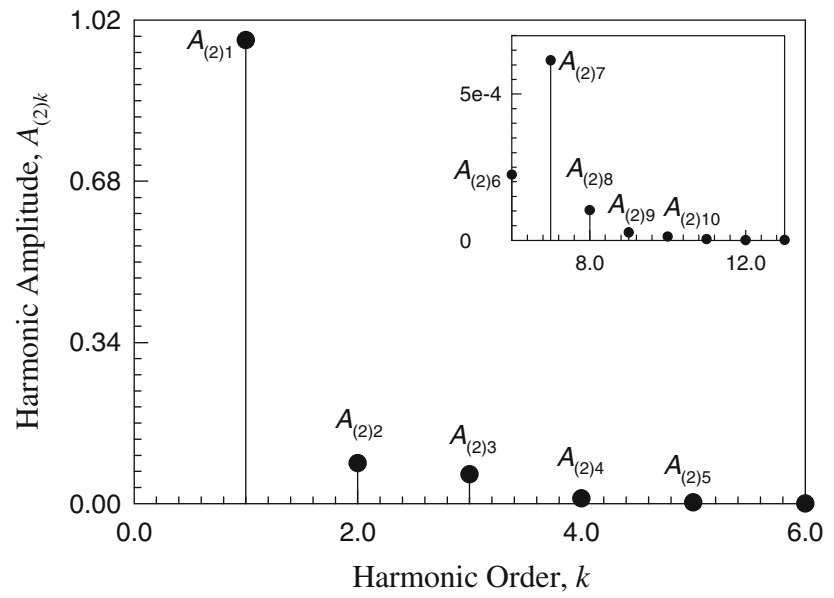

(ix)

Fig. 9 continued

The stable and unstable period-5 motions form a closed loop in the frequency-amplitude curve. The quantity levels of stable and unstable period-5 motions are $A_{(1) 1 / 5} \sim 0.08$ and $A_{(1) 1 / 5} \sim 0.24$, respectively. The harmonic amplitude $A_{(1) 3 / 5}$ varying with rotation speed $\Omega$ is presented in Fig. 7(ii). The quantity levels of $A_{(1) 1 / 5}$ and $A_{(1) 3 / 5}$ are quite similar. The primary harmonic amplitude $A_{(1) 1}$ versus rotation speed is arranged in Fig. 7(iii). The quantity level of $A_{(1) 1}$ is very large with $A_{(1) 1} \sim 1$. To reduce abundant illustrations, the harmonic amplitudes $A_{(1) 3}$ and $A_{(1) 5}$ are presented in Fig. 7(iv), (v), respectively. The corresponding quantity levels of the harmonic amplitudes are $A_{(1) 3} \sim 0.2$ and $A_{(1) 5} \sim$ 0.04 , respectively. The 11th harmonic amplitude varying with rotation speed is presented in Fig. 7(vi). The quantity levels of harmonic amplitudes for the stable and unstable period-5 motions are $A_{(1) 11} \sim 10^{-4}$ and $A_{(1) 11} \sim 10^{-3}$, respectively.

In Fig. 8, the frequency-amplitude characteristic of period5 motion for the $y$-direction of the nonlinear rotor is also presented. In Fig. 8(i), the harmonic amplitude $A_{(2) 1 / 5}$ versus rotation speed $\Omega$ is presented. The quantity levels of stable and unstable period-5 motions are $A_{(2) 1 / 5} \sim 0.2$ and $A_{(2) 1 / 5} \sim 0.3$, respectively. The harmonic amplitude $A_{(2) 3 / 5}$ varying with rotation speed $\Omega$ is presented in Fig. 8(ii). The quantity level of $A_{(2) 3 / 5}$ reduces to $A_{(2) 3 / 5} \sim 0.15$. The primary harmonic amplitude $A_{(2) 1}$ versus rotation speed is arranged in Fig. 8(iii). The quantity level of $A_{(2) 1}$ is very large with $A_{(2) 1} \sim 1.5$. To reduce abundant illustrations, the harmonic amplitudes $A_{(2) 3}$ and $A_{(2) 5}$ are presented in Fig. 8(iv), (v), respectively. The corresponding quantity levels of the harmonic amplitudes are $A_{(2) 3} \sim 0.08$ and $A_{(2) 5} \sim 0.02$, respectively. The 11 th harmonic amplitude varying with rotation speed is presented in Fig. 8(vi). The quantity levels of harmonic amplitudes for the stable and unstable period-5 motions are $A_{(2) 11} \sim 2 \times 10^{-5}$ and $A_{(2) 11} \sim 2.4 \times 10^{-4}$, respectively.

\section{Numerical illustrations}

To illustrate period-m motions in the nonlinear rotor system, numerical and analytical solutions will be presented. The initial conditions for numerical simulations are computed from approximate analytical solutions of periodic solutions. In all plots, circular symbols gives approximate solutions, and solid curves give numerical simulation results. The acronym "I.C." with a large circular symbol represents initial condition for all plots. The numerical solutions of periodic motions are generated via the midpoint discrete scheme.

In Fig. 9, a period-1 motion based on 13 harmonic terms (HB13) is presented for $\Omega=2.01$ with other parameters in Eq. (54). The displacement and velocity responses in the $x$-direction of the nonlinear rotor are presented in Fig. 9(i), (ii), respectively. One period $(T)$ for the period-1 motion response is labeled in the two plots. Similarly, the displacement and velocity responses in the $y$-direction of the nonlinear rotor are also presented in Fig. 9(iii), (iv), respectively. The analytical and numerical solutions match very well. The two trajectories for $x$ and $y$-directions are presented for over 40 periods in Fig. 9(v), (vi), respectively. the two trajectories are different because of the interaction. The initial conditions are marked by large circular symbols and labeled by "I.C.". In engineering, one is interested in displacement orbits in rotor dynamics. The displacement orbit of rotor in $x$ and $y$-directions is presented in Fig. 9(vii). For better understanding of harmonic contributions, the harmonic amplitude spectrums of rotor in $x$ and $y$-directions are presented in Fig. 9(viii), (ix). The harmonic amplitude spectrums are computed from analytical solutions. The main harmonic amplitudes of rotor in the $x$-direction are $a_{10} \approx 0.026626, A_{(1) 1} \approx$ $0.685920, A_{(1) 2} \approx 0.022759, A_{(1) 3} \approx 0.155060$, and $A_{(1) 4} \approx$ 0.014091 . The other harmonic amplitudes in the $x$-direction are $A_{(1) 5} \sim 5 \times 10^{-3}, A_{(1) k} \sim 10^{-3}(k=6,7), A_{(1) 8} \sim 5 \times$ $10^{-5}, \quad A_{(1) 9} \sim 10^{-4}, \quad A_{(1) 10} \sim 3 \times 10^{-5}, A_{(1) k} \sim 10^{-6}$ $(k=11,12,13)$. However, the main harmonic amplitudes of rotor in the $y$-direction are $a_{20} \approx 8.97 \times 10^{-4}, \quad A_{(2) 1} \approx$ $0.977544, A_{(2) 2} \approx 0.085401, A_{(2) 3} \approx 0.061716$, and $A_{(2) 4} \approx 0.010812$. The other harmonic amplitudes in the $y$-direction are $A_{(2) 5} \sim 2.5 \times 10^{-3}, A_{(1) k} \sim 10^{-4}(k=$ 6, 8), $A_{(2) 7} \sim 6 \times 10^{-4}, \quad A_{(2) 9} \sim 3 \times 10^{-5}, \quad A_{(2) 10} \sim$ $10^{-5}, A_{(2) 11} \sim 3 \times 10^{-6}, \quad A_{(2) 12} \sim 5 \times 10^{-8}$, and $A_{(2) 13} \sim 5 \times 10^{-7}$.

In Fig. 10, a period-2 motion based on 26 harmonic terms (HB26) are presented for $\Omega=2.01$ with other parameters in Eq. (54). The time-histories of displacement and velocity in the $x$-direction of the nonlinear rotor are presented in Fig. 10(i), (ii), respectively. Compared to the coexisting 


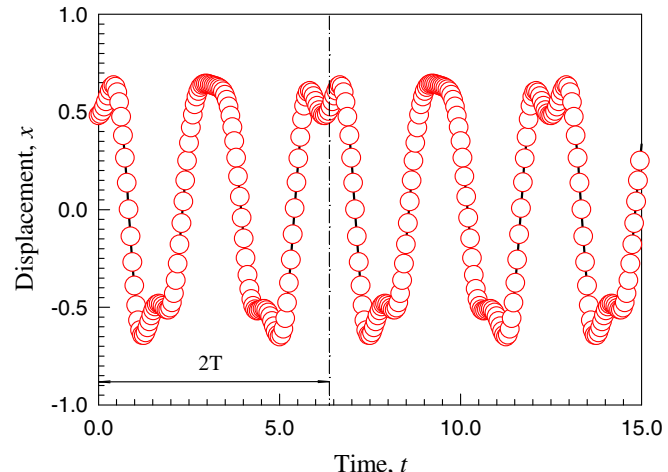

(i)

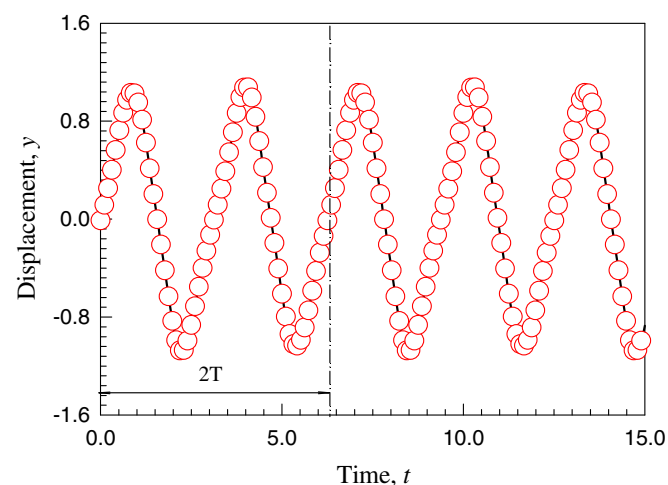

(iii)

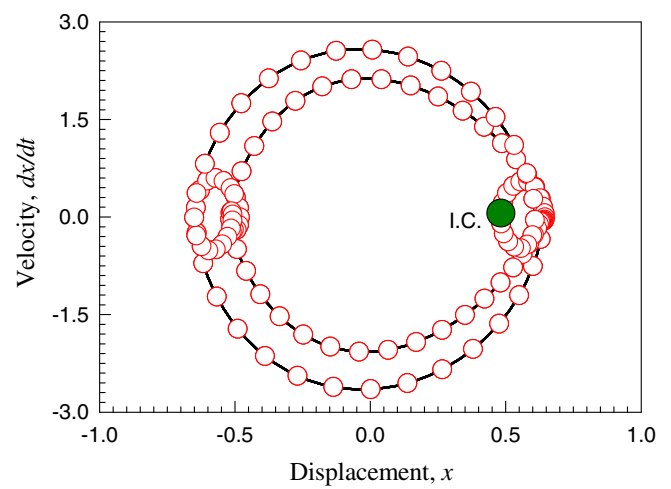

(v)

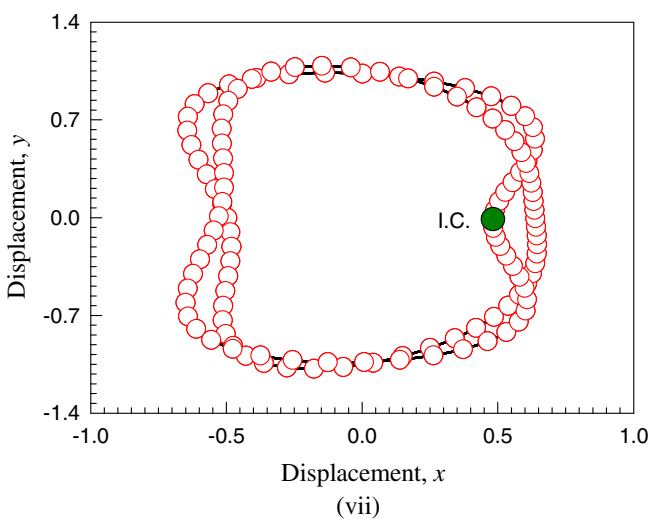

Fig. 10 Stable period-2 motion of a nonlinear rotor $(\Omega=2.01$, HB26): (i) $x$-displacement, (ii) $x$-velocity; (iii) $y$-displacement, (v) $y$-velocity; (v) $x$-trajectory and (vi) $y$-trajectory; (vii) displacement orbit, (viii) $x$-harmonic amplitude, and (ix) $y$-harmonic amplitude.

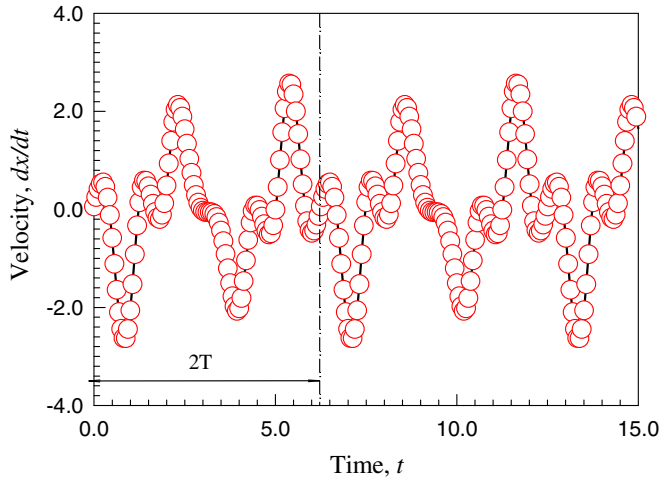

(ii)

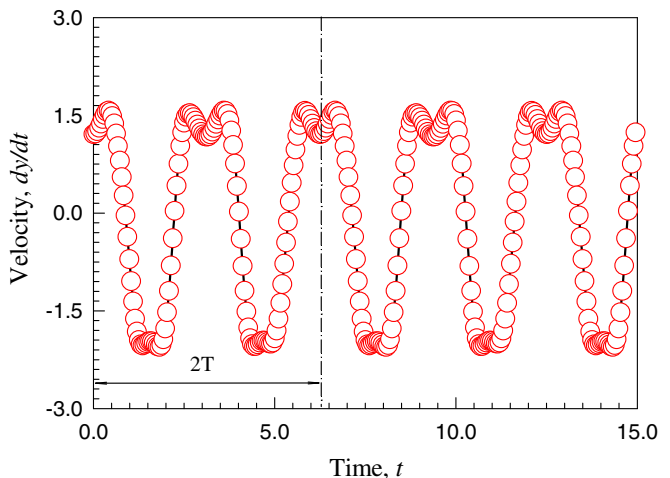

(iv)

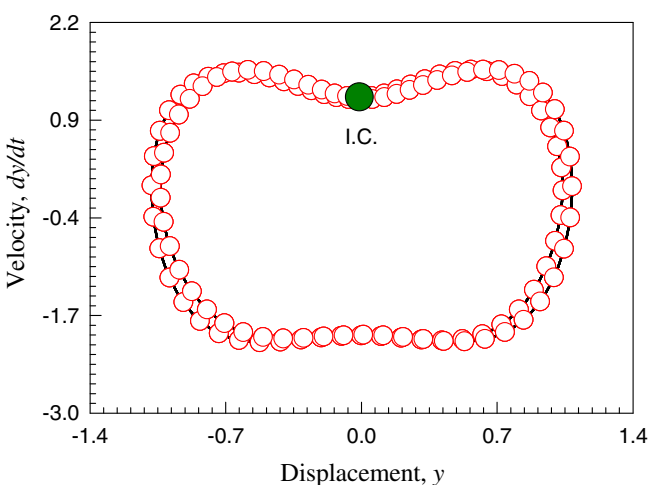

(vi)

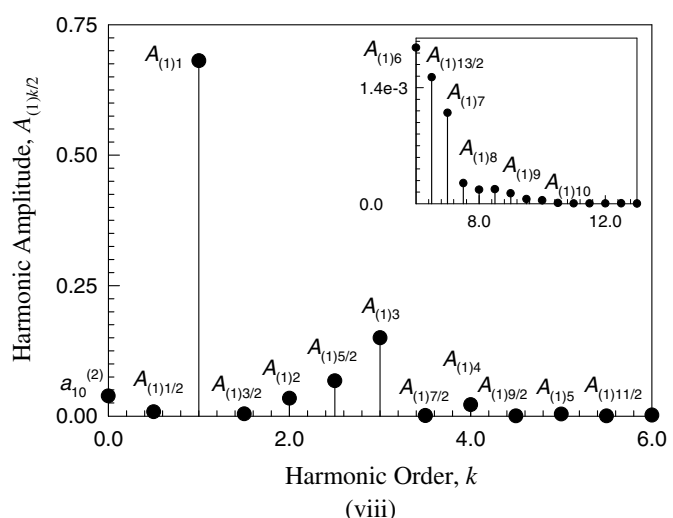

Initial conditions $\left(x_{0}, \dot{x}_{0}\right)=(0.482128,0.059237)$ and $\left(y_{0}, \dot{y}_{0}\right)=$ $(-0.010820,1.208560) .(\delta=0.02, \alpha=0.68, \beta=10, \gamma=1.0, e=$ $1.5)$ 


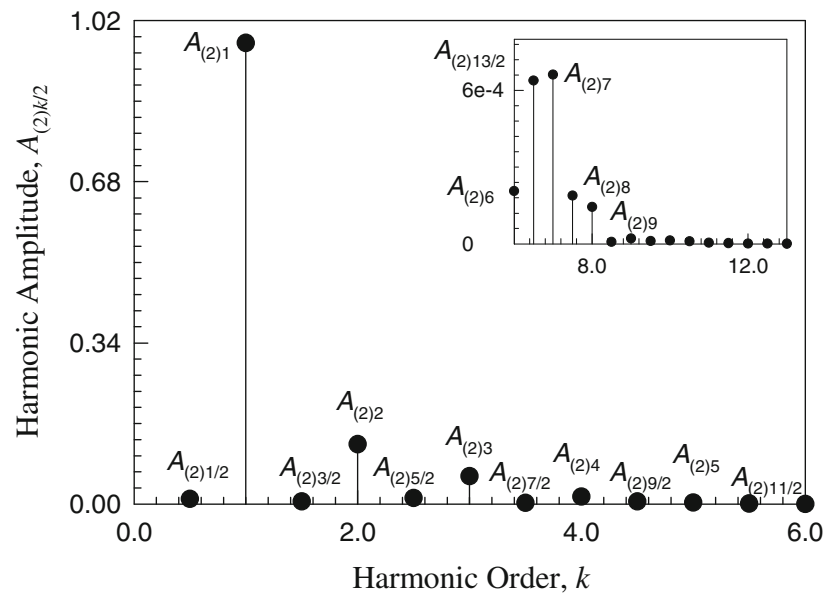

(ix)

Fig. 10 continued

period-1 motion, the displacement and velocity of period2 motion cannot keep the period-1 motion patterns. Two periods $(2 T)$ for the period- 2 motion is labeled in the two plots. The time-histories of displacement and velocity in the $y$-direction of the nonlinear rotor are also presented in Fig. 10(iii), (iv), respectively. The two trajectories for $x$ and $y$-directions are presented for over 40 periods in Fig. 10(v), (vi), respectively. Compared to period-1 motion, the period-doubling responses are clearly observed. The initial conditions are marked by large circular symbols and also labeled by "I.C.". The displacement orbit of rotor in the $x$ and $y$-directions is presented in Fig. 10(vii). To show harmonic contributions on the period- 2 motion, the harmonic amplitude spectrums of the stable period-2 motion of the nonlinear rotor in the $x$ and $y$-directions are presented in Fig. 10(viii), (ix). The harmonic amplitude spectrums of the stable period-2 motion are given by analytical solutions. The main harmonic amplitudes in the $x$-direction are $a_{10}^{(2)} \approx 0.038564, A_{(1) 1} \approx 0.680973, A_{(1) 2} \approx$ $0.034070, A_{(1) 3} \approx 0.149972$, and $A_{(1) 4} \approx 0.021612$. However, $A_{(1) 1 / 2} \approx 8.34 \times 10^{-3}, \quad A_{(1) 3 / 2} \approx 4.04 \times$ $10^{-3}, \quad A_{(1) 5 / 2} \approx 0.067610, A_{(1) 7 / 2} \approx 9.36 \times 10^{-4}$. The other harmonic amplitudes in the $x$-direction are $A_{(1) 9 / 2} \sim 2 \times 10^{-4}, A_{(1) 5} \sim 4 \times 10^{-3}, A_{(1) 11 / 2} \sim$ $1.2 \times 10^{-4}, \quad A_{(1) 6} \sim 2 \times 10^{-4}, \quad A_{(1) 13 / 2} \sim 1.5 \times$ $10^{-3}, \quad A_{(1) 7} \sim 10^{-3}, A_{k / 2} \in\left(10^{-6}, 10^{-4}\right)(k=$ $16,17, \ldots 26)$. However, the main harmonic amplitudes in the $y$-direction are $a_{20}^{(2)} \approx-1.3 \times 10^{-3}, A_{(2) 1} \approx$ $0.972574, A_{(2) 2} \approx 0.126123, A_{(2) 3} \approx 0.058972$, and $A_{(2) 4} \approx 0.015588$. However, $A_{(2) 1 / 2} \approx 0.010661, A_{(2) 3 / 2}$ $\approx 5.50 \times 10^{-3}, \quad A_{(2) 5 / 2} \approx 0.012854, \quad A_{(2) 7 / 2} \approx 2.56 \times$ $10^{-4}$. The other harmonic amplitudes in the $y$-direction are $A_{(2) 9 / 2} \sim 5.39 \times 10^{-3}, A_{(2) 5} \sim 3.26 \times 10^{-3}, A_{(2) 11 / 2} \sim$ $9.65 \times 10^{-4}, A_{(2) 6} \sim 2.06 \times 10^{-4}, A_{(2) 13 / 2} \sim 6.4 \times$
$10^{-4}, \quad A_{(2) 7} \sim 6.6 \times 10^{-4}, A_{(2) k / 2} \in\left(10^{-7}, 10^{-4}\right)(k=$ $16,17, \ldots 26)$.

For rotation speed $\Omega=1.63$, a stable period-1 motion, a stable period- 2 motion and an unstable period- 2 motion coexist, as shown in Fig. 11. The input data for numerical simulation is listed in Table 1. To reduce illustrations, the time-histories of displacements and velocities in the $x$ and $y$-directions will not be presented herein. The $x$ and $y$ - trajectories, displacement orbit and, $x$ and $y$-harmonic spectrums for period-1 motion are presented in Fig. 11(i)(v) for $\Omega=1.63$, respectively. The analytical solutions are based on 13 harmonic terms (HB13). Two trajectories of the $x$ and $y$-directions of the nonlinear rotor possess a simple period- 1 cycle. The analytical and numerical results match very well. The main harmonic amplitudes of the stable period-1 motion in the $x$-direction are $a_{10}^{(2)} \approx 5.22 \times$ $10^{-3}, A_{(1) 1} \approx 0.468400, A_{(1) 2} \approx 0.204974, A_{(1) 3} \approx$ $0.058732, A_{(1) 4} \approx 0.056108$, and $A_{(1) 5} \approx 0.021847$. The other harmonic amplitudes of the stable period-1 motion in the $x$-direction are $A_{(1) k} \in\left(10^{-6}, 10^{-3}\right)(k=6,7, \ldots, 13)$. However, the main harmonic amplitudes of the stable period1 motion in the $y$-direction are $a_{20}^{(2)} \approx-0.176100, A_{(2) 1} \approx$ $0.765510, \quad A_{(2) 2} \approx 0.428596, A_{(2) 3} \approx 0.046782, A_{(2) 4}$ $\approx 0.035956$, and $A_{(2) 5} \approx 0.017391$. The other harmonic amplitudes in the $y$-direction are $A_{(2) k} \in\left(10^{-6}, 10^{-3}\right)(k=$ $6,7, \ldots, 13)$.

The $x$ and $y$ - trajectories, displacement orbit and, the $x$ and $y$-harmonic spectrums for stable period-2 motion are presented in Fig. 11(vi)-(x) for $\Omega=1.63$, respectively. The analytical solutions based on 26 harmonic terms (HB26). The two trajectories for period-2 motion do not simply repeat the trajectories of the period-1 motion. The displacement orbit becomes very complicated, as shown in Fig. 11(viii). The analytical and numerical results are in a good agreement. The $x$ and $y$ - harmonic spectrums of the stable period-2 motion are presented in Fig. 11(ix), (x). The main harmonic amplitudes of the stable period-2 motion in the $x$-direction are $a_{10}^{(2)} \approx 5.85 \times 10^{-3}, A_{(1) 1} \approx$ $0.423200, A_{(1) 2} \approx 0.194718, A_{(1) 3} \approx 0.044920, A_{(1) 4} \approx$ 0.053038 , and $A_{(1) 5} \approx 0.028407$. However, $A_{(1) 1 / 2} \approx$ $0.035560, A_{(1) 3 / 2} \approx 0.069786, A_{(1) 5 / 2} \approx 0.185101$, $A_{(1) 7 / 2} \approx 0.062456$, and $A_{(1) 9 / 2} \approx 5.93 \times 10^{-4}$. Compared to the period-2 motion presented before, the effects of harmonic amplitudes $A_{(1) 2 l / 2}(l=1,2, \ldots)$ on the period-2 motion becomes large. The other harmonic amplitudes in the $x$-direction are $A_{(1) k / 2} \in\left(10^{-6}, 10^{-3}\right)(k=16,17, \ldots 26)$. However, the main harmonic amplitudes of the stable period2 motion in the $y$-direction are $a_{20}^{(2)} \approx-0.173500, A_{(2) 1} \approx$ $0.735847, A_{(2) 2} \approx 0.462658, A_{(2) 3} \approx 0.051716$, $A_{(2) 4} \approx 0.034948, A_{(2) 5} \approx 0.020084$, and $A_{(2) 6} \approx$ $5.72 \times 10^{-3}$. However, $A_{(2) 1 / 2} \approx 0.017013, A_{(2) 3 / 2} \approx$ $0.030477, A_{(2) 5 / 2} \approx 0.071263, A_{(2) 7 / 2} \approx 3.06 \times 10^{-3}$, $A_{(2) 9 / 2} \approx 4.11 \times 10^{-3}$, and $A_{(2) 11 / 2} \approx 0.010725$. The other 


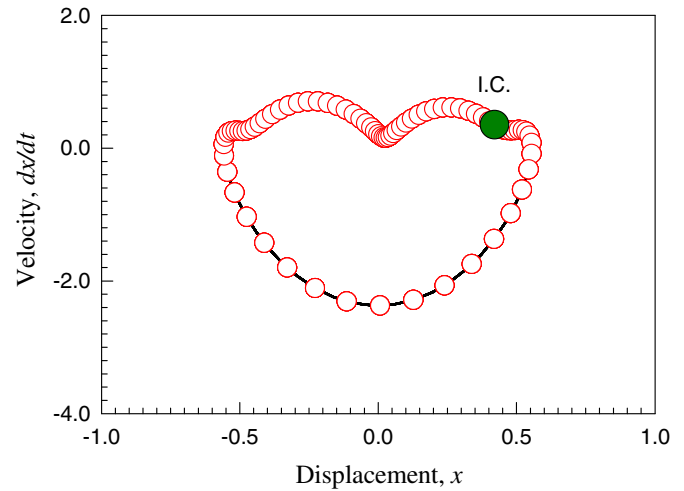

(i)

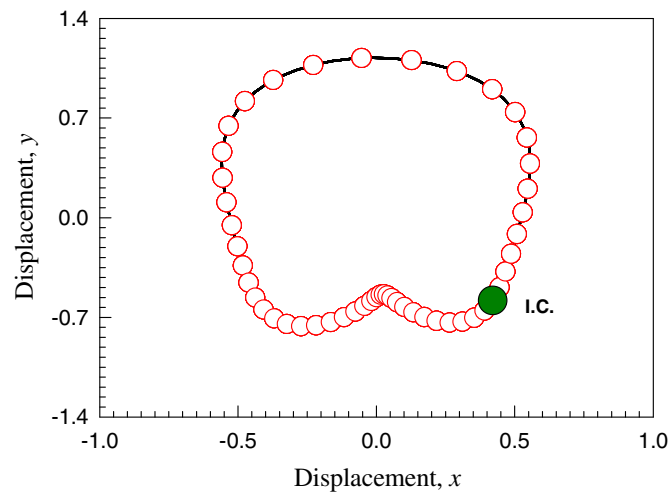

(iii)

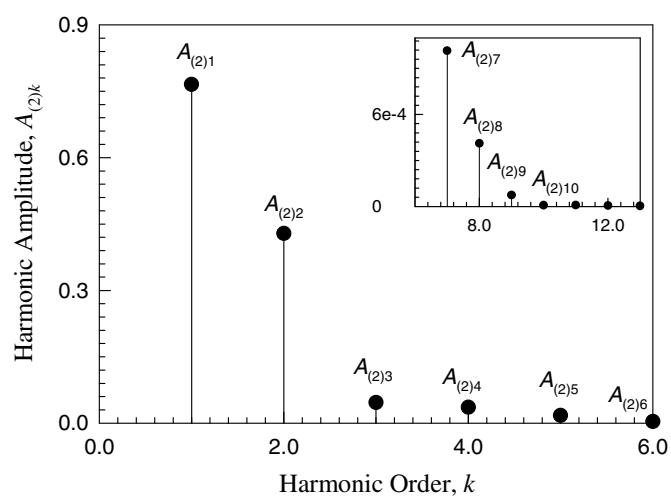

(v)

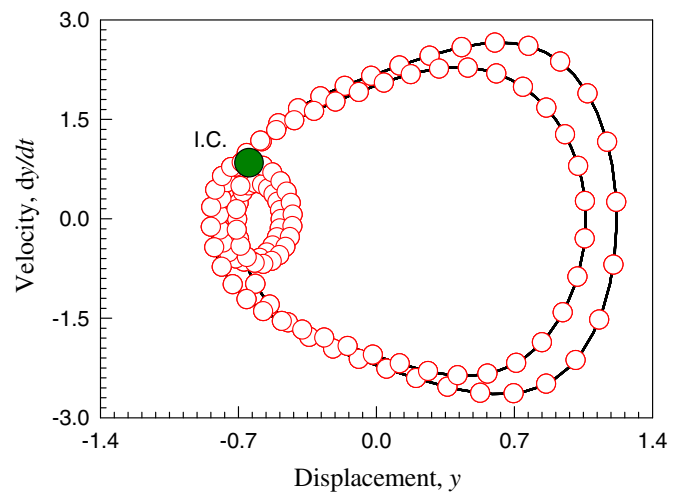

(vii)

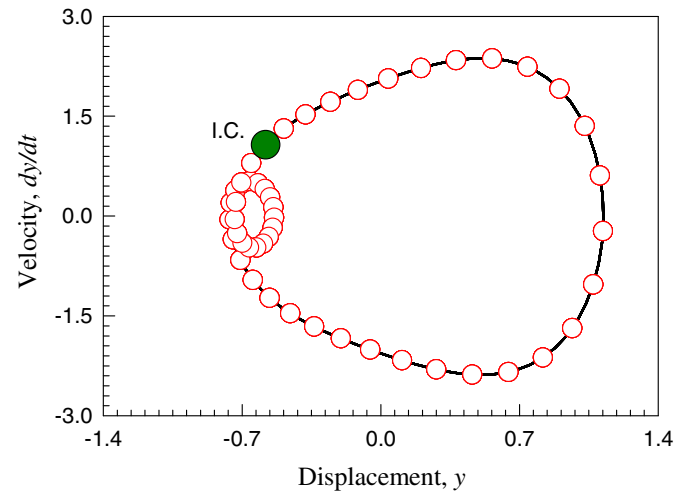

(ii)

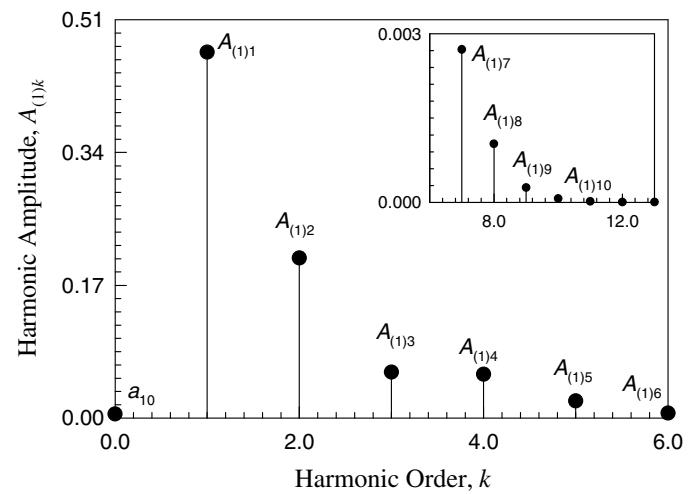

(iv)

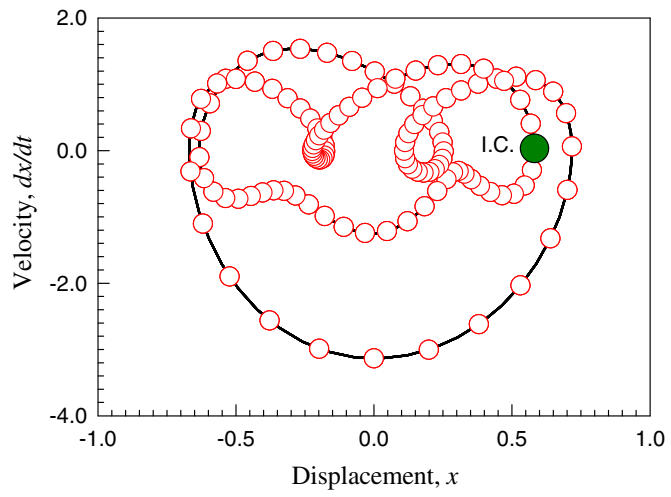

(vi)

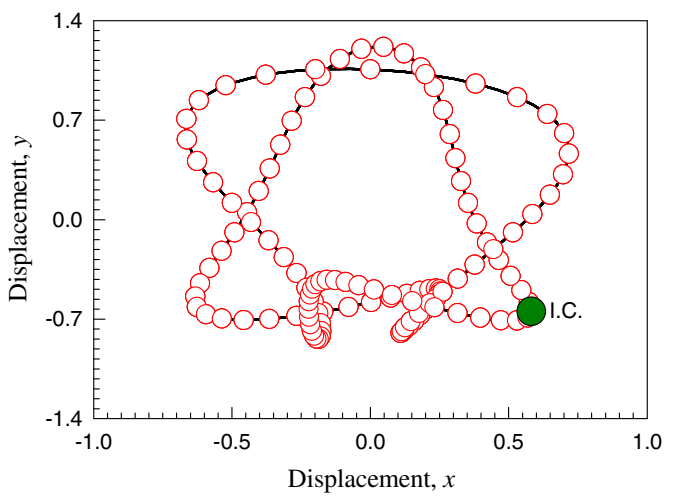

(viii)

Fig. $11 x$-trajectory, $y$-trajectory; displacement orbit, $x$-harmonic amplitude, and $y$-harmonic amplitude for a nonlinear rotor $(\Omega=1.63)$ : (i) $-(\mathrm{v})$ stable period-1 motion (HB13); (vi)-(x) stable period-2 motion (HB26); (xi)-(xv) unstable period-2 motion $(\mathrm{HB} 26)$. $(\delta=0.02, \alpha=0.68, \beta=$ $10, \gamma=1.0, e=1.5$ ) 


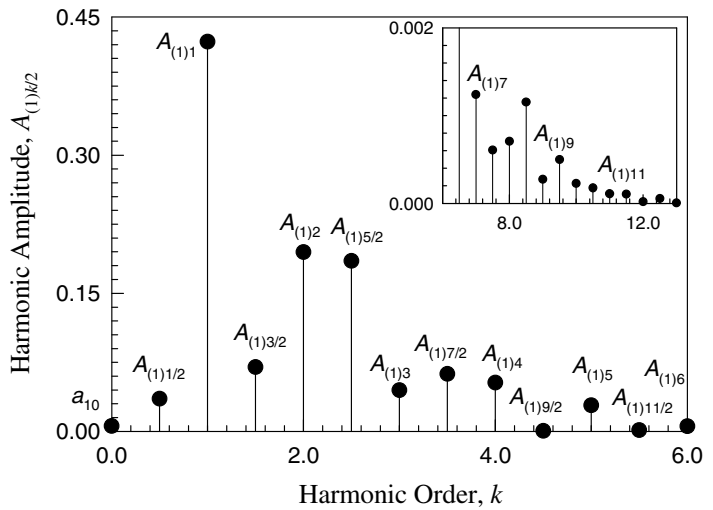

(ix)

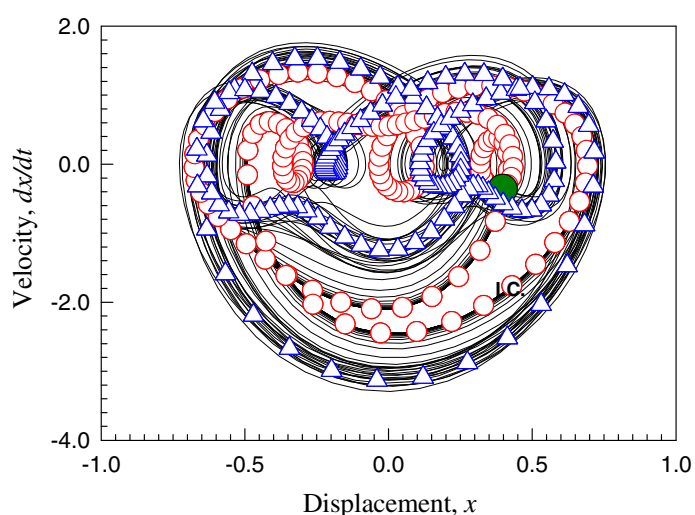

(xi)

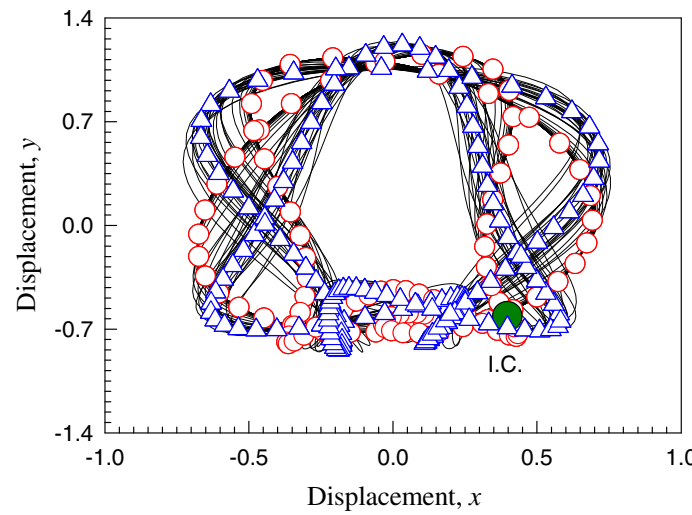

(xiii)

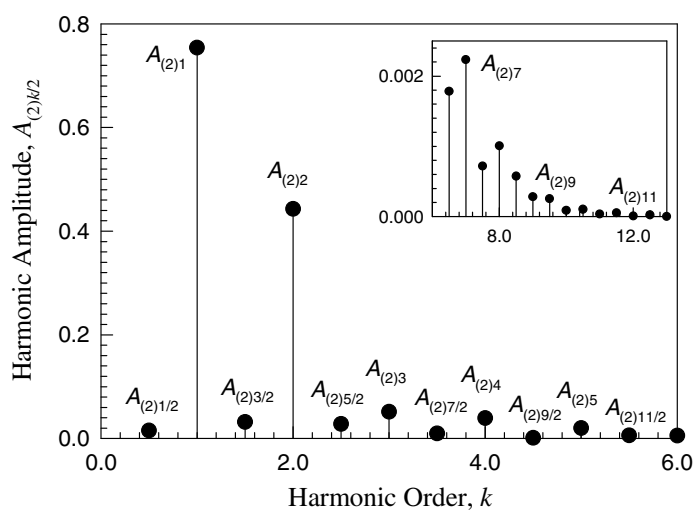

(xv)

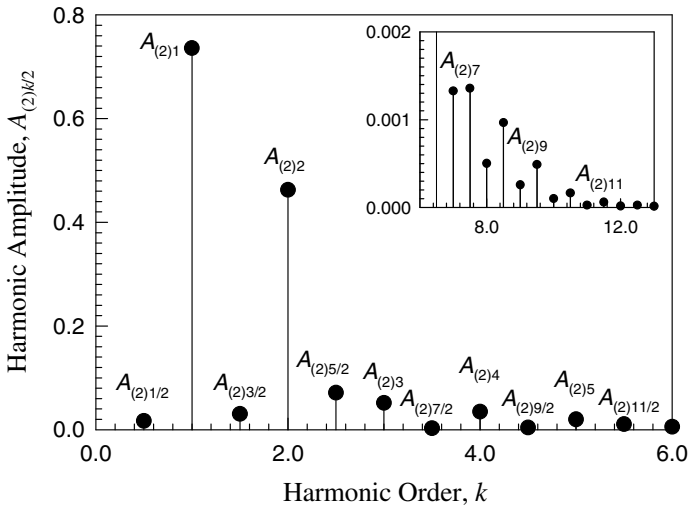

(x)

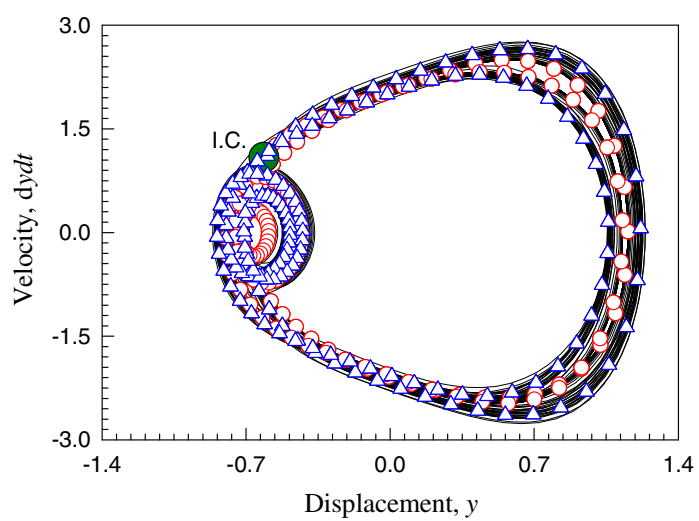

(xii)

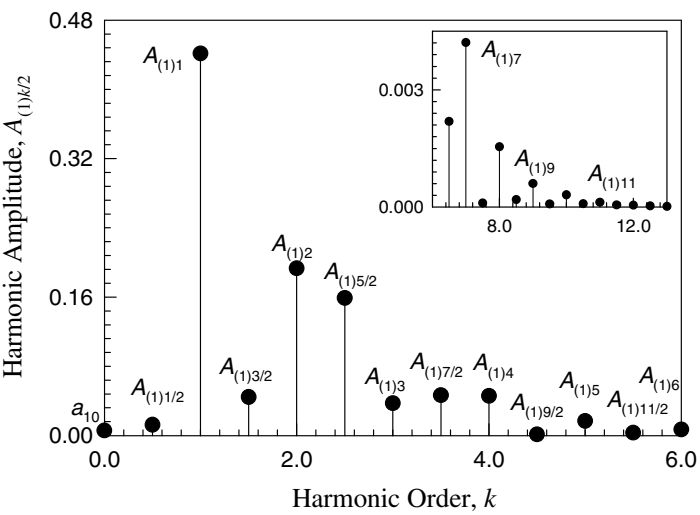

(xiv)

Fig. 11 continued 
Table 1 Input data for numerical simulations $(\Omega=1.63, \quad \delta=0.02, \quad \alpha=0.68, \quad \beta=10, \quad \gamma=1.0, \quad e=1.5)$

\begin{tabular}{|c|c|c|c|c|c|c|}
\hline Figure 11 & $x_{0}$ & $\dot{x}_{0}$ & $y_{0}$ & $\dot{y}_{0}$ & Type & Stability \\
\hline (i) $-(\mathrm{v})$ & 0.420294 & 0.352411 & -0.581414 & 1.070300 & P-1 (HB13) & Stable \\
\hline (vi) $-(\mathrm{x})$ & 0.581743 & 0.030619 & -0.645826 & 0.845058 & P-2 (HB26) & Stable \\
\hline (xi)-(xv) & 0.397202 & 0.362080 & 0.613131 & 1.088330 & P-2 (HB26) & Unstable \\
\hline
\end{tabular}

harmonic amplitudes in the $y$-direction are and $A_{(2) k / 2} \in$ $\left(10^{-7}, 10^{-4}\right)(k=13,14, \ldots 26)$.

The $x$ and $y$-trajectories, displacement orbit and, $x$ and $y$-harmonic spectrum for an unstable period-2 motion are presented in Fig. 11(xi)-(xv) for $\Omega=1.63$, respectively. The analytical solutions based on 26 harmonic terms (HB26). The two trajectories for unstable period-2 motion start from its analytical solution to the close stable period-2 motion presented in Fig. 11(vi)-(viii). For the first few periods, the analytical and numerical results match very well. However, once the computational time is over 10 periods, the numerical solution of the unstable period-2 motion moves away from the analytical solution, and finally such numerical solution arrive to the close stable period- 2 motion, which is very clearly observed. The analytical solution of unstable period- 2 motion is presented by circular symbols, and the stable period-2 motion is presented by the triangular symbols. The $x$ and $y$-harmonic spectrums of the unstable period-2 motion are presented in Fig. 11(xiv), (xv). The main harmonic amplitudes of the unstable period- 2 motions of the nonlinear rotor in the $x$-direction are $a_{10}^{(2)} \approx 5.69 \times$ $10^{-3}, \quad A_{(1) 1} \approx 0.441400 A_{(1) 2} \approx 0.193106, A_{(1) 3} \approx$ $0.037308, A_{(1) 4} \approx 0.045924$, and $A_{(1) 5} \approx 0.016914$. However, $A_{(1) 1 / 2} \approx 0.012372, \quad A_{(1) 3 / 2} \approx 0.044446, A_{(1) 5 / 2} \approx$ $0.159029, A_{(1) 7 / 2} \approx 0.046515$, and $A_{(1) 9 / 2} \approx 1.32 \times$ $10^{-3}$. The other harmonic amplitudes in the $x$-direction are $A_{(1) k / 2} \in\left(10^{-6}, 10^{-3}\right)(k=16,17, \ldots 26)$. However, the main harmonic amplitudes of the unstable period-2 motion in the $y$-direction are $a_{20}^{(2)} \approx-0.173600 A_{(2) 1} \approx$ $0.754332, A_{(2) 2} \approx 0.442879, A_{(2) 3} \approx 0.051271, A_{(2) 4}$ $\approx 0.039417$, and $A_{(2) 5} \approx 0.019897$. However, $A_{(2) 1 / 2} \approx$ $0.014903, A_{(2) 3 / 2} \approx 0.031822, A_{(2) 5 / 2} \approx 0.027851$, $A_{(2) 7 / 2} \approx 9.49 \times 10^{-3}, \quad A_{(2) 9 / 2} \approx 1.05 \times 10^{-3}$, and $A_{(2) 11 / 2} \approx 0.010725$. The other harmonic amplitudes in the $y$-direction are $A_{(2) 6} \approx 5.44 \times 10^{-3}$, and $A_{(2) k / 2} \in$ $\left(10^{-7}, 10^{-4}\right)(k=13,14, \ldots 26)$.

For rotation speed $\Omega=1.947$, a stable period- 1 motion, a stable period- 2 motion and an unstable period- 2 motion also coexist, as shown in Fig. 12. The input data for numerical simulation is listed in Table 2. To reduce illustrations, the time-histories of displacements and velocities in the $x$ and $y$-directions with the $x$ and $y$ - trajectories will not be presented herein. Displacement orbit with $x$ and $y$ harmonic spectrum for period-1 motion are presented in
Fig. 12(i)-(iii) for $\Omega=1.947$. The analytical solutions are still based on 13 harmonic terms (HB13). The displacement orbit is illustrated in Fig. 12(i). The analytical and numerical results match very well for over 40 periods. From the harmonic amplitude spectrum, the main harmonic amplitudes of the stable period- 1 motion in the $x$-direction are $a_{10} \approx$ $0.084514, A_{(1) 1} \approx 0.662876 A_{(1) 2} \approx 0.076260, A_{(1) 3} \approx$ $0.127689, A_{(1) 4} \approx 0.042549$, and $A_{(1) 5} \approx 4.17 \times 10^{-3}$. The other harmonic amplitudes of the stable period-1 motion in the $x$-direction are $A_{(1) k} \in\left(10^{-6}, 10^{-3}\right)(k=$ $6,7, \ldots, 13)$. However, the main harmonic amplitudes of the stable period-1 motion in the $y$-direction are $a_{20} \approx-3.34 \times$ $10^{-3} A_{(2) 1} \approx 0.912159, A_{(2) 2} \approx 0.277731, A_{(2) 3} \approx$ $0.031823, A_{(2) 4} \approx 0.030860$, and $A_{(2) 5} \approx 9.89 \times 10^{-3}$. The other harmonic amplitudes in the $y$-direction are $A_{(2) k} \in$ $\left(10^{-6}, 10^{-3}\right)(k=6,7, \ldots, 13)$.

The displacement orbit with $x$ and $y$-harmonic spectrum for stable period-2 motion are presented in Fig. 12(iv)-(vi) for $\Omega=1.947$. The analytical solutions are based on 26 harmonic terms (HB26). The displacement orbit is shown in Fig. 12(iv). The analytical and numerical results are in a good agreement. The $x$ and $y$ - harmonic spectrums of the stable period- 2 motion are presented in Fig. 12(v), (vi). The main harmonic amplitudes of the stable period-2 motion in the $x$-direction are $a_{10}^{(2)} \approx$ $0.081818, A_{(1) 1} \approx 0.626481, A_{(1) 2} \approx 0.070470, A_{(1) 3} \approx$ $0.119021, A_{(1) 4} \approx 0.051458$, and $A_{(1) 5} \approx 1.59 \times$ $10^{-3}$. However, $A_{(1) 1 / 2} \approx 7.08 \times 10^{-3}, A_{(1) 3 / 2} \approx$ $0.018414, A_{(1) 5 / 2} \approx 0.190927, A_{(1) 7 / 2} \approx 1.46 \times 10^{-3}$, and $A_{(1) 9 / 2} \approx 3.06 \times 10^{-4}$. The other harmonic amplitudes in the $x$-direction are $A_{(1) k / 2} \in\left(10^{-6}, 10^{-3}\right)(k=$ $16,17, \ldots 26)$. However, the main harmonic amplitudes of the stable period-2 motion in the $y$-direction are $a_{20}^{(2)} \approx$ $-3.22 \times 10^{-3}, \quad A_{(2) 1} \approx 0.900758, A_{(2) 2} \approx 0.297508, A_{(2) 3}$ $\approx 0.030919, A_{(2) 4} \approx 0.032236$, and $A_{(2) 5} \approx 0.010609$. However, $A_{(2) 1 / 2} \approx 0.022680, A_{(2) 3 / 2} \approx 0.013130, \quad A_{(2) 5 / 2}$ $\approx 0.034045, \quad A_{(2) 7 / 2} \approx 0.010821, \quad A_{(2) 9 / 2} \approx 0.011712$, and $A_{(2) 11 / 2} \approx 5.58 \times 10^{-3}$. The other harmonic amplitudes in the $y$-direction are $A_{(2) 6} \sim 1.27 \times 10^{-3}$, and $A_{(2) k / 2} \in\left(10^{-7}, 10^{-4}\right)(k=13,14, \ldots 26)$.

The displacement orbit with $x$ and $y$-harmonic spectrum for unstable period-2 motion are presented in Fig. 12(vii)(ix) for $\Omega=1.947$. The analytical solutions are based on 26 harmonic terms (HB26). From displacement orbit in 


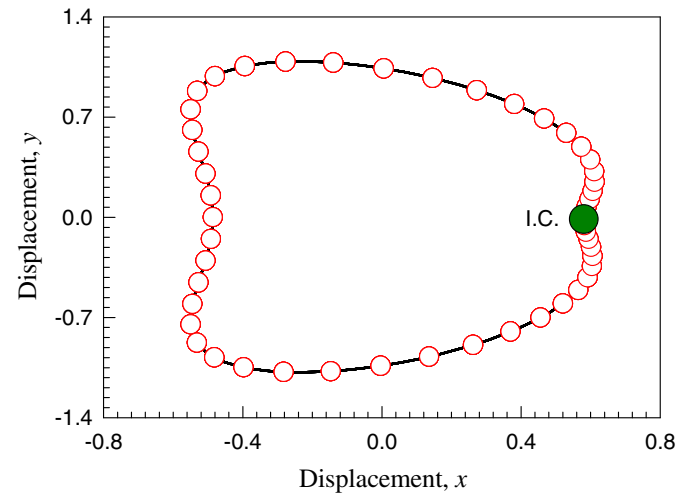

(i)

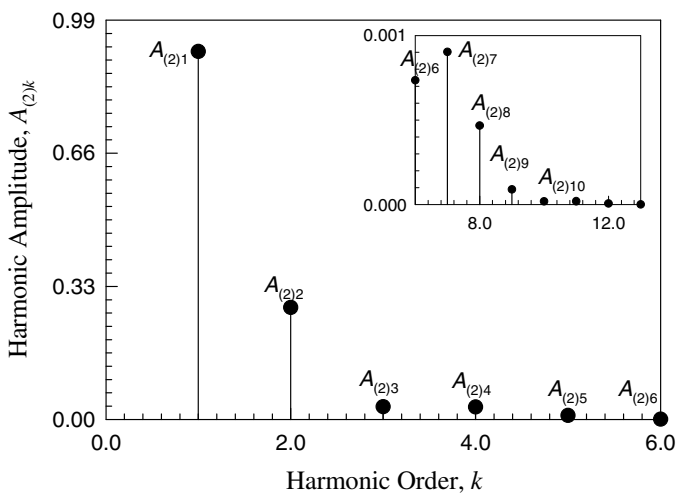

(iii)

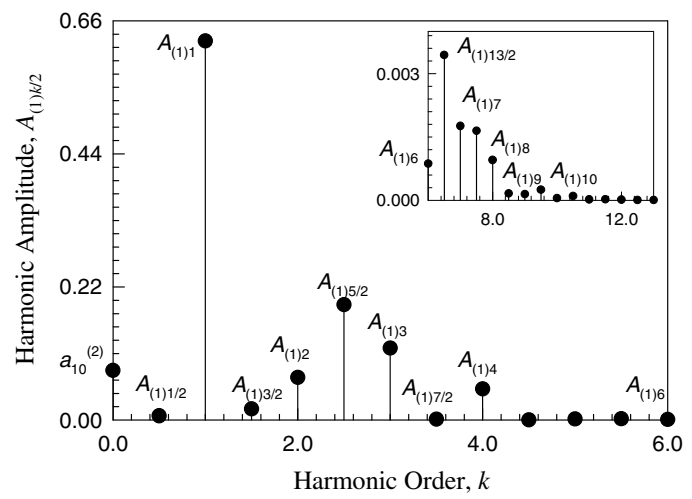

(v)

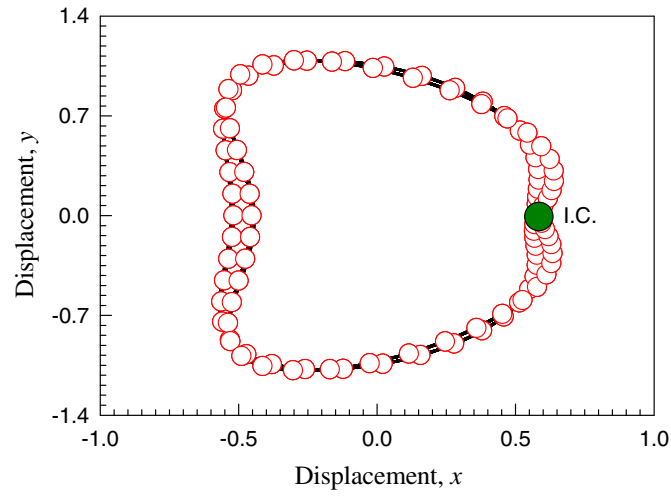

(vii)

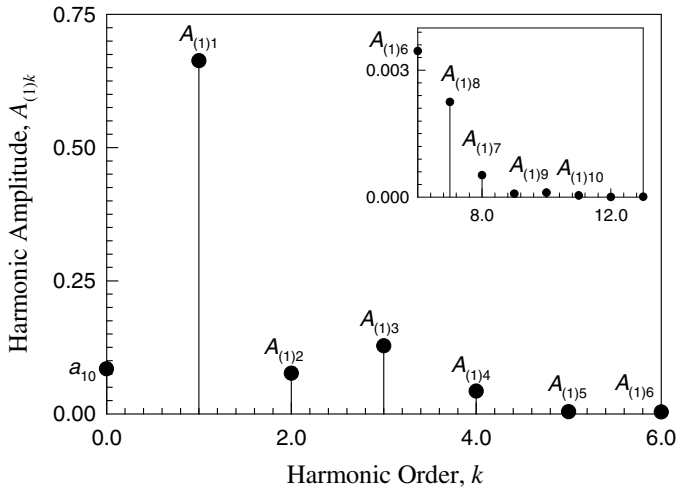

(ii)

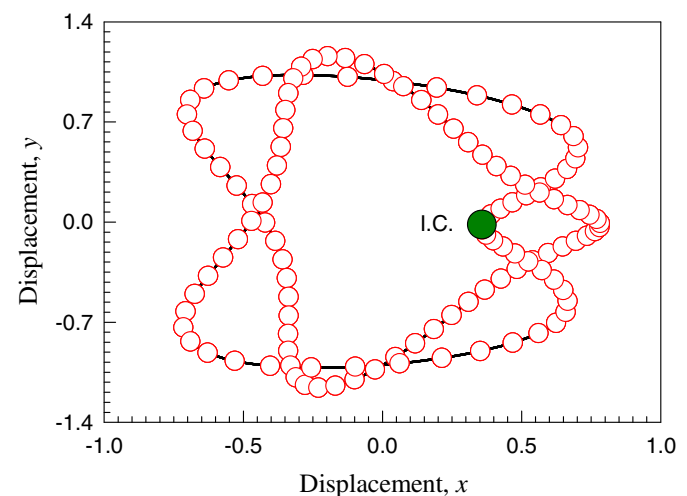

(iv)

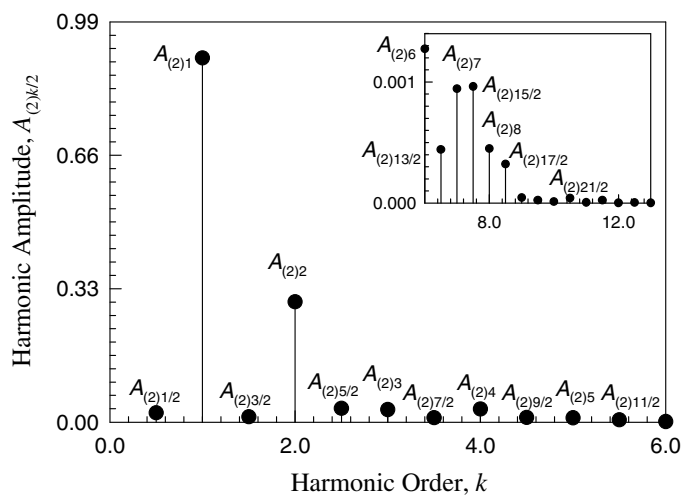

(vi)

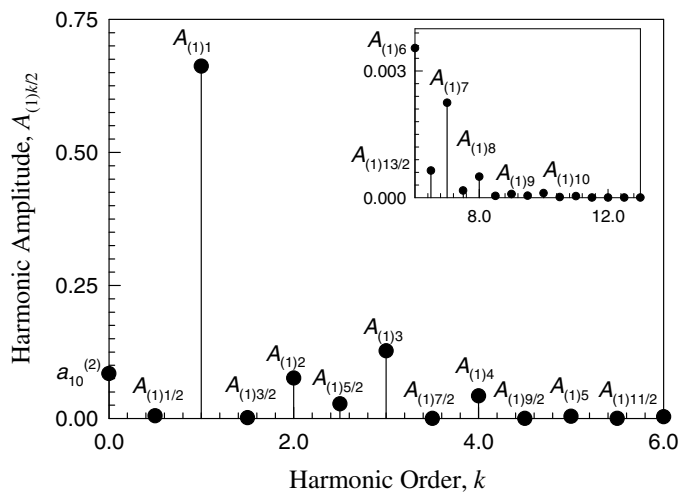

(viii)

Fig. $12 x$-trajectory, $y$-trajectory; displacement orbit, $x$-harmonic amplitude, and $y$-harmonic amplitude for a nonlinear rotor $(\Omega=1.947)$ : (i)-(iii) stable period-1 motion (HB13); (iv)-(vi) stable period-2 motion (HB26); (vii)-(ix) unstable period-2 motion (HB26). $(\delta=0.02, \alpha=0.68, \beta=$ $10, \gamma=1.0, e=1.5)$ 


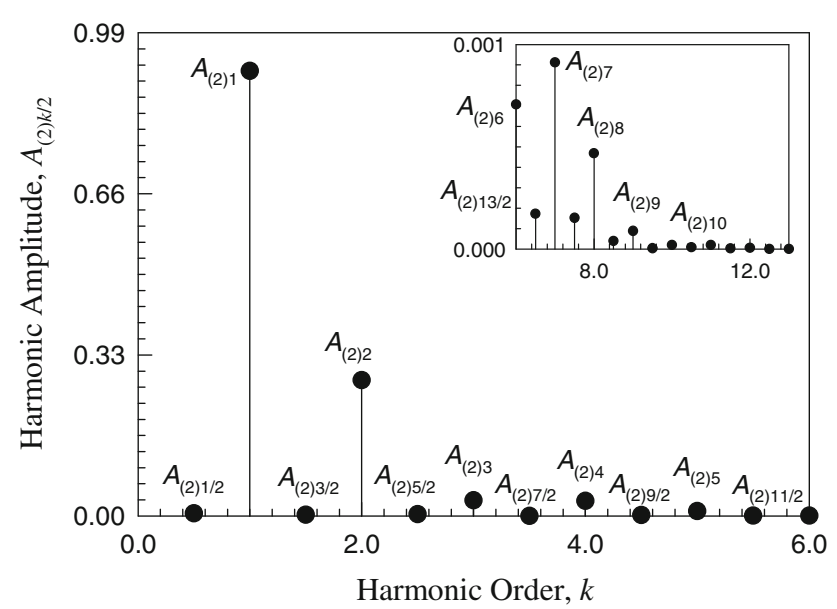

(ix)

Fig. 12 continued

Fig. 12(vii), the analytical and numerical results match very well for over 40 periods. The $x$ and $y$ - harmonic spectrums of the unstable period-2 motion are presented in Fig. 12(viii), (ix). The main harmonic amplitudes of the unstable period-2 motions of the nonlinear rotor in the $x$-direction are $a_{10}^{(2)} \approx$ $0.084603, \quad A_{(1) 1} \approx 0.662095 A_{(1) 2} \approx 0.075991, A_{(1) 3} \approx$ $0.127219, A_{(1) 4} \approx 0.042379$, and $A_{(1) 5} \approx 4.18 \times 10^{-3}$. However, $A_{(1) 1 / 2} \approx 5.21 \times 10^{-3}, A_{(1) 3 / 2} \approx 1.38 \times$ $10^{-3}, A_{(1) 5 / 2} \approx 0.027667, A_{(1) 7 / 2} \approx 1.36 \times 10^{-4}$, and $A_{(1) 9 / 2} \approx 1.14 \times 10^{-4}$. The other harmonic amplitudes in the $x$-direction are $A_{(1) k / 2} \in\left(10^{-6}, 10^{-3}\right)(k=11,12, \ldots 26)$. However, the main harmonic amplitudes of the unstable period-2 motion in the $y$-direction are $a_{20}^{(2)} \approx-3.34 \times$ $10^{-3} A_{(2) 1} \approx 0.911949, A_{(2) 2} \approx 0.278395, A_{(2) 3} \approx$ $0.031864, A_{(2) 4} \approx 0.031001$, and $A_{(2) 5} \approx 9.90 \times 10^{-3}$. However, $A_{(2) 1 / 2} \approx 5.43 \times 10^{-3}, \quad A_{(2) 3 / 2} \approx 2.55 \times$ $10^{-3}, \quad A_{(2) 5 / 2} \approx 3.74 \times 10^{-3}, \quad A_{(2) 7 / 2} \approx 2.62 \times$ $10^{-4}, \quad A_{(2) 9 / 2} \approx 2.05 \times 10^{-3}$, and $A_{(2) 11 / 2} \approx 6.33 \times$ $10^{-4}$. The other harmonic amplitudes in the $y$-direction are $A_{(2) 6} \approx 7.06 \times 10^{-4}$, and $A_{(2) k / 2} \in\left(10^{-7}, 10^{-4}\right)$ $(k=13,14, \ldots 26)$.

For rotation speed $\Omega=1.5871$, one stable period-1 motion, two stable- 2 motions, five unstable period-1 motions and one unstable period- 2 motion coexist. Herein, only a stable period-1 motion, and two stable period-2 motions are shown in Fig. 13. The input data for numerical simulation is listed in Table 3. Displacement orbit with $x$ and $y$-harmonic spectrum for stable period-1 motion are presented in Fig. 13(i)-(iii) for $\Omega=1.5871$. The analytical solutions are still based on 13 harmonic terms (HB13). The displacement orbit is illustrated in Fig. 13(i). In Fig. 13(ii), the main harmonic amplitudes of the stable period- 1 motion in the $x$-direction are $a_{10} \approx 4.59 \times$ $10^{-3}, A_{(1) 1} \approx 0.471394 A_{(1) 2} \approx 0.181789, A_{(1) 3} \approx$ $0.068051, A_{(1) 4} \approx 0.056185$, and $A_{(1) 5} \approx 0.019620$. The other harmonic amplitudes of the stable period-1 motion in the $x$-direction are $A_{(1) k} \in\left(10^{-6}, 10^{-3}\right)(k=$ $6,7, \ldots, 13)$. However, in Fig. 13(iii), the main harmonic amplitudes of the stable period- 1 motion in the $y$-direction are $a_{20} \approx-0.166027, A_{(2) 1} \approx 0.757859, A_{(2) 2} \approx$ $0.391723, A_{(2) 3} \approx 0.046017, A_{(2) 4} \approx 0.035717$, and $A_{(2) 5} \approx 0.015203$. The other harmonic amplitudes in the $y$-direction are $A_{(2) k} \in\left(10^{-6}, 10^{-3}\right)(k=6,7$, ..., 13).

The displacement orbit with $x$ and $y$-harmonic spectrum for the first stable period- 2 motion are presented in Fig. 13(iv)-(vi) for $\Omega=1.5871$. The analytical solutions are based on 26 harmonic terms (HB26). The displacement orbit is shown in Fig. 13(iv). The analytical and numerical results are in a good agreement. The $x$ and $y$-harmonic spectrums of the first stable period-2 motion are presented in Fig. 13(v), (vi). The main harmonic amplitudes of the first stable period-2 motion in the $x$-direction are $a_{10}^{(2)} \approx$ $0.158493, A_{(1) 1} \approx 0.487875, A_{(1) 2} \approx 0.161138, A_{(1) 3} \approx$ $1.12 \times 10^{-3}, \quad A_{(1) 4} \approx 0.043354$, and $A_{(1) 5} \approx 0.035588$. However, $A_{(1) 1 / 2} \approx 3.12 \times 10^{-4}, \quad A_{(1) 3 / 2} \approx 0.018414$, $A_{(1) 5 / 2} \approx 0.011068, \quad A_{(1) 7 / 2} \approx 1.16 \times 10^{-3}$, and $A_{(1) 9 / 2} \sim$ $9.03 \times 10^{-5}$. The other harmonic amplitudes in the $x$ direction are $A_{(1) k / 2} \in\left(10^{-6}, 10^{-3}\right)(k=16,17, \ldots 26)$. However, the main harmonic amplitudes of the stable period2 motion in the $y$-direction are $a_{20}^{(2)} \approx-0.030528, \quad A_{(2) 1} \approx$ $0.489041, A_{(2) 2} \approx 0.651005, A_{(2) 3} \approx 0.126329, A_{(2) 4}$ $\approx 9.47 \times 10^{-3}$, and $A_{(2) 5} \approx 0.031786$. However, $A_{(2) 1 / 2} \approx$ $3.25 \times 10^{-3}, \quad A_{(2) 3 / 2} \approx 1.19 \times 10^{-3}, \quad A_{(2) 5 / 2} \approx 1.0 \times$ $10^{-3}, \quad A_{(2) 7 / 2} \approx 8.32 \times 10^{-4}, \quad A_{(2) 9 / 2} \approx 5.16 \times 10^{-4}$, and $A_{(2) 11 / 2} \approx 3.23 \times 10^{-4}$. The other harmonic amplitudes in the $y$-direction are $A_{(2) 6} \approx 0.020208$, and $A_{(2) k / 2} \in$ $\left(10^{-7}, 10^{-3}\right)(k=13,14, \ldots 26)$. From the harmonic amplitudes, the harmonic amplitudes $A_{(i)(2 l-1) / 2}$ is very small compared to $A_{(i)(2 l) / 2}(l=1,2, \ldots)$. So the period- 2 motion is very close to period-1 motion.

Table 2 Input data for numerical simulations $(\Omega=1.947, \delta=0.02, \alpha=0.68, \beta=10, \gamma=1.0, e=1.5)$

\begin{tabular}{lllllll}
\hline Figure 12 & $x_{0}$ & $\dot{x}_{0}$ & $y_{0}$ & $\dot{y}_{0}$ & Type & Stability \\
\hline (i)-(v) & 0.580111 & $8.928900 \mathrm{E}-3$ & -0.013079 & 0.668064 & P-1 (HB13) & Stable \\
(vi)-(x) & 0.357099 & 0.085054 & -0.017600 & 0.644516 & P-2 (HB26) & Stable \\
(xi)-(xv) & 0.584629 & -0.123069 & $-7.369860 \mathrm{E}-3$ & 0.665021 & P-2 (HB26) & Unstable
\end{tabular}



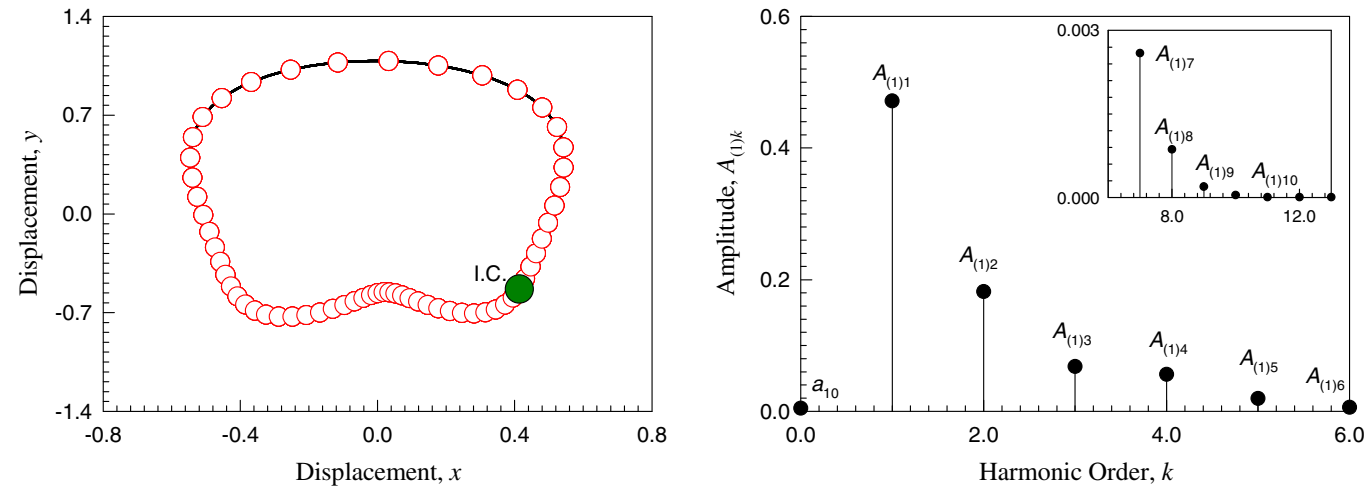

(i)

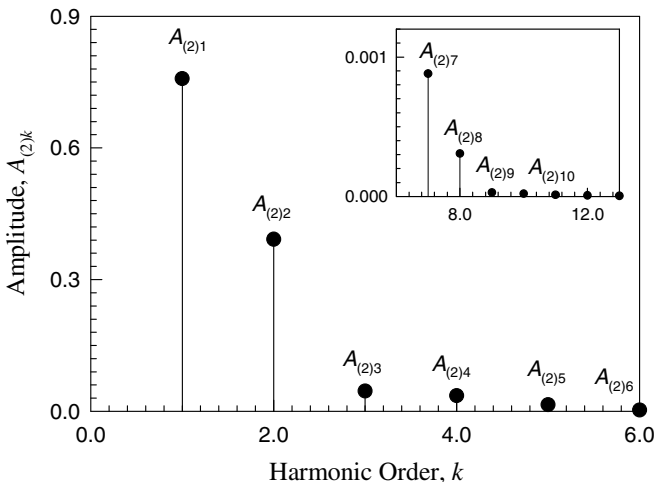

(iii)

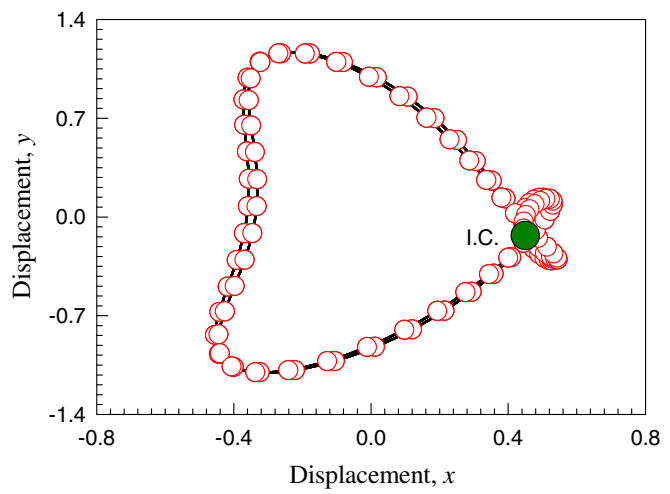

(iv)
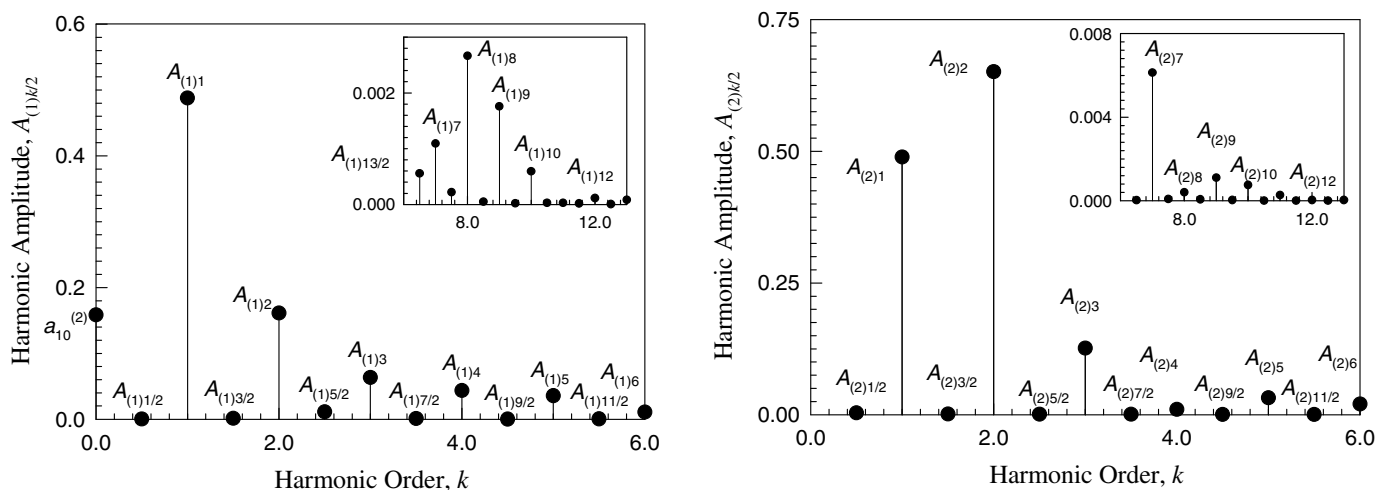

(v)

(vi)

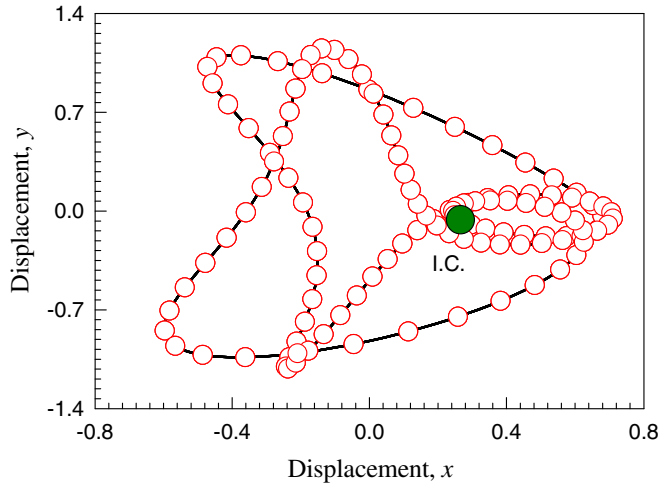

(vii)

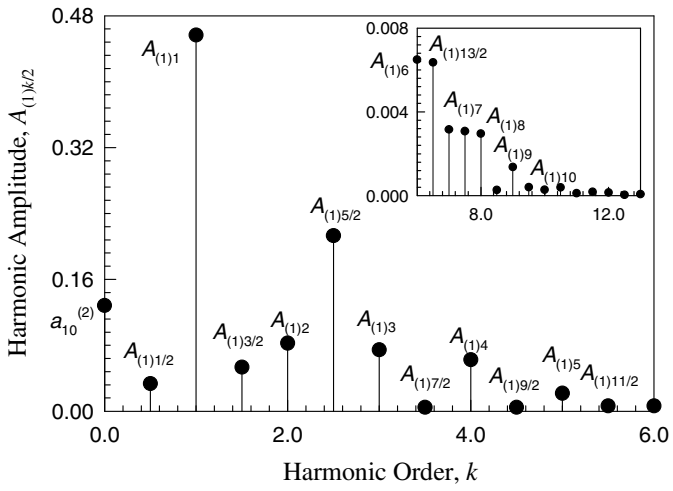

(viii)

Fig. 13 Displacement orbit, $x$-harmonic amplitude, and $y$-harmonic amplitude for a nonlinear rotor $(\Omega=1.5871)$ : (i)-(iii) stable period-1 motion (HB13); (iv)-(vi) the first stable period-2 motion (HB26); (vii)-(ix) the second stable period-2 motion (HB26). $(\delta=0.02, \alpha=0.68, \beta=10, \gamma=$ $1.0, e=1.5)$ 


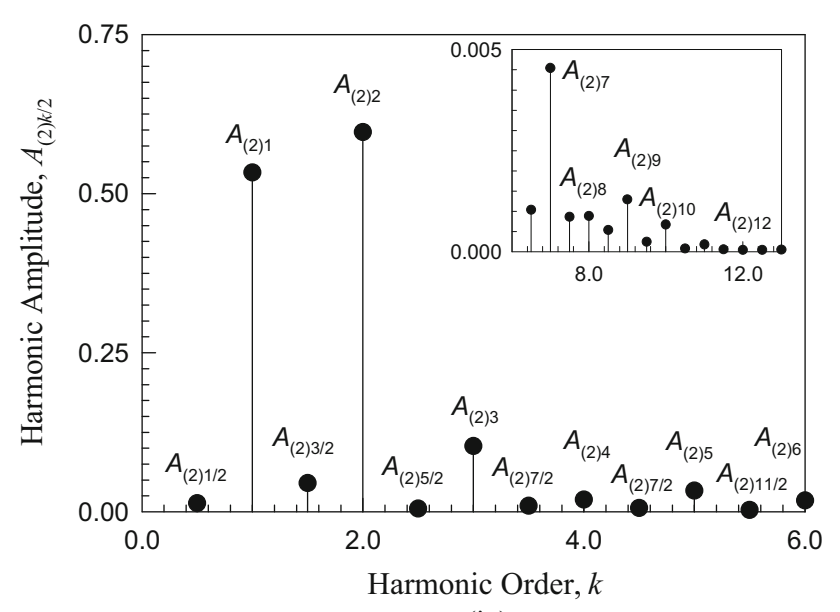

(ix)

Fig. 13 continued

The displacement orbit with $x$ and $y$-harmonic spectrum for the second stable period-2 motion are presented in Fig. 13(vii)-(ix) for $\Omega=1.5871$. The analytical solutions are based on 26 harmonic terms (HB26). The displacement orbit of the second stable period-2 motion is presented in Fig. 13(vii), and the analytical and numerical results match very well for over 40 periods. The $x$ and $y$ - harmonic spectrums of the second stable period-2 motion are presented in Fig. 13(viii), (ix). The main harmonic amplitudes of the second stable period-2 motions of the nonlinear rotor in the $x$-direction are $a_{10}^{(2)} \approx 0.128222, A_{(1) 1} \approx$ $0.456457 A_{(1) 2} \approx 0.082550, A_{(1) 3} \approx 0.074457, A_{(1) 4} \approx$ 0.062591 , and $A_{(1) 5} \approx 0.021721$. However, $A_{(1) 1 / 2} \approx$ $0.033482, A_{(1) 3 / 2} \approx 0.053424, A_{(1) 5 / 2} \approx 0.213030, A_{(1) 7 / 2}$ $\approx 4.67 \times 10^{-3}$, and $A_{(1) 9 / 2} \approx 4.71 \times 10^{-3}$. The other harmonic amplitudes in the $x$-direction are $A_{(1) k / 2} \in$ $\left(10^{-6}, 10^{-3}\right)(k=11,12, \ldots 26)$. However, the main harmonic amplitudes of the second stable period- 2 motion in the $y$-direction are $a_{20}^{(2)} \approx-0.019451, A_{(2) 1} \approx 0.533340, A_{(2) 2}$ $\approx 0.596780, A_{(2) 3} \approx 0.103204, A_{(2) 4} \approx 0.019243$, and $A_{(2) 5} \approx 0.033128$. However, $A_{(2) 1 / 2} \approx 0.013636, A_{(2) 3 / 2} \approx$ $0.045071, A_{(2) 5 / 2} \approx 5.04 \times 10^{-3}, A_{(2) 7 / 2} \approx 9.40 \times$ $10^{-3}, A_{(2) 9 / 2} \approx 6.03 \times 10^{-3}$, and $A_{(2) 11 / 2} \approx 2.97 \times 10^{-3}$. The other harmonic amplitudes in the $y$-direction are $A_{(2) 6} \approx$ 0.017679 , and $A_{(2) k / 2} \in\left(10^{-7}, 10^{-3}\right)(k=13,14, \ldots 26)$.

From the bifurcation analysis, after some Hopf bifurcations, the period- 1 and period- 2 motions become quasi- periodic motions. From the unstable period- 1 motions, the quasi-periodic motions are presented in Fig. 14. The input data for numerical simulation is listed in Table 4. The analytical solutions of unstable period- 1 motions are based on 13 harmonic terms (HB13). The quasi-periodic motions are presented after 1000 periods (1000T) from the analytical unstable period-1 motion. In Fig. 14 (i)-(iii), displacement orbit with $x$ and $y$-harmonic spectrum for quasi-periodic motion relative to unstable period- 1 motion are presented for $\Omega=0.573$. The displacement orbit of the quasi-periodic motion is illustrated in Fig. 14(i). The unstable period-1 motion is the central curves of the quasi-periodic motion, which is depicted by the circular symbol. In Fig. 14(ii), the main harmonic amplitudes of the unstable period-1 motion in the $x$-direction are $A_{(1) 1} \approx 0.225903, A_{(1) 3} \approx 0.181789$, and $A_{(1) 5} \approx 0.056185$. The other harmonic amplitudes of the unstable period- 1 motion in the $x$-direction are $A_{(1) 2 l+1} \in$ $\left(10^{-6}, 10^{-3}\right)(l=3,4, \ldots, 6)$. Since the unstable period1 motion is symmetric, $a_{10} \approx 0$, and $A_{(1) 2 l}=0(l=$ $1,2, \ldots)$ are obtained. However, in Fig. 14(iii), the main harmonic amplitudes of the stable period- 1 motion in the $y$-direction are $A_{(2) 1} \approx 0.306048, A_{(2) 3} \approx 0.114292$ and $A_{(2) 5} \approx 0.019007$. The other harmonic amplitudes in the $y$-direction are $A_{(2) 2 l+1} \in\left(10^{-6}, 10^{-3}\right)(l=3,4, \ldots, 6)$. Since the unstable period- 1 motion is symmetric, $a_{20} \approx 0$, and $A_{(2) 2 l}=0(l=1,2, \ldots)$ are obtained.

The displacement orbit with $x$ and $y$-harmonic spectrum for quasi-periodic motion relative to the unstable period-1 motion are presented in Fig. 14(iv)-(vi) for $\Omega=0.6$. The displacement orbit of the quasi-periodic motion is shown in Fig. 14(iv). The analytical solution of the unstable period-1 motion is also presented by circular symbols, which is the central curve of the quasi-periodic motion. Such a quasiperiodic motion experiences a large amplitude, compared to the previous quasi-periodic motion for $\Omega=0.573$. In Fig. 14(v), the main harmonic amplitudes of the unstable period1 motion in the $x$-direction are $A_{(1) 1} \approx 0.235004 A_{(1) 3} \approx$ 0.049452 , and $A_{(1) 5} \approx 0.016000$. The other harmonic amplitudes of the unstable period- 1 motion in the $x$-direction are $A_{(1) 2 l+1} \in\left(10^{-5}, 10^{-4}\right)(l=3,4, \ldots, 6)$. Since the unstable period- 1 motion is also symmetric, $a_{10} \approx 0$, and $A_{(1) 2 l}=0(l=1,2, \ldots)$ are obtained. In Fig. 14(vi), the main harmonic amplitudes of the stable period-1 motion in the $y$-direction are $A_{(2) 1} \approx 0.324243, A_{(2) 3} \approx 0.112356$ and

Table 3 Input data for numerical simulations $(\Omega=1.5871, \quad \delta=0.02, \alpha=0.68, \quad \beta=10, \quad \gamma=1.0, \quad e=1.5)$

\begin{tabular}{|c|c|c|c|c|c|c|}
\hline Figure 13 & $x_{0}$ & $\dot{x}_{0}$ & $y_{0}$ & $\dot{y}_{0}$ & Type & Stability \\
\hline (i)-(iii) & 0.413698 & 0.270643 & -0.533378 & 1.028980 & P-1 (HB13) & Stable \\
\hline (iv)-(vi) & 0.449954 & 0.121495 & -0.131746 & -0.753568 & P-2 (HB26) & Stable \\
\hline (vii)-(vii) & 0.265762 & 0.366576 & -0.062736 & -0.502230 & P-2 (HB26) & Stable \\
\hline
\end{tabular}




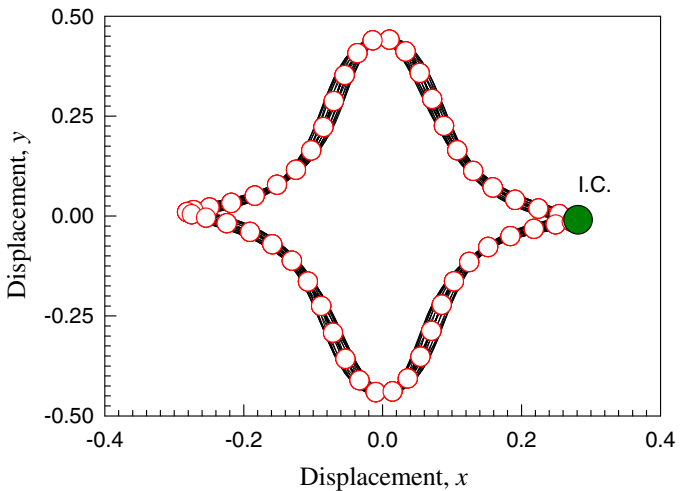

(i)

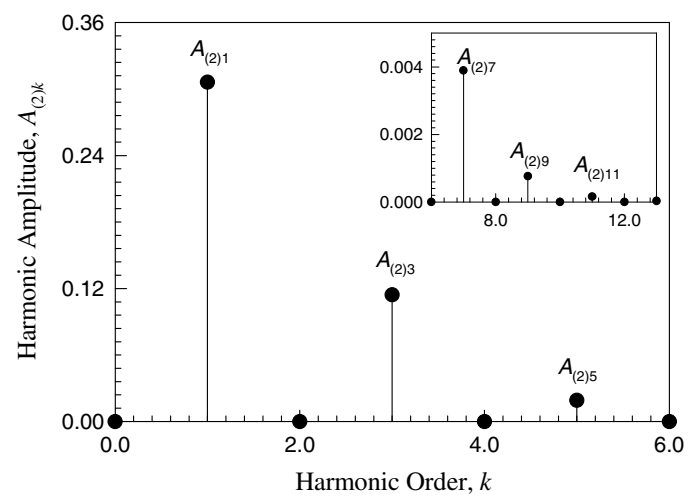

(iii)

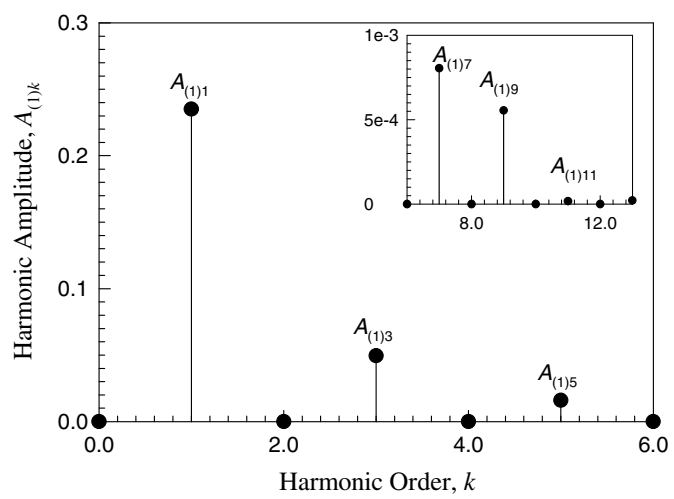

(v)

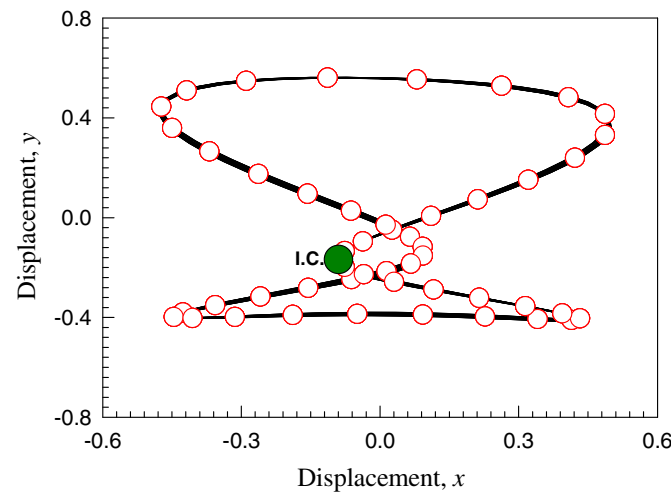

(vii)

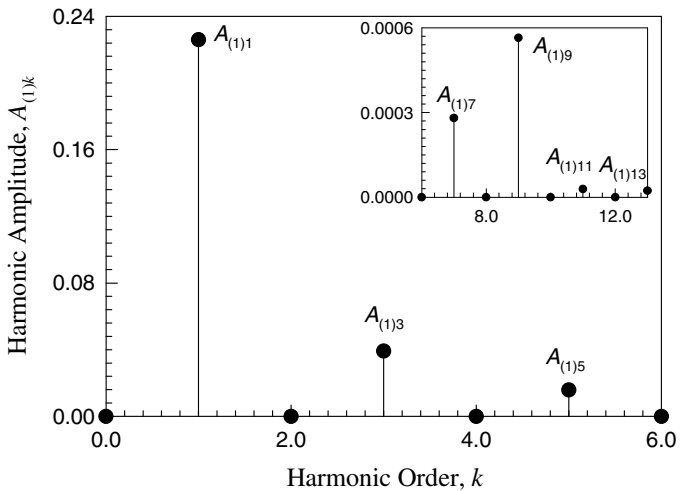

(ii)

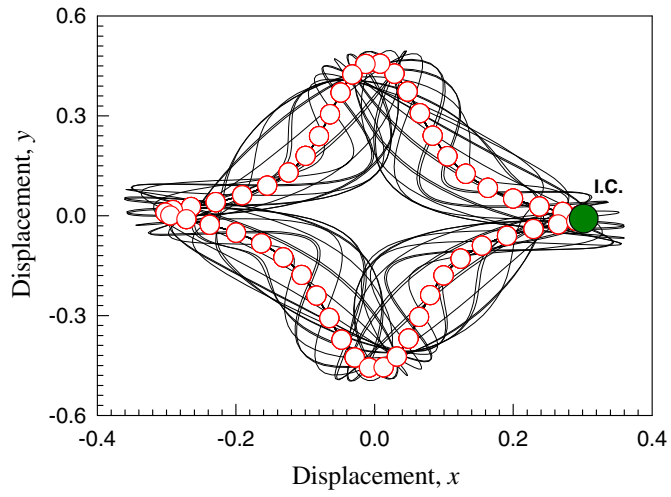

(iv)

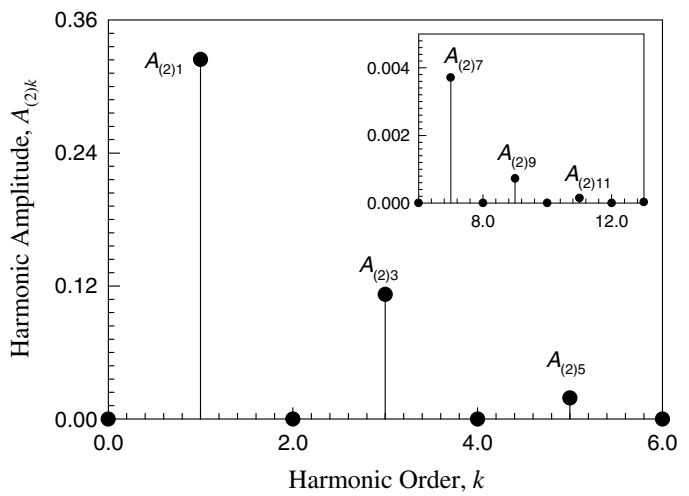

(vi)

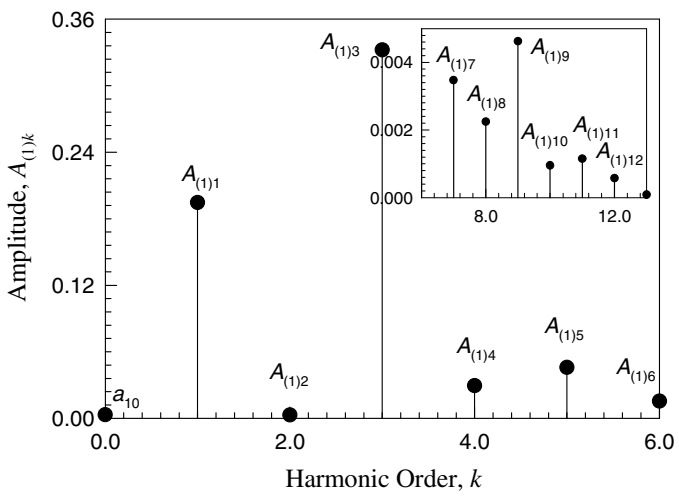

(viii)

Fig. 14 Displacement orbit, $x$-harmonic amplitude, and $y$-harmonic amplitude of quasi-periodic motions relative to period- 1 motion for a nonlinear rotor: (i)-(iii) $\Omega=0.573$ (HB13); (iv)-(vi) $\Omega=0.6$ (HB13); (vii)-(ix) $\Omega=0.896$ (HB13). $(\delta=0.02, \alpha=0.68, \beta=10, \gamma=1.0, e=1.5$ ) 


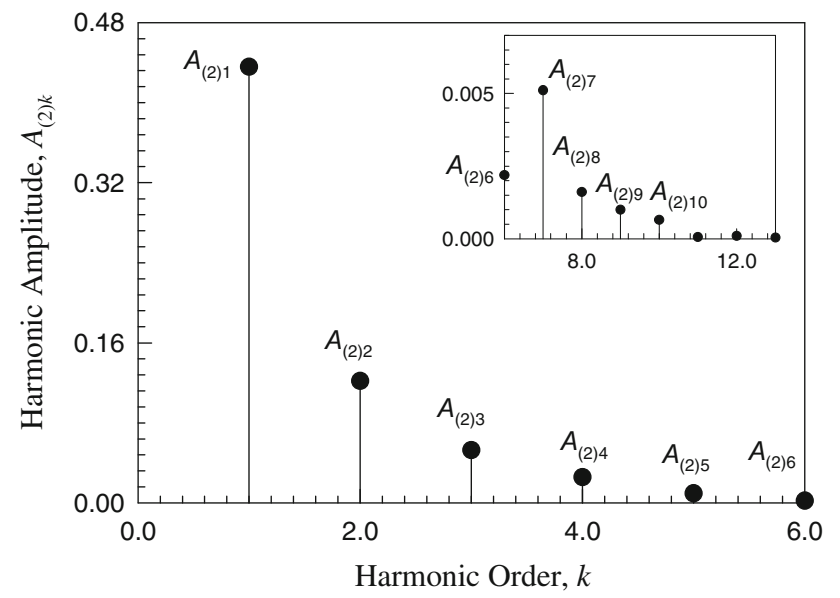

(ix)

Fig. 14 continued

$A_{(2) 5} \approx 0.018902$. The other harmonic amplitudes in the $y$-direction are $A_{(2) 2 l+1} \in\left(10^{-5}, 10^{-3}\right)(l=3,4, \ldots, 6)$. Since the unstable period- 1 motion is symmetric, $a_{20} \approx 0$, and $A_{(2) 2 l}=0(l=1,2, \ldots)$ are obtained.

The displacement orbit with $x$ and $y$-harmonic spectrum for a quasi-periodic motion relative to the unstable, asymmetric period-1 motion are presented in Fig. 14(vii)-(ix) for $\Omega=0.896$. The displacement orbit of the quasi-periodic motion is presented in Fig. 14(vii), and the unstable asymmetric period-1 motion is also presented via the circular symbols, which is still the central curve of the quasi-periodic motion. The $x$ and $y$-harmonic spectrums of the unstable period-1 motion are presented in Fig. 14(viii), (ix). The main harmonic amplitudes of the second stable period- 1 motions of the nonlinear rotor in the $x$-direction are $a_{10} \approx 3.24 \times 10^{-3}, A_{(1) 1} \approx$ $0.194586, A_{(1) 2} \approx 3.00 \times 10^{-3}, A_{(1) 3} \approx 0.332163, A_{(1) 4} \approx$ $0.029424, A_{(1) 5} \approx 0.045866$, and $A_{(1) 6} \approx 0.015542$. The other harmonic amplitudes in the $x$-direction are $A_{(1) k} \in$ $\left(10^{-6}, 10^{-3}\right)(k=7,8, \ldots, 13)$. However, the main harmonic amplitudes of the unstable period- 1 motion in the $y$ direction are $a_{20} \approx-0.060800, A_{(2) 1} \approx 0.435707, A_{(2) 2} \approx$ $0.121917, A_{(2) 3} \approx 0.052810, A_{(2) 4} \approx 0.025832, A_{(2) 5} \approx$ $9.56 \times 10^{-3}$, and $A_{(2) 6} \approx 2.81 \times 10^{-3}$. The other harmonic amplitudes in the $y$-direction are $A_{(2) k} \in\left(10^{-5}, 10^{-3}\right)$ $(k=7,8, \ldots, 13)$.
For the Hopf bifurcation of symmetric period-1 motion at $\Omega=2.859$, the period- 1 motion jumps to the period-5 motion. The symmetric period- 5 motion is observed. In Fig. 15, a period-5 motion based on 55 harmonic terms (HB55) are presented for $\Omega=2.7$. The time-histories of displacement and velocity in the $x$-direction of the nonlinear rotor are presented in Fig. 15(i), (ii), respectively. Two periods (5T) for the period-5 motion is labeled in the two plots. The time-histories of displacement and velocity in the $y$ direction of the nonlinear rotor are also presented in Fig. 15(iii), (iv), respectively. The two trajectories for $x$ and $y$ directions are presented for over 40 periods in Fig. 15 (v), (vi), respectively. The initial conditions are marked by large circular symbols and also labeled by "I.C.". The displacement orbit of the nonlinear rotor in the $x$ and $y$-directions is presented in Fig. 15(vii). To show harmonic contributions on the period-5 motion, the harmonic amplitude spectrums of the stable period-5 motion of the nonlinear rotor in $x$ and $y$-directions are presented in Fig. 15(viii), (ix). The harmonic amplitude spectrums of the stable period-5 motion are given by analytical solutions. The main harmonic amplitudes in the $x$-direction are $A_{(1) 1 / 5} \approx 0.031127, A_{(1) 3 / 5} \approx$ $0.033035, A_{(1) 1} \approx 0.614421, A_{(1) 7 / 5} \approx 0.083210, A_{(1) 9 / 5} \approx$ $0.092028, A_{(1) 11 / 5} \approx 0.550803, A_{(1) 13 / 5} \approx 0.029438, A_{(1) 3} \approx$ $0.025041, A_{(1) 17 / 5} \approx 0.066635, A_{(1) 19 / 5} \approx 0.047846$, $A_{(1) 21 / 5} \approx 0.012867, A_{(1) 23 / 5} \approx 2.76 \times 10^{-3}, A_{(1) 5} \approx$ 0.014469 , and $A_{(1) 27 / 5} \approx 0.014365$. The other harmonic amplitudes in the $x$-direction are $A_{(1)(2 l+1) / 5} \in\left(10^{-5}, 10^{-3}\right)$ $(l=14,15, \ldots 26)$. Due to symmetric period-5 motion, $a_{20}^{(5)} \approx 0$ and $A_{(2) 2 l / 5}=0(l=1,2, \ldots)$. The main harmonic amplitudes in the $y$-direction are $A_{(2) 1 / 5} \approx$ $0.105302, A_{(2) 3 / 5} \approx 0.059754, A_{(2) 1} \approx 1.152670, A_{(2) 7 / 5} \approx$ $0.073500, A_{(2) 9 / 5} \approx 0.416582, A_{(2) 11 / 5} \approx 0.045259, A_{(2) 13 / 5} \approx$ $0.050286, A_{(2) 3} \approx 0.044222, A_{(2) 17 / 5} \approx 0.045093, A_{(2) 19 / 5} \approx$ $0.043544, A_{(2) 21 / 5} \approx 6.83 \times 10^{-3}, A_{(2) 23 / 5} \approx 0.012223$, $A_{(2) 5} \approx 5.42 \times 10^{-3}$, and $A_{(2) 27 / 5} \approx 0.013007$. The other harmonic amplitudes in the $y$ direction are $A_{(2)(2 l+1) / 5} \in$ $\left(10^{-5}, 10^{-3}\right)(l=14,15, \ldots 26)$.

\section{Conclusions}

In this paper, analytical solutions of periodic solutions were developed, and the corresponding stability and bifurcation

Table 4 Input data for numerical simulations of quasi-periodic motions $(\delta=0.02, \quad \alpha=0.68, \quad \beta=10, \quad \gamma=1.0, e=1.5)$

\begin{tabular}{lllllll}
\hline Figure 14 & \multicolumn{1}{l}{$x_{0}$} & $\dot{x}_{0}$ & $y_{0}$ & $\dot{y}_{0}$ & Type & Stability \\
\hline (i)-(iii) $(\Omega=0.573)$ & 0.281391 & $6.698350 \mathrm{e}-3$ & $-9.653220 \mathrm{e}-3$ & 0.021055 & P-1 (HB13) & Unstable \\
(iv)-(vi) $(\Omega=0.6)$ & 0.301636 & $9.452720 \mathrm{e}-3$ & $-8.685960 \mathrm{e}-3$ & 0.036671 & P-1 (HB13) & Unstable \\
(vii)-(ix) $(\Omega=0.896)$ & -0.089768 & $4.633820 \mathrm{e}-4$ & -0.167986 & 0.226290 & P-1 (HB13) & Unstable \\
\hline
\end{tabular}




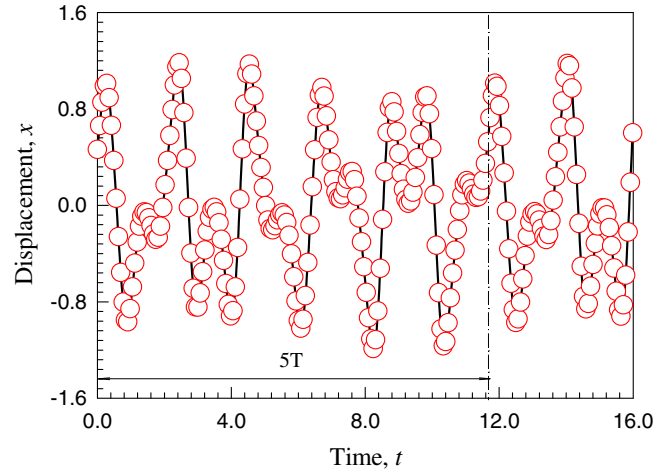

(i)

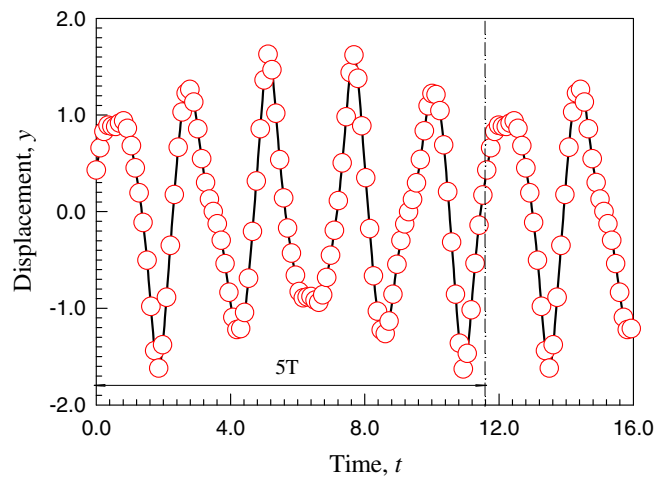

(iii)

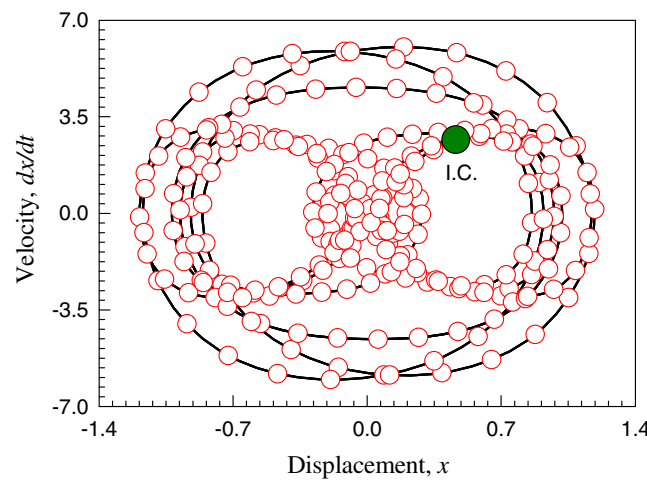

(v)

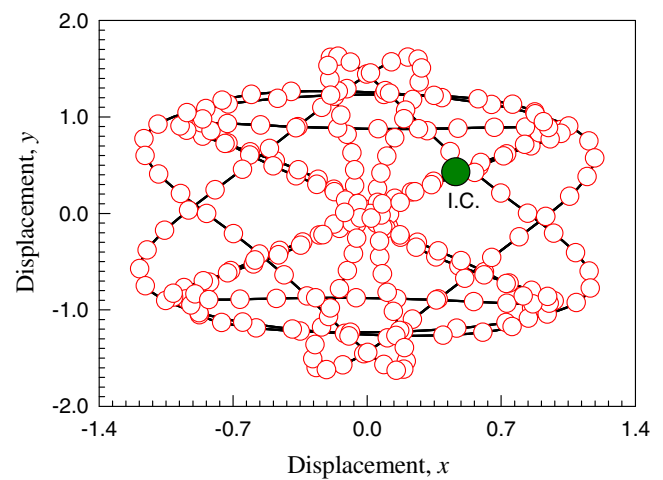

(vii)

Fig. 15 Independent period-5 motion for a nonlinear rotor $(\Omega=$ 2.7, HB55): (i) $x$-displacement, (ii) $x$-velocity; (iii) $y$-displacement, (iv) $y$-velocity; (v) $x$-trajectory and (vi) $y$-trajectory; (vii) displacement orbit, (viiio $x$-harmonic amplitude, and (ix) $y$-harmonic ampli-

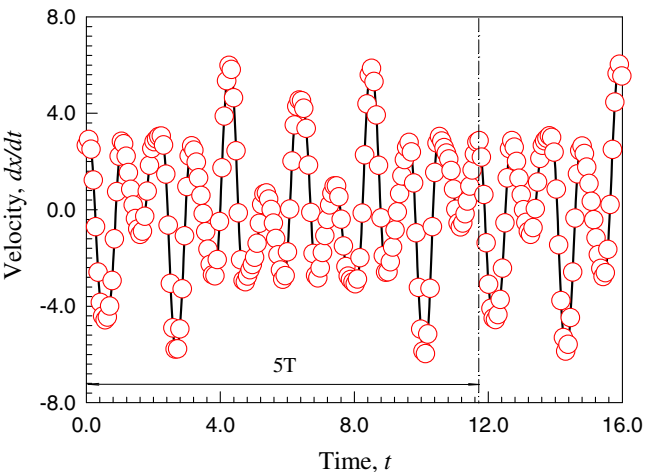

(ii)

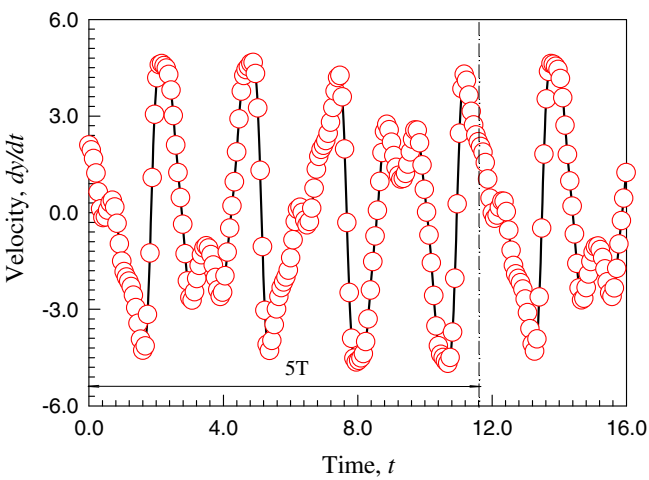

(iv)

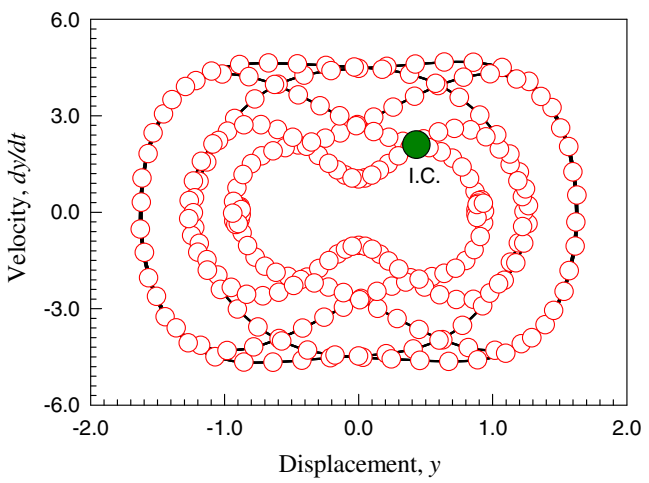

(vi)

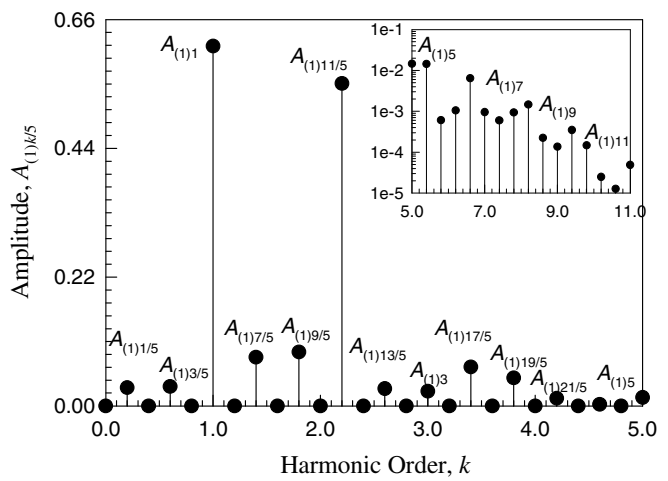

(viii)

tude. Initial condition $\left(x_{0}, \dot{x}_{0}\right)=(0.463286,2.668610)$ and $\left(y_{0}, \dot{y}_{0}\right)=$ (0.430493,2.100350). $(\delta=0.02, \alpha=0.68, \beta=10, \gamma=1.0, e=$ $1.5)$ 


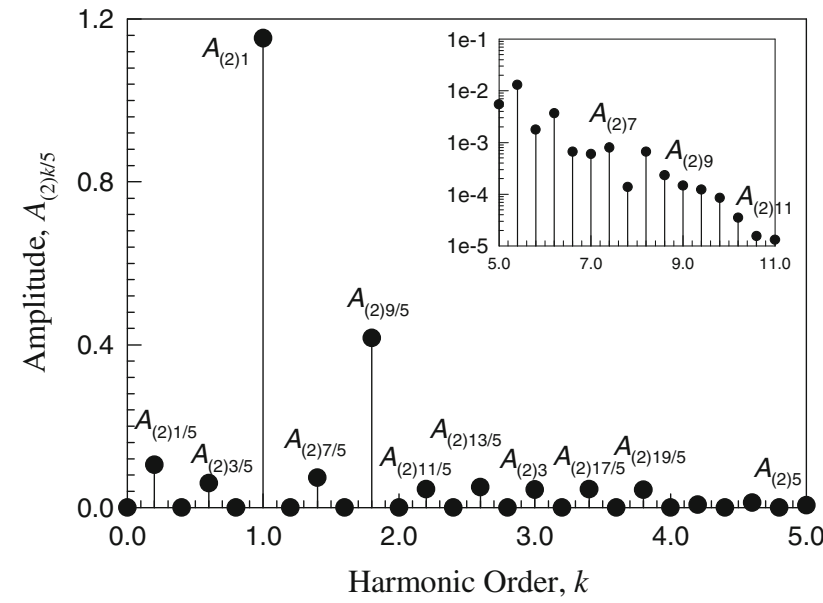

(ix)

Fig. 15 continued

of period-m motions were analyzed through the eigenvalue analysis. Analytical bifurcation trees of period- 1 motions to chaos were presented, and the Hopf bifurcations of periodic motions cause not only the bifurcation tree but quasi-periodic motions. Numerical illustrations of analytical and numerical solutions of periodic solutions were given and displacement orbits of periodic motions in nonlinear rotor systems were employed to show motion complexity, and harmonic amplitude spectrums were used to demonstrate harmonic effects on periodic motions of the nonlinear rotor system.

\section{References}

1. Jeffcott HH (1919) The lateral vibration loaded shaft in the neighborhood of a whirling speed-the effect of want of balance. Philos Mag 37(6):304-314

2. Begg IG (1974) Friction induced rotor whirl-A study in stability. ASME J Eng Ind 96(2):450-453

3. Childs DW (1982) Fractional-frequency rotor motion due to nonsymmetric clearance effects. ASME J Energy Power 104:533-541

4. Choi YS, Noah ST (1987) Nonlinear steady-state responses of a rotor-support system. ASME J Vib Acoust Stress Reliab Des 109:255-261

5. Enrich FF (1988) Higher order subharmonic responses of high speed rotors in bearing clearance. ASME J Vib Acoust Stress Reliab Des 110:9-16
6. Day WB (1987) Asymmetric expansion in nonlinear rotordynamics. Q Appl Math 44:779-792

7. Kim YB, Noah YB (1990) Bifurcation analysis for a modified Jeffcott rotor with bearing clearances. Nonlinear Dyn 1:221-241

8. Choi SK, Noah ST (1994) Mode-locking and chaos in a Jeffcott rotor with bearing clearances. ASME J Appl Mech 61:131-138

9. Kim YB, Noah YB (1996) Quasi-periodic response and stability analysis for a nonlinear Jeffcott rotor. J Sound Vib 190:239-253

10. Chu F, Zhang Z (1998) Bifurcation and chaos in a rub-impact Jeffcott with bearing effects. J Sound Vib 210:1-18

11. Jiang J, Ulbrich $H$ (2001) Stability analysis of sliding whirl in a nonlinear Jeffcott rotor with cross-coupling stiffness coefficients. Nonlinear Dyn 24:269-283

12. Luo ACJ (2012) Continuous dynamical systems. Higher Education Press/L \& H Scientific publishing: Beijing/Glen Carbon.

13. Luo ACJ, Huang J (2012) Analytical dynamics of period-m flows and chaos in nonlinear Systems. Int J Bifurcation Chaos 22(4). Article No. 1250093

14. Luo ACJ, Huang JZ (2012) Analytical routes of period-1 motions to chaos in a periodically forced Duffing oscillator with a twin-well potential. J Appl Nonlinear Dyn 1:73-108

15. Luo ACJ, Huang JZ (2012) Unstable and stable period-m motions in a twin-well potential Duffing oscillator. Discont Nonlinearity Complex 1:113-145

16. Luo ACJ, Huang JZ (2013) Analytical solutions for asymmetric periodic motions to chaos in a hardening Duffing oscillator. Nonlinear Dyn 72:417-438

17. Luo ACJ, Huang JZ (2013) Analytical period-3 motions to chaos in a hardening Duffing oscillator. Nonlinear Dyn. doi:10.1007/ s11071-013-0913-9

18. Luo ACJ, Huang JZ (2013) An analytical prediction of period-1 motions to chaos in a softening Duffing oscillator. International $\mathbf{J}$ Bifurcation Chaos 23. Article No. 1350086

19. Luo ACJ, Huang JZ (2013) Period-3 motions to chaos in a softening Duffing oscillator. Int J Bifurcation Chaos (in press)

20. Luo ACJ, Yu B (2013) Analytical solutions for stable and unstable period-1 motion in a periodically forced oscillator with quadratic nonlinearity. ASME J Vib Acoust 135. Article No. 034503

21. Luo ACJ, Yu B (2013) Complex period-1 motions in a periodically forced, quadratic nonlinear oscillator. J Vib Control. doi:10.1177/ 1077546313490525

22. Luo ACJ, Yu B (2013) Period-m motions and bifurcation trees in a periodically excited, quadratic nonlinear oscillator. Discont Nonlinearity Complex 2(3):263-288

23. Luo ACJ, Laken AB (2013) Analytical solutions for period-m motions in a periodically forced van der Pol oscillator. Int J Dyn Control 1(2):99-115 